\title{
SCALP BlOOD LACTATE FOR THE INTRAPARTUM ASSESSMENT OF FETAL Metabolic Acidosis
}

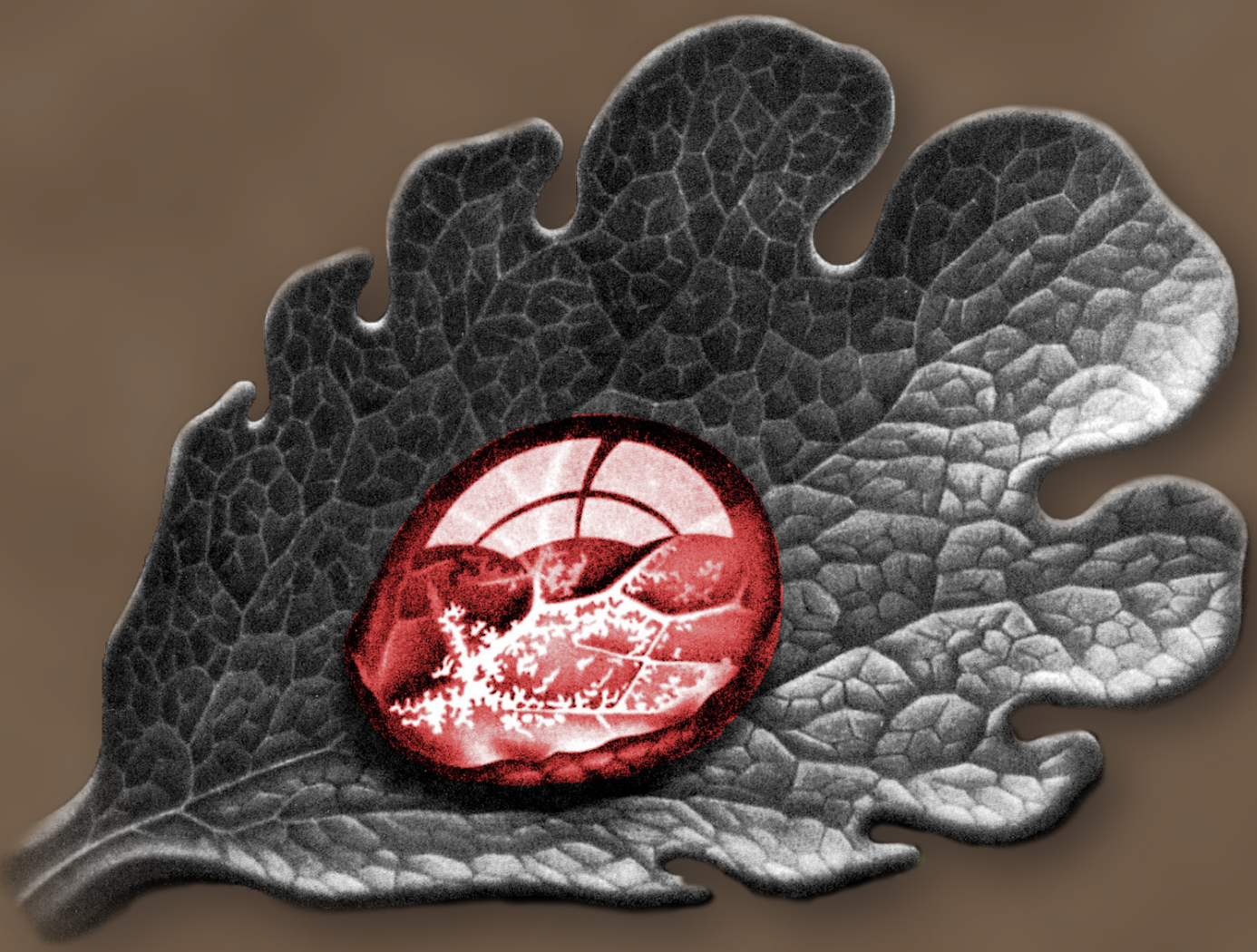

Ayesha Maria Francisca Heinis 



\section{Scalp blood lactate for the intrapartum assessment of fetal metabolic acidosis}




\section{Colofon}

Printing: ProefschriftMaken | Proefschriftmaken.nl

Layout: ProefschriftMaken | Proefschriftmaken.nl

Cover: M.C. Escher's "Dewdrop" (C) 2018 The M.C. Escher Company B.V. - The Netherlands. All rights reserved. www.mcescher.com

\section{Copyright $@$ A.M.F. Heinis, 2018}

ALL RIGHTS RESERVED. Any unauthorized reprint or use of this material is prohibited. No part of this thesis may be reproduced, stored or transmitted in any form or by any means, without written permission of the author or, when appropriate, of the publishers of the publications. 


\title{
Scalp blood lactate for the intrapartum assessment of fetal metabolic acidosis
}

\author{
P R O E F C H R I F T
}

\author{
ter verkrijging van de graad van doctor \\ aan de Radboud Universiteit Nijmegen \\ op gezag van de rector magnificus prof. dr. J.H.J.M. van Krieken, \\ volgens besluit van het college van decanen
}

in het openbaar te verdedigen op woensdag 21 maart 2018 om 14:30 uur precies

door

Ayesha Maria Francisca Heinis

Geboren op 8 maart 1971

te Amstelveen 
Promotor

Prof. dr. F.P.H.A. Vandenbussche

\section{Copromotoren}

Dr. J. van Drongelen

Dr. J. van Dillen

\section{Manuscriptcommissie}

Prof. dr. M.T.E. Hopman

Prof. dr. E.A.P. Steegers (Erasmus MC)

Dr. N. Wiberg (Skånes universitetssjukhus Zweden) 
Voor mijn moeder 



\section{Table of contents}

Chapter 1 General introduction and outline of the thesis 9

Chapter 2 Inter- and intra-observer agreement of non-reassuring 21 cardiotocography analysis and subsequent clinical management

Chapter 3 Scalp blood lactate for intra-partum assessment of fetal metabolic acidosis

Chapter 4 Comparison of two point-of-care testing devices for fetal lactate during labor

Chapter 5 Clinical evaluation of Statstrip ${ }^{\oplus}$ Lactate for use in fetal scalp blood sampling

Chapter 6 Two-year follow up of an expanded fetal monitoring protocol aimed at increasing the success rate of fetal blood sampling

Chapter 7 General discussion and conclusions

Chapter 8 Summary \& samenvatting

Addendum Dankwoord 


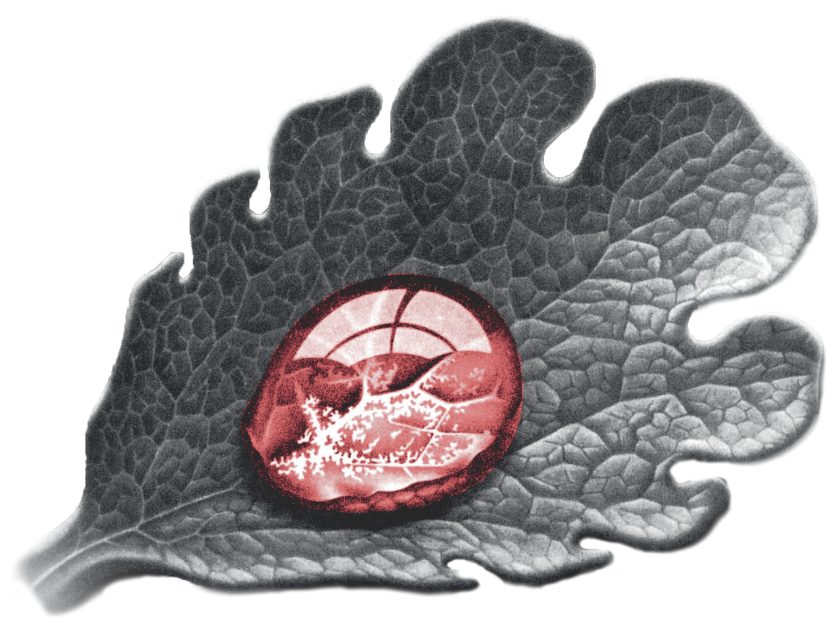




\section{Chapter 1}

GENERAL INTRODUCTION AND OUTLINE OF THE THESIS 



\section{General introduction}

Being born is a great challenge. In utero, the fetus is totally depended on the mother through the placenta and umbilical cord, and must establish itself as a dependent air breathing individual. This transition can be complicated by hypoxia and acidosis. This makes intrapartum surveillance a prime task for midwives and obstetricians. The goal is to identify the fetus that will benefit from timely intervention before substantial damage has occurred.

For this purpose, cardiotocography (CTG) - a simultaneous recording of the fetal heart rate and the contractions of the uterus -, was introduced into obstetric practice during the 1970s and 1980s and is still a key component of modern obstetrics. The introduction was made on the assumption that CTG would help to reduce cerebral palsy caused by intra partum hypoxia/acidosis. Forty years have since passed and there has not been any evidence to show an improvement in cerebral palsy (Figure 1). What has changed dramatically though, is the cesarean section (CS) rate, which has risen four- to fivefold. The sad conclusion is that this has cost us more in terms of maternal morbidity and mortality than it has benefited babies (1).

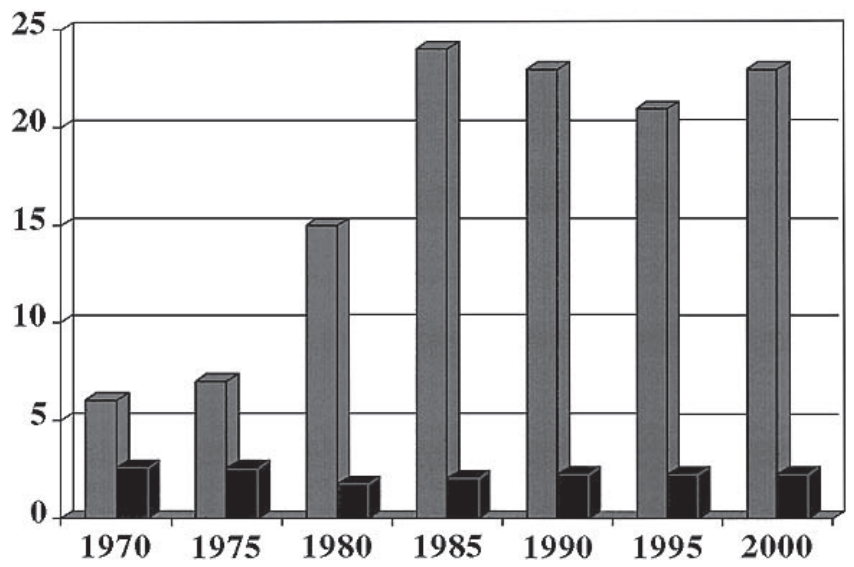

Figure 1. Cerebral palsy prevalence (black bars) and the cesarean section rate (dark gray bars) in developed countries. From: Clark SL, Hankins GD. Temporal and demographic trends in cerebral palsy--fact and fiction. American Journal of Obstetrics and Gynecology. 2003. 
The main reason for this is the high inter-and intra observer variation of CTG; whereas a normal CTG tracing almost entirelyexcludes hypoxia/acidosis, a non-reassuring or abnormal tracing has a high false positive rate of at least $50 \%$ for this outcome $(2,3)$. Lack of universally accepted guidelines and poor inter observer agreement on tracing interpretation have probably contributed to the limited effectiveness of CTG monitoring. The International Federation of Gynecology and Obstetrics (FIGO) published its first guidelines on CTG interpretation in 1987 and established the only international consensus guideline available to date. Since then, several national scientific organizations have published intra partum fetal monitoring guidelines including; the American College of Obstetricians and Gynecologists (ACOG), the National Institute of Child Health and Human Development (NICHHD), the Royal College of Obstetricians and Gynaecologists (RCOG), and the National Institute of Clinical Excellence (NICE). These guidelines differ substantially, not only in the definition of individual CTG features but also in the criteria used for overall tracing classification (4). These differences have a profound effect on the inter-observer agreement and reliability, on the way clinicians interpret CTG tracings, as well as on the predictive value of CTG classifications for fetal acidemia (5).

Consensus guidelines are usually developed by experts, but the majority of healthcare professionals that need to apply them have other areas of main professional focus. It is therefore likely that many of the concepts contained in guidelines are only partly assimilated and are prone to be easily forgotten. In order to increase assimilation and the retention of knowledge it is better to keep guidelines as simple as possible and to establish a clear association between the different tracing classifications and subsequent clinical management, for example: normal tracings - no actions required; suspicious tracings - continue monitoring, perform additional testing, or minor interventions (such as reducing maternal temperature); pathological tracings - immediate intervention to revert the cause of fetal hypoxia or rapid delivery if reversal is not foreseen.

In the Netherlands, the national guideline on fetal monitoring by the Dutch Society of Obstetricians and Gynecologists (NVOG) provides such an association between CTG classification and subsequent clinical management (6). Besides this national guideline, various local protocols exist. The NVOG guideline is frequently used (58\%), sometimes in combination with a local guideline (26\%). In 36\% of hospitals, only local protocols are used (7). It is not known whether and how the variation in guidelines influences the variation in clinical practice with regards to the diagnosis and management of fetal distress during labor. What is known is that fetal monitoring with CTG only results in variable, but inappropriately high operative delivery rates. 
Over the last four decades, many methods have been proposed to reduce this high rate with the use of supplementary tests to confirm or exclude the diagnosis of hypoxia/ acidosis in case of CTG abnormalities. The two best known and accepted ones are fetal blood sampling (FBS) and ST analysis of the fetal electrocardiogram (STAN).

The technique of FBS was first described by Erich Saling in Berlin in 1962 (Figure 2) (8). This extract from his original paper in 1966 outlines the FBS technique; "The presenting part of the foetus is visualized, either with the help of a conical endoscope or, if the cervix is sufficiently dilated, with a speculum. After making the skin hyperaemic by freezing with ethylchloride spray, it is smeared with a layer of paraffin oil so that the blood will collect in a drop. A stab incision is made. The blood that collects is sucked into a heparinized capillary tube, and its $\mathrm{pH}$ measured. The Astrup equipment is suitable for pH measurement, and allows also the Pco2, bicarbonate, and base excess to be measured. An acidosis can thus be characterized as either metabolic (lowered bicarbonate and baseexcess), or respiratory (raised Pco2)".

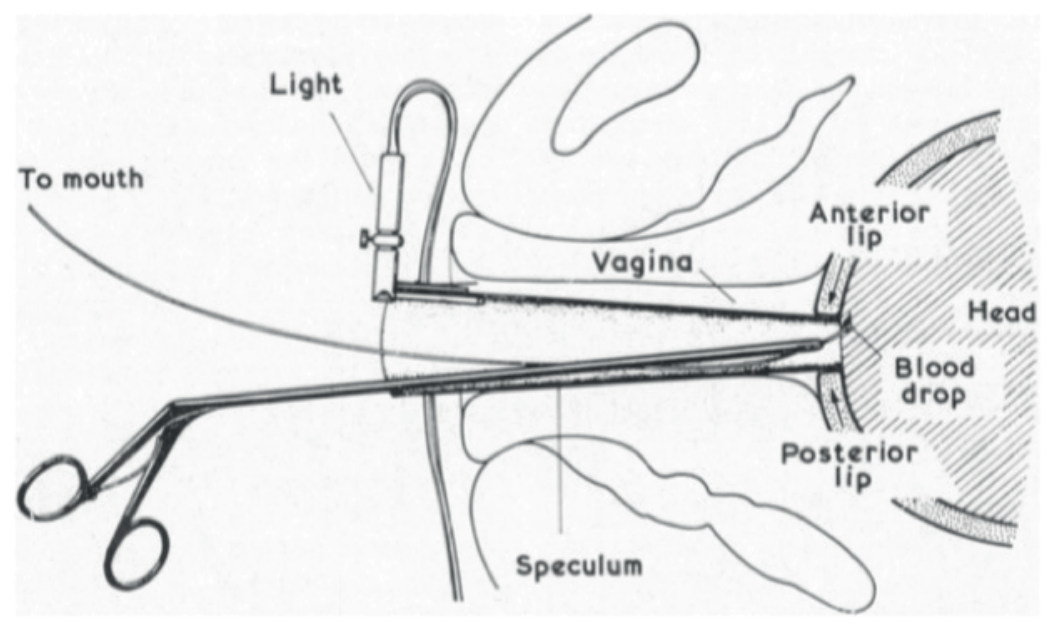

Figure 2. Foetal blood sampling. The presenting part has been pricked, and a drop of blood is about to be sucked into a heparinized tube, held in special forceps.

Saling chose a $\mathrm{pH}<7.20$ as a cut-off value to recommend intervention. Based on a series of 306 fetuses, which were all born in a vigorous state, $\mathrm{pH}$ normal ranges were calculated by adding and subtracting two standard deviations from the mean (Figure 3) (8). Almost 20 years later, Saling reported on the number of fetal deaths over a 30-year period among 50000 deliveries during the change in fetal monitoring 
from auscultation alone (1955-1960) to auscultation in combination with FBS (1961-1967) to CTG and FBS (1968-1979). Intra partum deaths fell in these three time periods from 0.8 to $0.32 \%$, and then to $0.15 \%$. He credited FBS as the main reason for this decline (9). FBS was introduced as an additional clinical test in many obstetric units during the 1970s and 1980s and has remained in daily use.

\begin{tabular}{|c|c|c|c|c|c|c|c|}
\hline & $\mathrm{E}_{1-3}$ & $E_{6-8}$ & $E_{10-12}$ & BB & BA & NV & NA \\
\hline $\begin{array}{l}\text { Obere Grenze des Normal- } \\
\text { bereidhes. }\end{array}$ & 7,40 & 7,46 & 7,42 & 7,40 & 7,42 & 7,45 & 7,40 \\
\hline Mittelwerte . . . . & 7,31 & 7,33 & 7,31 & 7,30 & 7,28 & 7,30 & 7,25 \\
\hline $\begin{array}{l}\text { Untere Grenze des Normal- } \\
\text { bereiches. }\end{array}$ & 7,23 & 7,19 & 7,21 & 7,21 & 7,14 & 7,15 & 7,09 \\
\hline
\end{tabular}

Figure 3. Cut-off values for $\mathrm{pH}$ in the different stages of labor and post-partum in the umbilical vessels from the original paper by Saling in 1962.

There are some practical problems surrounding FBS. The analysis of $\mathrm{pH}$ is complicated; a relatively large amount of blood (30-50 $\mu \mathrm{l})$ is needed, and sampling failure rates are high (up to $20 \%)(10,11)$. In addition, $\mathrm{pH}$ does not discriminate between respiratory and metabolic acidemia, the latter being associated with poor neonatal outcome (12). Saling and others have therefore suggested to add base deficit (BD) to FBS, as occurs in testing umbilical cord blood. However, BD is a calculated value from $\mathrm{pH}$ and $\mathrm{pCO}$, and algorithms for its calculation are intended for adults and differ between blood gas analyzers. Moreover, no cut-off values for intervention are available. This makes the role of $\mathrm{BD}$ in FBS questionable. In contrast, lactate is the measured end product of anaerobe glycolysis and therefore a good marker of metabolic acidosis. Point of Care (POC) lactate testing devices need only a tiny amount of blood, deliver fast results, and have a high success rate, which is very convenient in FBS (13-15). In a large randomized controlled trial of 3000 women, lactate was compared with $\mathrm{pH}$ in FBS, no significant differences were found in terms of mode of delivery or neonatal outcome (16). Therefore, POC testing of blood lactate can be used as an alternative for $\mathrm{pH}$ analysis in FBS. There is no established "gold standard" for lactate determinations and therefore the calibration alignment of POC lactate devices can differ. As such comparisons between different devices have usually shown excellent correlations but often with differences in absolute values. Therefore, reported lactate concentrations should be regarded as a figure on a 
relative scale rather than an exact measurement of a concentration. For POC lactate measurements, several devices are available. Lactate Pro ${ }^{\text {тм }}$ (LP) (Arkray, Kyoto, Japan) is an electrochemical test strip device which requires a blood sample of $5 \mu \mathrm{L}$, with result available after 60 seconds at a low failure rate (1-2\%). Based on observational data, cut-off values for lactate in FBS measured with LP were suggested (17). These cut-off values were tested in the above mentioned RCT, where lactate was found to be at least as good as $\mathrm{pH}$ in the management of non-reassuring CTG, with significantly less sampling/analysis failure rates (14). Unfortunately, the production of the LP meter is discontinued. Another POC lactate device is Statstrip Lactate (SSL) (Nova Biomedical). This device utilizes $0.7 \mu \mathrm{L}$ of blood and results are available in 13 sec.

Another example of an adjunctive test to CTG is STAN. This technique detects changes in the ST segment of the fetal ECG (ST events) during labor, which are related to fetal hypoxia. The occurrence of these ST events is interpreted together with CTG, according to the STAN clinical guidelines. Meta-analysis comparing STAN to CTG with or without FBS has confirmed a lower rate of metabolic acidosis (RR 0.64, 95\% CI 0.46-0.88), a reduction in the rate of FBS (RR 0.59, 95\% CI $0.45-0.79$ ) and a reduction in the overall rate of assisted vaginal delivery (RR 0.92, 95\% CI 0.86-0.99). There was no reduction in the rate of operative delivery for fetal distress, perinatal death, seizures and encephalopathy (18). Two trials have shown that STAN is cost-effective when compared to CTG used in conjunction with FBS $(19,20)$. It is unclear if the use of STAN makes FBS redundant or not. An important issue in intrapartum fetal monitoring, is avoiding the occurrence of false-negative test results leading to the birth of an infant with adverse outcome. There have been reports of false negative results associated with the use of the STAN technique (2123). Based on these reports, the existing STAN clinical guidelines were updated in January 2007, to include recommendations for situations in which additional fetal information is required, such as performing an FBS test (24).

In the Netherlands, a large practice variation exists in fetal surveillance during labor. Although all Dutch labor wards use CTG, in 23\% ( $\mathrm{N}=20)$ of the hospitals STAN is used to monitor fetal well-being. In all but one hospital (98\%), FBS facilities are available, but the frequency of FBS use varies between $3-15 \%$ of deliveries $(7,25)$. It is suggested that this is mainly due to the variation in the indication to perform FBS. Evidence based guidelines on when to perform FBS are lacking. Furthermore, there is ongoing discussion on whether to use $\mathrm{pH}$ or lactate as a marker in FBS. As there is still uncertainty about the scalp lactate level at which to initiate intervention during labor most Dutch hospitals continue to test $\mathrm{pH}$ in FBS. 
The ideal method for intra partum fetal monitoring would be simple, noninvasive, and applicable to all women in labor, irrespective of gestational age. Furthermore, the method should ideally satisfy three criteria. Firstly, the method should give an early warning (when the baby is only pre-acidemic), to provide time for the clinician to deliver the baby without putting the mother at unnecessary risk. Secondly, system feedback should be clear ("do we have a problem? yes or no") and easy to follow guidelines should be available. Thirdly, the test should be able to identify the nonhypoxic fetuses so unnecessary intervention can be avoided. Neither FBS, nor STAN fulfill all of these criteria, but their integrated use could be the way to move forward.

In this thesis, we wish to elaborate further on these challenges with the following aims:

- To study the inter- and intra-observer agreement of non-reassuring CTG patterns and subsequent clinical management (Chapter 2).

- To investigate to what extent the fetal scalp blood lactate concentration during labor correlates with fetal scalp pH, base deficit, and metabolic acidosis at birth, and to identify which lactate cut-off values can serve as indicators for either reassurance or immediate intervention (Chapter 3).

- To study the performance of two POC lactate meters when used for scalp blood lactate analysis during labor (Chapter 4).

- To evaluate the Statstrip Lactate for its use in FBS in the clinical setting (Chapter 5).

- To investigate the effects of the implementation of an expanded fetal monitoring protocol consisting of STAN and FBS with combined lactate and $\mathrm{pH}$ testing, on FBS success, delivery intervention rates and perinatal outcome (Chapter 6). 


\section{References}

(1) Clark SL, Hankins GD. Temporal and demographic trends in cerebral palsy--fact and fiction. American journal of obstetrics and gynecology. 2003;188(3):628-33.

(2) Lotgering FK, Wallenburg HC, Schouten HJ. Inter-observer and intra-observer variation in the assessment of antepartum cardiotocograms. American journal of obstetrics and gynecology. 1982;144(6):701-5.

(3) Larma JD, Silva AM, Holcroft CJ, Thompson RE, Donohue PK, Graham EM. Intrapartum electronic fetal heart rate monitoring and the identification of metabolic acidosis and hypoxic-ischemic encephalopathy. American journal of obstetrics and gynecology. 2007;197(3):301 e1-8.

(4) Ayres-de-Campos D, Bernardes J, Subcommittee F. Twenty-five years after the FIGO guidelines for the use of fetal monitoring: time for a simplified approach? Int J Gynaecol Obstet. 2010;110(1):1-6.

(5) Santo S, Ayres-de-Campos D, Costa-Santos C, Schnettler W, Ugwumadu A, Da Graca LM, et al. Agreement and accuracy using the FIGO, ACOG and NICE cardiotocography interpretation guidelines. Acta Obstet Gynecol Scand. 2017;96(2):166-75.

(6) Nederlandse Vereniging voor Obstetrie en Gynaecologie. Richtlijn Foetale Bewaking [Fetal monitoring during labor]. Available from: www.nvog.nl; June 2017 [Dutch].

(7) Bullens LM, Moors S, van Runnard Heimel PJ, van der Hout-van der Jagt MB, Oei SG. Practice variation in the management of intrapartum fetal distress in The Netherlands and the Western world. Eur J Obstet Gynecol Reprod Biol. 2016;205:48-53.

(8) Saling E. [A new method for examination of the child during labor. Introduction, technique and principles] (in German). Arch. Gynakol. 1962;197:108-22.

(9) Salin E. Fetal scalp blood analysis; current practice. J.Perinat Med. 1981;9:165-77.

(10) Westgren M, Kruger K, Ek S, Grunevald C, Kublickas M, Naka K, et al. Lactate compared with $\mathrm{pH}$ analysis at fetal scalp blood sampling: a prospective randomised study. Br J Obstet Gynaecol. 1998;105(1):29-33.

(11) Tuffnell D, Haw WL, Wilkinson K. How long does a fetal scalp blood sample take? BJOG. 2006;113(3):332-4.

(12) Low JA, Panagiotopoulos C, Derrick EJ. Newborn complications after intrapartum asphyxia with metabolic acidosis in the term fetus. American journal of obstetrics and gynecology. 1994;170(4):1081-7.

(13) Nordstrom L. Fetal scalp and cord blood lactate. Best Pract Res Clin Obstet Gynaecol. 2004;18(3):467-76.

(14) Nordstrom L, Persson B, Shimojo N, Westgren M. Fetal scalp and umbilical artery blood lactate measured with a new test strip method. Br J Obstet Gynaecol. 1992;99(4):307-9.

(15) Ramanah R, Martin A, Clement MC, Maillet R, Riethmuller D. Fetal scalp lactate microsampling for non-reassuring fetal status during labor: a prospective observational study. Fetal Diagn Ther. 2010;27(1):14-9. 
(16) Wiberg-Itzel E, Lipponer C, Norman M, Herbst A, Prebensen D, Hansson A, et al. Determination of $\mathrm{pH}$ or lactate in fetal scalp blood in management of intrapartum fetal distress: randomised controlled multicentre trial. BMJ. 2008;336(7656):1284-7.

(17) Kruger K, Hallberg B, Blennow M, Kublickas M, Westgren M. Predictive value of fetal scalp blood lactate concentration and $\mathrm{pH}$ as markers of neurologic disability. American journal of obstetrics and gynecology. 1999;181(5 Pt 1):1072-8.

(18) Blix E, Brurberg KG, Reierth E, Reinar LM, Oian P. ST waveform analysis versus cardiotocography alone for intrapartum fetal monitoring: a systematic review and meta-analysis of randomized trials. Acta Obstet Gynecol Scand. 2016;95(1):16-27.

(19) Heintz E, Brodtkorb TH, Nelson N, Levin LA. The long-term cost-effectiveness of fetal monitoring during labor: a comparison of cardiotocography complemented with ST analysis versus cardiotocography alone. BJOG. 2008;115(13):1676-87.

(20) Vijgen SM, Westerhuis ME, Opmeer BC, Visser GH, Moons KG, Porath MM, et al. Cost-effectiveness of cardiotocography plus ST analysis of the fetal electrocardiogram compared with cardiotocography only. Acta Obstet Gynecol Scand. 2011;90(7):772-8.

(21) Westerhuis ME, Kwee A, van Ginkel AA, Drogtrop AP, Gyselaers WJ, Visser GH. Limitations of ST analysis in clinical practice: three cases of intrapartum metabolic acidosis. BJOG. 2007;114(10):1194-201.

(22) Ingemarsson I, Westgren M. ST analysis. BJOG. 2007;114(11):1445.

(23) Doria V, Papageorghiou AT, Gustafsson A, Ugwumadu A, Farrer K, Arulkumaran S. Review of the first 1502 cases of ECG-ST waveform analysis during labor in a teaching hospital. BJOG. 2007;114(10):1202-7.

(24) Amer-Wahlin I, Arulkumaran S, Hagberg H, Marsal K, Visser GH. Fetal electrocardiogram: ST waveform analysis in intrapartum surveillance. BJOG. 2007;114(10):1191-3.

(25) Westerhuis ME, Strasser SM, Moons KG, Mol BW, Visser GH, Kwee A. [Intrapartum foetal monitoring: from stethoscope to ST analysis of the ECG]. Ned Tijdschr Geneeskd. 2009;153:B259. 



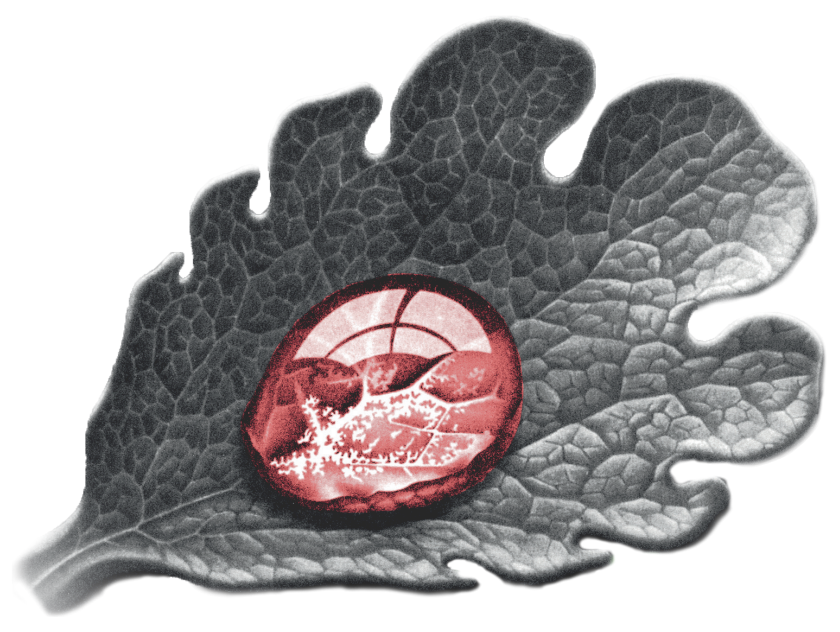




\section{Chapter 2}

INTER- AND INTRA-OBSERVER AGREEMENT OF NON-REASSURING CARDIOTOCOGRAPHY ANALYSIS AND SUBSEQUENT CLINICAL MANAGEMENT

Sarah Rhöse, Ayesha MF Heinis, Frank Vandenbussche, Joris van Drongelen, Jeroen van Dillen

Acta Obstet Gynecol Scand. 2014 Jun;93(6):596-602. 


\section{Abstract}

Objective: To quantify inter- and intra-observer agreement of non-reassuring intrapartum cardiotocography (CTG) patterns and subsequent clinical management.

Design: Methodological study.

Setting: University Medical Center.

Population: CTG patterns of 97 women beyond 37 weeks of gestation with a singleton fetus in vertex position in first stage of labor in whom fetal blood sampling (FBS) had been performed.

Methods: Nine observers assessed CTG patterns, which were formerly clinically classified as non-reassuring and indicative for FBS, according to the guidelines of the International Federation of Gynecology and Obstetrics modified for ST analysis. They also proposed clinical management strategies without and with insight into clinical parameters. Weighted kappa values $\left(\mathrm{K}_{\mathrm{w}}\right)$ and proportions of agreement $\left(\mathrm{P}_{\mathrm{a}}\right)$ were calculated.

Main Outcome Measures: Agreement on CTG classification and clinical management.

Results: Inter-observer agreement on CTG classification and on clinical management were poor for most observer categories $\left(\mathrm{K}_{\mathrm{w}}\right.$ range $0.31-0.50$ and $0.20-0.45$, respectively). Observers agreed best on abnormal CTG patterns $\left(\mathrm{P}_{\mathrm{a}}\right.$ range $\left.0.28-0.36\right)$ and on the clinical management option 'continue monitoring' ( $\mathrm{P}_{\mathrm{a}}$ range $\left.0.32-0.40\right)$. Intra-observer agreement was fair to good for most observers $\left(\mathrm{K}_{\mathrm{w}} 0.33-0.70\right)$. Insight into clinical parameters resulted in similar inter- and intra-observer agreement.

Conclusions: There was poor inter-observer agreement and fair to good intraobserver agreement on classification and clinical management of intrapartum CTG patterns, which had been classified as non-reassuring and indicative for FBS during birth. 


\section{Introduction}

CTG is a technique used to monitor intrapartum fetal condition. It is a continuous simultaneous record of the fetal heart rate and of the presence of uterine activity (1). To classify a CTG pattern several classification systems exist, of which the FIGO/ STAN guidelines have probably reached broadest consensus (2). One of the main disadvantages of the CTG is that there is substantial variation in interpreting CTG patterns. Previous research has shown that inter- and intra-observer agreement of CTG classification is often poor (3-6). Also, the specificity of a CTG is low, i.e. many false-positive test results for poor neonatal outcome (7). As a consequence, unnecessary obstetric interventions for suspected fetal distress are performed (1, 7-9). In an attempt to reduce these unnecessary interventions, FBS can be used as a diagnostic test to gain additional information on fetal condition (10). Nevertheless, FBS is an invasive, cumbersome procedure with failure rates up to $20 \%$ (8), and it is only assumed that FBS reduces the cesarean section rate. Therefore, its use should be balanced in an effort to keep both cesarean section rate and FBS rate at a reasonable level.

Various observations suggest considerable variation in the indication to perform FBS. Firstly, FBS use varies between countries, hospitals and care workers. In the Netherlands FBS is performed in 3 to $15 \%$ of deliveries (11), while these percentages are different in other countries (12-14). Secondly, evidence based guidelines on when to perform FBS are lacking. $(15,16)$ Also the impression exists that FBS is performed on more liberal indications than described in these guidelines, i.e. for reassurance on fetal condition before active pushing is started. Hence, there is significant variation in the use of FBS, which could be explained by variation in the indication to perform FBS.

The objectives of this study were to quantify variation (i.e. inter- and intra-observer agreement) in (i) the classification of intrapartum CTG patterns prior to FBS, according to the FIGO/STAN guidelines (17), and (ii) management based on this classification. The impact of insight into clinical parameters and also of the observers' level of experience was analyzed. In addition, the relation between fetal scalp pH and CTG category was evaluated. 


\section{Material and methods}

We performed a methodological study at the Radboud University Medical Center, which is a tertiary referral hospital with approximately 1500 deliveries a year. In the Netherlands, independent primary care midwives provide care to low risk women. If complications or risk factors occur during pregnancy, labor, or after birth, women are referred to secondary or tertiary care. After referral, care may be provided by clinical midwives or obstetric registrars but always under final responsibility of an obstetrician. Fetal condition during labor is monitored with continuous CTG and FBS is performed in $11 \%$ of deliveries (18). The Research Ethics Committee at the Radboud University Nijmegen Medical Center approved the study (2012/080).

We selected all women who delivered between February 2010 and March 2011 and in whom FBS was performed based on a non-reassuring CTG during the first stage of labor. Women were included if they complied to the following inclusion criteria: singleton fetus in vertex position, gestational age of 37 weeks or over, and optimal CTG registration 60 minutes prior to FBS.

Nine observers were asked to participate in the study. They were divided into three groups according to their specialty level and level of experience in obstetrics. Group A contained four obstetricians with at least one year of experience as obstetrician. Group B contained three registrars gynecology in their second or third educational year (full educational period is six years with obstetrics incorporated in each year). Group C contained two clinical midwives with two to three years of

clinical experience. All observers use fetal monitoring with CTG regularly in clinical practice. They received training as standard part of their education. Knowledge about CTG interpretation is exchanged in the daily morning protocol were CTG patterns are discussed with attending midwives, registrars and obstetricians.

For each case, we extracted CTG patterns from the obstetric database of the Radboud University Medical Center. We identified the 60-minute CTG pattern prior to FBS. If fetal blood was sampled more than once, the CTG pattern prior to the first sample was used. We also extracted clinical parameters, including general information (maternal age, obstetric history and risk factors in the current pregnancy), parameters that influence progression of labor (parity, cervical dilatation, augmentation of labor) and parameters that influence fetal condition (gestational age, the presence of meconium stained amniotic fluid, maternal body temperature and estimated fetal weight). 
The nine observers were asked to assess the CTG patterns twice with an interval of at least one month: T0 and T1. To assess the CTG patterns, a web-based tool was developed which provided the observers with CTG patterns and corresponding clinical parameters. This web-based tool did not allow observers to change previous answers and presented the cases in random order at T0 and T1. The assessment procedure went as follows. First, the observers were shown the anonymized CTG pattern (paper speed $2 \mathrm{~cm} / \mathrm{min}$, that is commonly used in the Netherlands) and were asked to classify it according to the FIGO/STAN guidelines into one of four categories: normal, intermediary, abnormal or pre-terminal. At this time observers were provided with the information that it concerned a CTG pattern from the dilatation phase, of a singleton fetus in vertex position in whom FBS had been performed during labor. Secondly, observers were asked to choose a clinical management option: (i) continue monitoring with the availability of measures to solve the causes of fetal distress by conservative management (for example treating hypotension, discontinuing oxytocin infusion, changing maternal position), (ii) perform FBS or (iii) immediate delivery (within 15 minutes). Thirdly, observers were provided with the corresponding clinical parameters and were then asked again to choose a clinical management option.

\section{Statistical analyses}

CTG classification and clinical management strategy by different observers were used to calculate inter-observer agreement. Intra-observer agreement was calculated using observations of the same observer at T0 and T1. SPSS (SPSS for Windows, Rel. 18.0.2. 2010. Chicago: SPSS Inc.) and SAS (SAS Institute Inc. 2010. SAS/ GRAPH $^{\odot}$ 9.2 Reference, Second Edition. Cary, NC: SAS Institute Inc.) were used for the data analysis.

For the categorical, ordinal variables 'CTG classification' and 'clinical management' $\mathrm{Kw}$ (weighted kappa) was calculated. $\mathrm{Pa}$ (proportions of agreement) were calculated for each individual CTG category and clinical management option (19). Weighted kappa values above 0.75 were considered as excellent agreement, between 0.40 and 0.75 as fair to good and below 0.40 as poor agreement.

Both $\mathrm{Kw}$ and $\mathrm{Pa}$ describe the amount of agreement, but in a different way. $\mathrm{Kw}$ measures agreement beyond agreement expected by chance (20). It measures the degree of association between two variables, but not their true agreement (19). Pa shows the proportion of cases on which observers agree (20). A limitation of $\mathrm{Pa}$ is that it does not take into account agreement expected by chance. 
Prior to this study, we performed power analysis. A sample size estimation for analyzing agreement (kappa) between two observers was made with $\alpha=0.05$ and $\beta$ $=0.2$. To investigate the null hypothesis $\mathrm{H} 0: \mathrm{p} 0=0.4$ and the alternative hypothesis H1: p1 $=0.6$, a sample size of 90-100 CTG patterns was estimated $(20,21)$.

For correlation analysis of CTG classification and scalp $\mathrm{pH}$, we selected those cases in which consensus on CTG classification was reached. We defined consensus as CTG category inter-observer agreement of more than $75 \%$ of observers $(\mathrm{n} \geq 7)$. Scalp pH was considered non-reassuring if $\mathrm{pH}$ was below or equal to 7.25 and acidemic if $\mathrm{pH}$ was below 7.20. For each CTG category the proportion of non-reassuring scalp $\mathrm{pH}$ was calculated. 


\section{Results}

During the study period 1755 women delivered at the Radboud University Medical Center. Ninety-seven women met the inclusion criteria. Table 1 summarizes the characteristics of the mother and baby pairs. In 15 cases scalp pH was non-reassuring, in one case scalp pH was acidotic and in 14 cases scalp pH analysis had failed.

Table 1. Characteristics of 97 mother and baby pairs

\begin{tabular}{|c|c|c|}
\hline \multirow{2}{*}{$\begin{array}{l}\text { Characteristic } \\
\text { Maternal age in (years) }\end{array}$} & \multicolumn{2}{|c|}{ Number (\%) or median (range) } \\
\hline & 31 & $(19-43)$ \\
\hline Gestational age (days) & 281 & $(263-295)$ \\
\hline Nulliparous women & 78 & $(80)$ \\
\hline \multicolumn{3}{|l|}{ Estimated fetal weight } \\
\hline $\mathrm{SGA}^{1}$ & 8 & $(8)$ \\
\hline $\mathrm{LGA}^{2}$ & 5 & $(5)$ \\
\hline Cervical dilatation at time of FBS $(\mathrm{cm})$ & 7 & $(3-10)$ \\
\hline Maternal fever ${ }^{3}$ & 16 & $(16)$ \\
\hline Augmentation of labor & 71 & $(73)$ \\
\hline Meconium stained amniotic fluid & 25 & $(26)$ \\
\hline FBS $\mathrm{pH} \leq 7.25^{4}$ & 15 & $(15)$ \\
\hline Birthweight (grams) & 3440 & $(2405-4618)$ \\
\hline $\mathrm{SGA}^{5}$ & 11 & $(11)$ \\
\hline LGA $^{6}$ & 9 & (9) \\
\hline
\end{tabular}

${ }^{1}$ Small for Gestational Age (SGA): estimated fetal weight below the $10^{\text {th }}$ percentile of the Verburg growth curve (25) ${ }^{2}$ Large for Gestational Age (LGA): estimated fetal weight above the $90^{\text {th }}$ percentile of the Verburg growth curve (25) ${ }^{3}$ Maternal temperature $\geq 37.8{ }^{0}{ }_{4}^{4}$ Fetal blood sampling (FBS) ${ }^{5}$ Small for Gestational Age (SGA), i.e. below the $10^{\text {th }}$ percentile of the National Perinatal registry curves (26) ${ }^{6}$ Large for Gestational Age (LGA) i.e. above the $90^{\text {th }}$ percentile of the National Perinatal registry curves (26).

All observers assessed 97 CTG patterns at T0 and T1. Mean assessment time of all CTG patterns at T0 was 78 minutes, ranging from 46 to 121 minutes. At T1 mean assessment time was 58 minutes, ranging from 29 to 97 minutes. Most CTG patterns were classified intermediary or abnormal (Figure 1). The clinical management strategy 'FBS' was chosen in $23 \%$ of CTG patterns classified as intermediary and in $72 \%$ of CTG patterns classified as abnormal (Figure 1). 


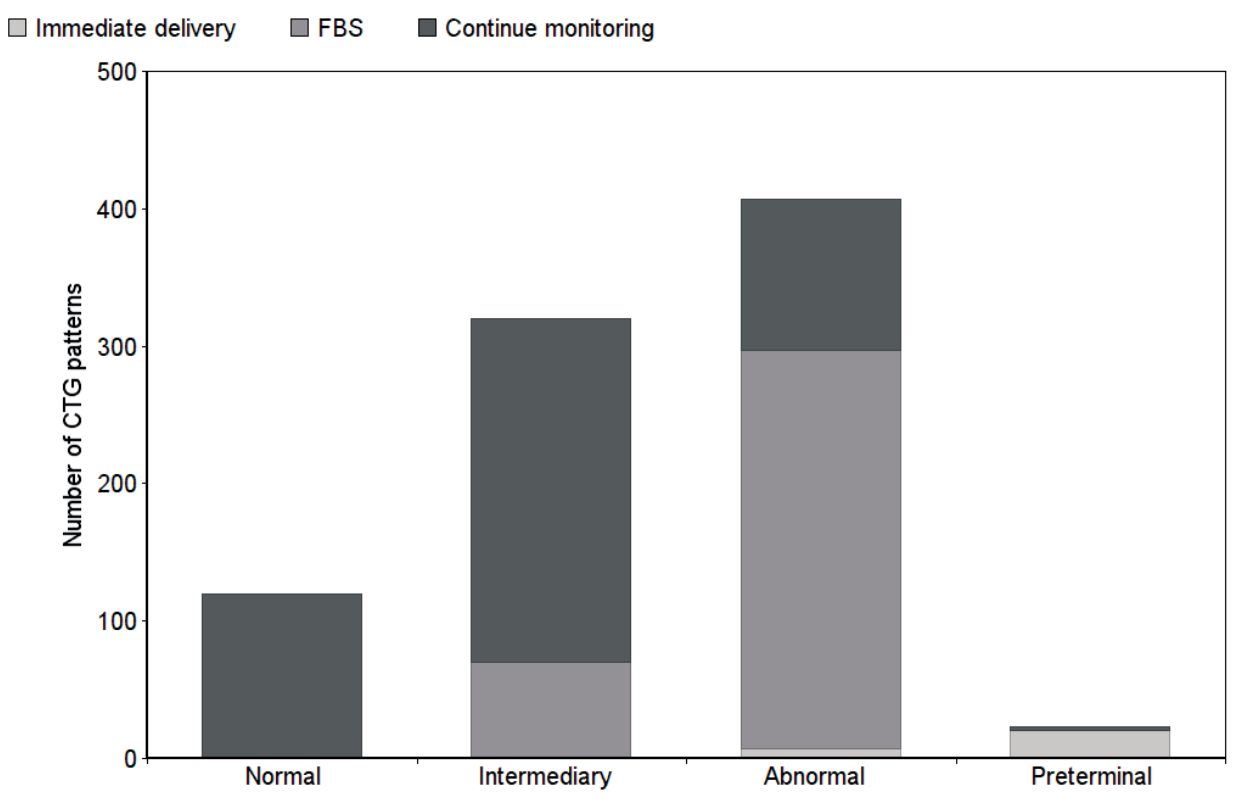

Figure 1. Clinical management strategy per cardiotocography (CTG) category at $T_{0}$ (total number of CTG patterns was 873 as nine observers each assessed 97 CTG patterns). FBS, fetal blood sampling.

At T0, inter-observer agreement on classification of CTG patterns was poor within and between most observer categories, except for clinical midwives, whose agreement was fair to good (Table 2). Agreement was best for abnormal CTG patterns (Pa all observers 0.31). Agreement on clinical management was poor within and between most observer categories (Table 3). Registrars and clinical midwives agreed moderately. Observers agreed best on the clinical management option 'continue monitoring'. 

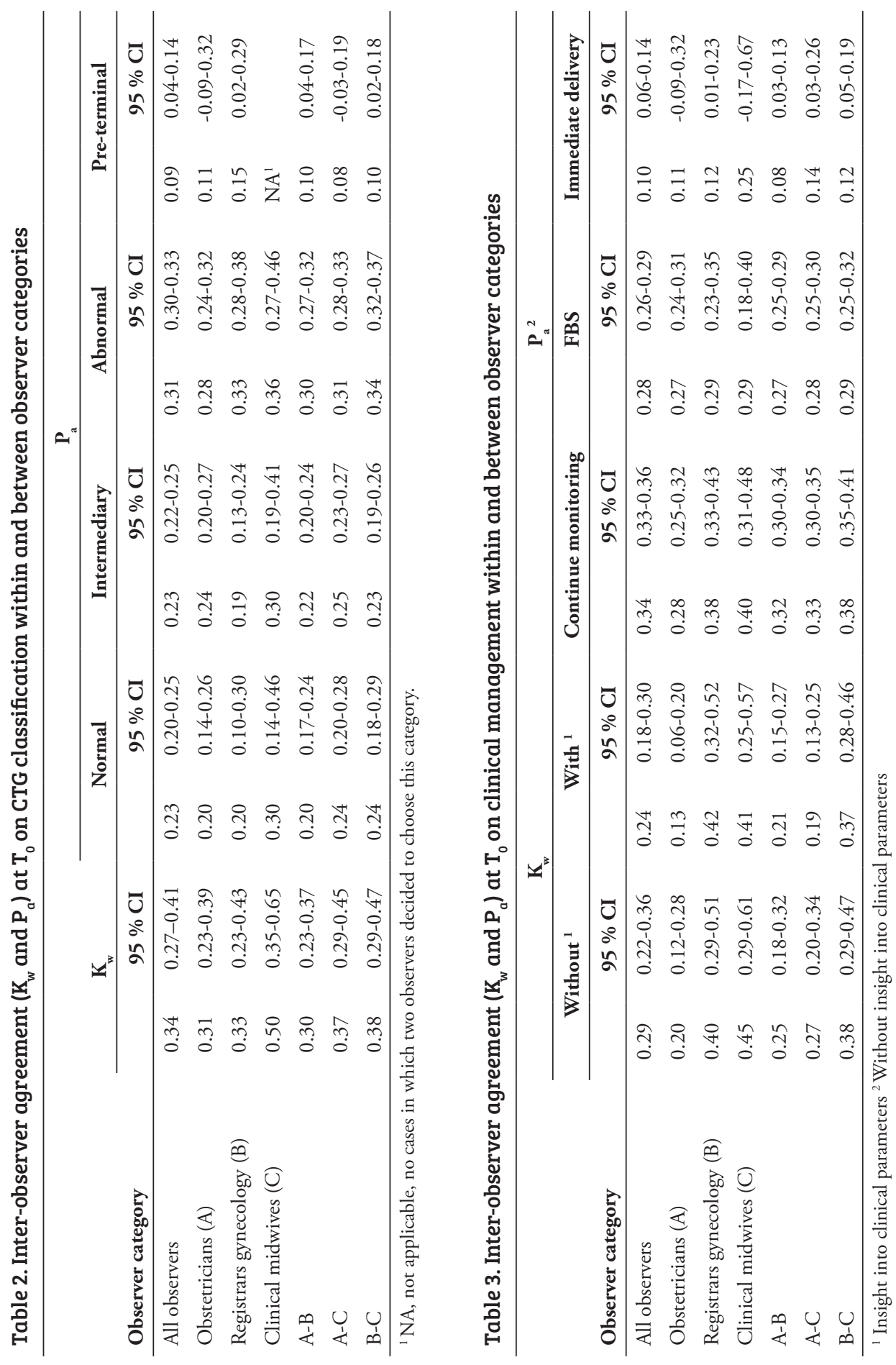
At T1, agreement on CTG classification and clinical management strategy was slightly lower compared to agreement at T0 within observer categories 'clinical midwives' and 'registrars'. Within observer category 'obstetricians' and between observer categories agreement was similar to agreement at T0. Intra-observer agreement on CTG classification and clinical management strategy was fair to good for most observers (Table 4). Intra-observer agreement was best on abnormal CTG patterns and on the clinical management option 'continue monitoring'.

At T0, after the observers had received insight into clinical parameters, inter-observer agreement was slightly lower within and between most observer categories (Table 3). At T1, a similar decrease in inter-observer agreement was seen. The degree to which insight into clinical parameters changed intra-observer agreement differed between the individual observers (Table 5).

At T0, there was consensus in 43 CTG patterns: 4 normal, 12 intermediary and 25 abnormal. In these CTG patterns, the proportion of non-reassuring scalp $\mathrm{pH}$ was 0 $\%, 10 \%$ and $35 \%$, respectively. At T1, there was consensus in 33 CTG patterns: 4 normal, 11 intermediary and 18 abnormal. In these CTG patterns the proportion of non-reassuring scalp pH was $0 \%, 40 \%$ and $23 \%$, respectively. 


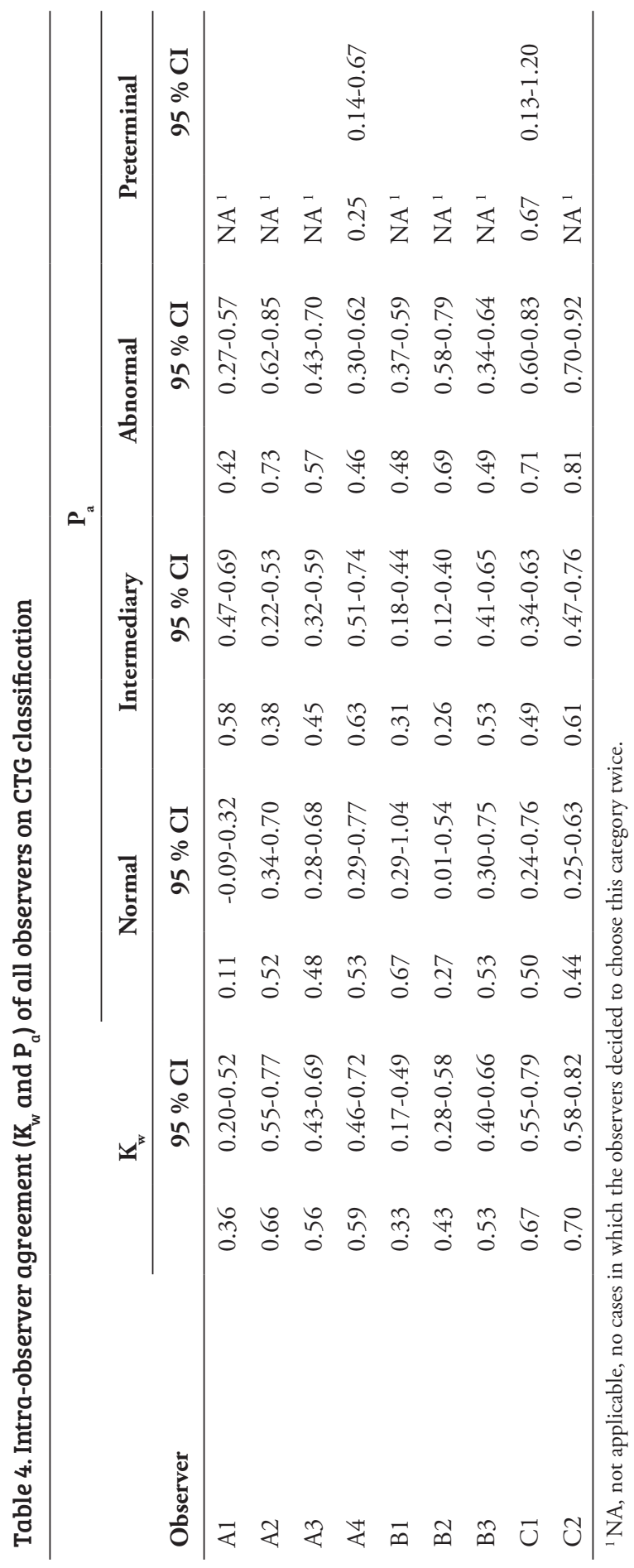




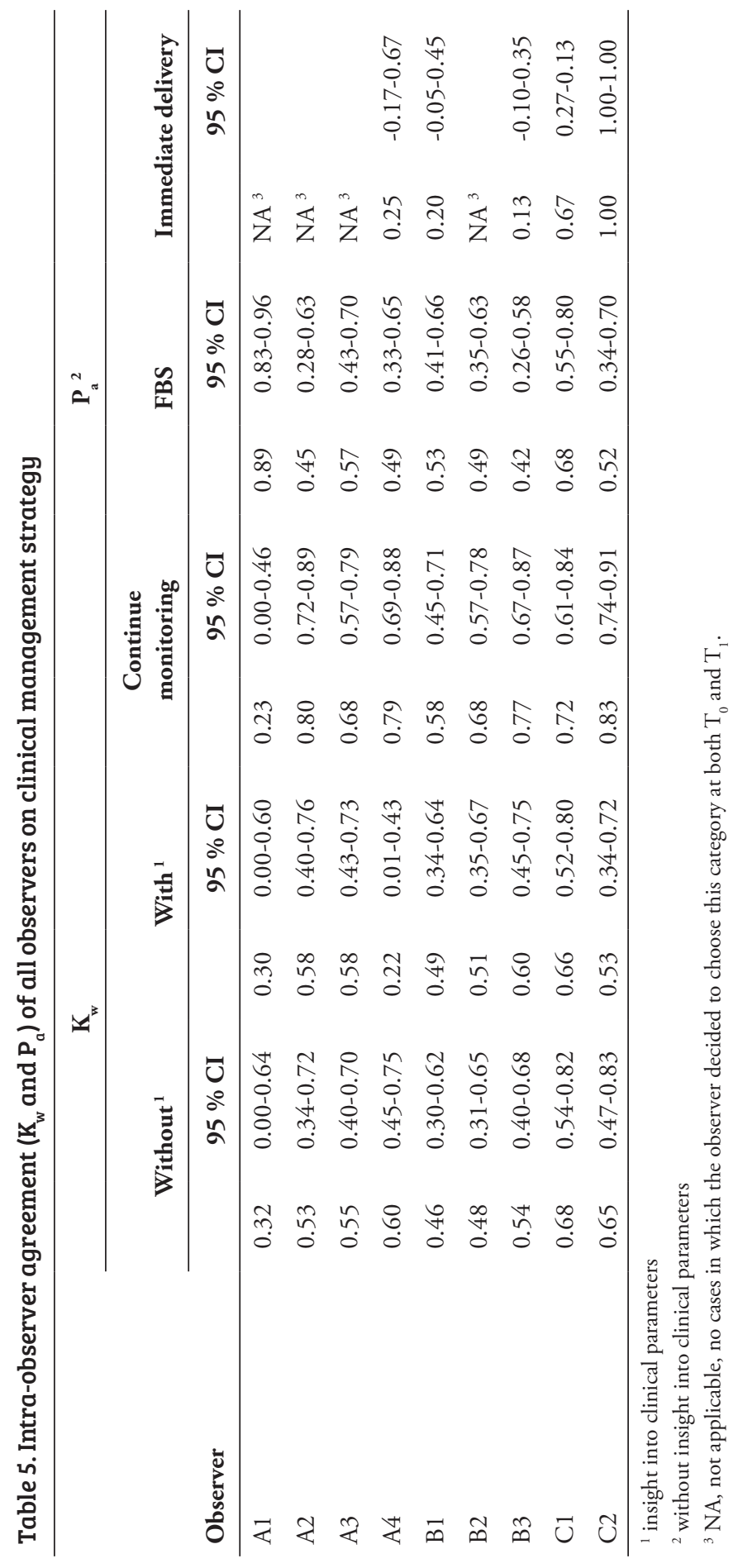




\section{Discussion}

We found considerable variation in the classification of CTG patterns. Observers agreed poorly with each other and fair to good with themselves on CTG classification and clinical management. They achieved highest agreement on abnormal CTG patterns and on the clinical management option 'continue monitoring'.

CTG classification and subsequent clinical management suffer from individual interpretation. Literature is not consistent on the degree of agreement in high risk deliveries, as inter-observer agreement varies from "poor" to "fair to good" (22-24). This inconsistency may be partly dependent upon the used classification system. In correspondence to literature (24), we detected poor inter-observer agreement and "fair to good" intra-observer agreement when classifying CTG patterns according to the FIGO/STAN guidelines. This implies that, even with strict guidelines, the agreement on CTG-classification between observers is poor, although individuals appear to score CTG patterns more consistent.

The level of experience and type of profession may influence CTG interpretation. Westerhuis et al. showed differences indicative for higher rates of agreement in more experienced and recently educated professionals (24). We could not confirm this observation, as our study showed that both clinical midwives and registrars agreed better than the obstetricians. One may speculate on possible explanations: (i) registrars are all trained in the same teaching hospital, while the obstetricians have different backgrounds, (ii) clinical midwives and registrars are more "bed-side" than obstetricians and have more current experience than obstetricians, (iii) the observer category size differed amongst the groups (four obstetricians, three registrars and two clinical midwives) which increases the probability that midwives' fair to good agreement was due to chance.

CTG classification is used to identify those fetuses at risk for metabolic acidosis. Literature reports that the rate of agreement on clinical management correlates to fetal umbilical cord $\mathrm{pH}$ at birth (23). In line with literature, we found that CTG category correlates with scalp pH if several observers agree about the CTG category. However, these results must be interpreted with caution as they are based on a small sample. Therefore, it is still unknown whether better agreements are correlated with 'true' interpretations and if better agreement will improve neonatal outcome. 
Some physicians share the opinion that a CTG can only be assessed adequately with insight into clinical parameters. We did not find literature that investigated the value of additional clinical parameters to CTG interpretation. In our study, the insight into clinical parameters did not influence inter-observer agreement. Possibly, the degree to which observers included the clinical parameters in their CTG assessment was heterogenic. Furthermore, by providing clinical parameters we added more variables about which observers could disagree.

Our study provides additional insight in CTG interpretation. However, some limitations need to be addressed. Firstly, we only included CTG patterns which were formerly clinically classified as indicative for FBS. This may have led to a relatively low number of normal and pre-terminal CTG patterns. As a result, sufficient CTG patterns were available to say something conclusive about the indication to perform FBS with a reasonable total number of CTG patterns (19). Additionally, Westerhuis et al. showed good inter-observer agreement on CTG's classified as normal or (pre) terminal (24), which makes this category of CTG patterns of less interest. Further, all observers were provided with the information it concerned CTG patterns formerly diagnosed as non-reassuring and indicative for FBS. This may have influenced observers' assessments, for example by causing them to choose the clinical management option 'FBS' more often. However, by providing this information we ensured all observers assessed the CTG patterns with the same foreknowledge. Another limitation is the fact only two midwives participated in our study. In an optimal setting, we would have wanted at least three observers in each observer group to achieve a narrow enough confidence interval around $\mathrm{Pa}$ when using 97 CTG patterns. This means the results in the midwives group are more sensitive to observer bias and should therefore be interpreted with care. Finally, observers assessed the CTG patterns using the FIGO/STAN guidelines, while the CTG patterns stem from a period of time in which the FIGO/STAN guidelines were not used at the hospital. Probably, the indication to perform FBS had been more liberal at that time.

Our study showed that interpretation, i.e. classification of CTG patterns remains difficult even when using the FIGO/STAN guidelines. This has clinical implications regarding the use of FBS and fetal monitoring with CTG. Since the positive predictive value of FBS is expected to be low if the a priori chance for a fetus suffering from acidosis is low, we think the indication to perform FBS should be more strictly described to ensure the use of FBS is as efficient as possible. Moreover, physicians should be aware of the fact that there is a relatively high chance their colleagues would classify a CTG pattern differently or would choose another clinical management strategy, underlining the importance of critical discussion between colleagues. There 
is a need for a stricter and better implemented CTG classification system, which may help to increase the rate of agreement on classification and clinical management of CTG patterns. 


\section{References}

(1) Alfirevic Z, Devane D, Gyte GML. Continuous cardiotocography (CTG) as a form of electronic fetal monitoring (EFM) for fetal assessment during labor. Cochrane Database Syst Rev. 2006.

(2) Ayres-de-Campos D, Bernardes J. Twenty-five years after the FIGO guidelines for the use of fetal monitoring: time for a simplified approach? Int J Gynaecol Obstet. 2010 Jul;110(1):1-6.

(3) Ayres-de-Campos D, Bernardes J, Costa-Pereira A, Pereira-Leite L. Inconsistencies in classification by experts of cardiotocograms and subsequent clinical decision. BJOG. 1999;106:1307-10.

(4) Bernardes J, Costa-Pereira A, Ayres-de-Campos D, van Geijn HP, Pereira-Leite L. Evaluation of interobserver agreement of cardiotocograms. Int J Gynaecol Obstet. 1997 Apr;57:33-7.

(5) Figueras F, Albela S, Bonino S, Palacio M, Barrau E, Hernandez S, et al. Visual analysis of antepartum fetal heart rate tracings: inter- and intra-observer agreement and impact of knowledge of neonatal outcome. J Perinat Med. 2005;33:241-5.

(6) Lotgering FK, Wallenburg HC, Schouten HJ. Interobserver and intraobservervariation in the assessment of antepartum cardiotocograms. Am J Obstet Gynecol. 1982;144:701-5.

(7) Devane D, Lalor J. Midwives' visual interpretation of intrapartum cardiotocographs: intra- and inter-observer agreement. J Adv Nurs. 2005;52:133-41.

(8) Mahendru AA, Lees CC. Is intrapartum fetal blood sampling a gold standard diagnostic tool for fetal distress? Eur J Obstet Gynecol Reprod Biol. 2011;156:137-9.

(9) FIGO subcommittee on Standards in Perinatal Medicine. Guidelines for the use of fetal monitoring. Int J Gynaecol Obstet. 1987;25:159-67.

(10) Holzmann M, Cnattingius S, Nordstrom L. Outcome of severe intrapartum acidemia diagnosed with fetal scalp blood sampling. J Perinat Med. 2011 Sep;39(5):545-8.

(11) Westerhuis ME, Strasser SM, Moons KG, Mol BW, Visser GH, Kwee A. [Intrapartum foetal monitoring: from stethoscope to ST analysis of the ECG]. Ned Tijdschr Geneeskd. 2009;153:B259.

(12) Goodwin TM, Milner-Masterson L, Paul RH. Elimination of fetal scalp blood sampling on a large clinical service. Obstet Gynecol. 1994;83:971-4.

(13) Murphy KW, Macdonald D. Fetal blood sampling in Dublin - a year's review. J Obstet Gynaecol. 1990 Jan;10:194-8.

(14) Stein W, Hellmeyer L, Misselwitz B, Schmidt S. Impact of fetal blood sampling on vaginal delivery and neonatal outcome in deliveries complicated by pathologic fetal heart rate: a population based cohort study. J Perinat Med. 2006;34:479-83.

(15) National Collabourating Centre for Women's and Children's Health commissioned by the National Institute for Health and Clinical Excellence. Intrapartum care. London: RCOG Press. 2007.

(16) NVOG. Richtlijn foetale bewaking. 2003. p. 4. 
(17) Amer-Wahlin I, Arulkumaran S, Hagberg H, Marsal K, Visser GH. Fetal electrocardiogram: ST waveform analysis in intrapartum surveillance. BJOG. 2007;114:1191-3.

(18) Heinis AM, Spaanderman ME, Gunnewiek JM, Lotgering FK. Scalp blood lactate for intra-partum assessment of fetal metabolic acidosis. Acta Obstet Gynecol Scand. 2011;90:1107-14.

(19) Grant JM. The fetal heart rate trace is normal, isn't it? Observer agreement of categorical assessments. Lancet. 1991;337:215-8.

(20) Sim J, Wright CC. The kappa statistic in reliability studies: use, interpretation, and sample size requirements. Phys Ther. 2005;85:257-68.

(21) Walter SD, Eliasziw M, Donner A. Sample size and optimal designs for reliability studies. Stat Med. 1998;17:101-10.

(22) Ojala K, Makikallio K, Haapsamo M, Ijas H, Tekay A. Interobserver agreement in the assessment of intrapartum automated fetal electrocardiography in singleton pregnancies. Acta Obstet Gynecol Scand. 2008;87(5):536-40.

(23) Vayssiere C, Tsatsaris V, Pirrello O, Cristini C, Arnaud C, Goffinet F. Inter-observer agreement in clinical decision-making for abnormal cardiotocogram (CTG) during labor: a comparison between CTG and CTG plus STAN. BJOG. 2009 Jul;116(8):1081-8.

(24) Westerhuis ME, van Horen E, Kwee A, van der Tweel I, Visser GH, Moons KG. Interand intra-observer agreement of intrapartum ST analysis of the fetal electrocardiogram in women monitored by STAN. BJOG. 2009;116:545-51.

(25) Verburg BO, Steegers EA, De Ridder M, Snijders RJ, Smith E, Hofman A, et al. New charts for ultrasound dating of pregnancy and assessment of fetal growth: longitudinal data from a population-based cohort study. Ultrasound Obstet Gynecol. 2008 Apr;31(4):388-96.

(26) Visser GH, Eilers PH, Elferink-Stinkens PM, Merkus HM, Wit JM. New Dutch reference curves for birthweight by gestational age. Early Hum Dev. 2009 Dec;85(12):737-44. 


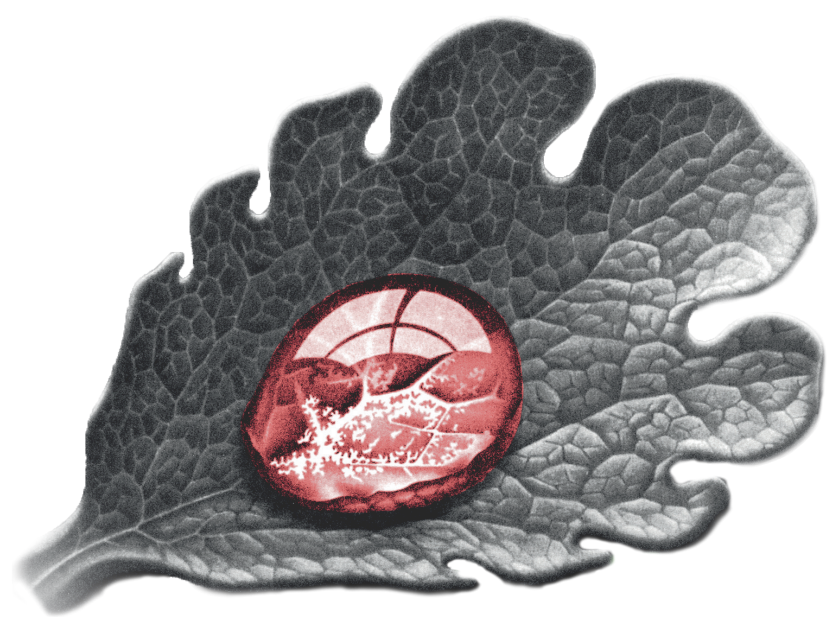




\section{Chapter 3}

SCALP BLOOD LACTATE FOR INTRA-PARTUM ASSESSMENT OF FETAL METABOLIC ACIDOSIS

Ayesha MF Heinis, Marc E Spaanderman, Jacqueline MT Klein Gunnewiek, Fred K Lotgering 


\section{Abstract}

Objective: To study to what extent the fetal scalp blood lactate concentration during labor correlates with fetal scalp $\mathrm{pH}$ and base deficit, and metabolic acidosis at birth, and to suggest lactate cut-off values to serve as indicators for either reassurance or immediate intervention.

Design: A retrospective observational study.

Setting: Labor ward at the Radboud University Medical Centre, Nijmegen, the Netherlands.

Sample: Fetal scalp and cord blood samples with acid-base and lactate values from 486 singleton pregnancies beyond 34 weeks' gestation.

Methods: The relation between lactate, $\mathrm{pH}$ and base deficit (BD) in fetal scalp blood was tested by Spearmans's rho correlation coefficient. Lactate cut-off values indicating either reassuring fetal status or immediate intervention were estimated using percentile distribution and compared to $\mathrm{pH}$ and $\mathrm{BD}$.

Main outcome measures: Metabolic acidosis, defined as umbilical cord artery $\mathrm{pH}$ below 7.05 and base deficit (BD) calculated for the blood compartment above $12 \mathrm{mmol} / \mathrm{l}$.

Results: After 127 (21\%) exclusions, 486 cases were available for analysis. Fetal lactate values increased with evolving metabolic acidosis. Lactate concentration correlated with both $\mathrm{pH}(\mathrm{r}=-0.50, \mathrm{p}<0.01)$ and $\mathrm{BD}(\mathrm{r}=0.48$, $\mathrm{p}<0.01)$. Lactate $<5.4 \mathrm{mmol} / \mathrm{l}$ indicated reassuring fetal status, whereas lactate $\geq 6.6 \mathrm{mmol} / \mathrm{l}$ indicated metabolic acidosis. Fetal lactate correlated better with either the absence or presence of metabolic acidosis at birth than did fetal $\mathrm{pH}$ and $\mathrm{BD}$.

Conclusions: In case of a non-reassuring fetal heart rate, fetal scalp blood lactate provides more accurate information on fetal acid-base status than does $\mathrm{pH}$ and/or BD. 


\section{Introduction}

Intra-partum cardiotocography is a useful screening test for hypoxic/acidotic distress but requires supplementary tests to avoid unnecessary operative delivery (1). Fetal blood sampling (FBS) with $\mathrm{pH}$ analysis is used to identify those fetuses with suspected distress as actually being acidotic (2). When only $\mathrm{pH}$ is used, no differentiation is made between respiratory (CO2 accumulation) and metabolic acidosis (lactate acidosis). However, poor neonatal outcome is associated with metabolic rather than respiratory acidosis $(3,4)$. The base deficit $(\mathrm{BD})$ varies linearly with the degree of metabolic acidosis, whereas it is virtually unaffected by respiratory acidosis (5). Therefore, full blood gas analysis is considered more informative than $\mathrm{pH}$ alone. Lactate may be the better indicator of anaerobic metabolism compared to $\mathrm{BD}$. $\mathrm{BD}$ is calculated from measured $\mathrm{pH}$ and $\mathrm{pCO} 2$ levels and is therefore sensitive to error, whereas lactate is the actual measured end product of anaerobic metabolism.

Analysis of $\mathrm{pH}$ is cumbersome in fetal scalp blood, with or without full blood gas analysis. The amount of blood needed is relatively large $(35-95 \mu \mathrm{L})$, sampling failure rates are high (11-20\%), and the logistics of analysis are time-consuming (20-30 minutes) $(6,7)$. Lactate analysis may be a better alter- native. It requires only a tiny

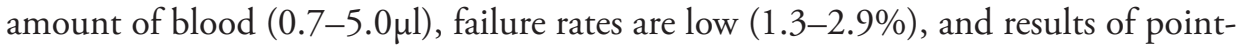
of- care testing devices are quickly available (13-60 seconds) (7-9).

In the assessment of metabolic acidosis after birth, lactate values in arterial cord blood correlate well with $\mathrm{pH}$ and $\mathrm{BD}$ (10). This suggests that the same may be true for fetal scalp lactate concentration as a direct measure of metabolic acidosis during labor and delivery. Although fetal scalp lactate has been compared with fetal scalp $\mathrm{pH}$, little is known about scalp blood lactate in relation to concomitantly analyzed fetal scalp BD $(11,12)$.

This study tested the hypothesis that fetal scalp lactate concentration during labor correlates directly with fetal scalp $\mathrm{pH}$ and $\mathrm{BD}$ and relates to metabolic acidosis at birth. To this end, fetal scalp blood data were analyzed concomitantly for lactate, $\mathrm{pH}$, and $\mathrm{BD}$, and were correlated both with each other and with metabolic acidosis at birth. Fetal scalp blood lactate levels were estimated to serve as indicators for either reassurance or the necessity of immediate intervention. 


\section{Material and methods}

The study was conducted with FBS and cord blood data obtained from women during labor at the Radboud University Nijmegen Medical Center during a four-year period (2004-2007). The Research Ethics Committee at the Radboud University Nijmegen Medical Center approved the study (2011/019).

Deliveries were managed by residents or midwives supervised by a gynecologist. Cardiotocograms were classified and interpreted according to the guidelines of the International Federation of Gynecology and Obstetrics (13). FBS was per- formed on indication by the obstetric caregiver in the case of an intermediary or abnormal cardiotocography trace. Excluded were women with a pregnancy $<34$ weeks, multiple gestation, or non-vertex presentation, incomplete obstetric record, incomplete set of laboratory data on lactate, $\mathrm{pH}$ and $\mathrm{BD}$ in FBS or cord blood. If FBS was performed more than once, only the last sample prior to delivery was included in the analysis.

FBS was carried out by a standard technique (2). After incision of the fetal scalp, blood was collected in heparinized plastic capillaries and sent to the laboratory for immediate full blood gas and lactate analysis on a blood gas analyzer (Rapidlab 860, Siemens Health- care Diagnostics, Deerfield, IL, USA). This required samples $\geq 35 \mu$ l. Base deficit was calculated for the blood compartment $[\mathrm{BD}(\mathrm{B})]$ using the algorithm: $\mathrm{BD}(\mathrm{B})=(1-0.014 \times \mathrm{ctHb})[\mathrm{c} \mathrm{HCO} 3--24.8)+(1.43 \times \mathrm{c} \mathrm{tHb}+7.7)(\mathrm{pH} 7.40)]$. Base deficit for the blood compartment was used, as it was reported to show a closer association with neonatal depression than BD calculated for the extracellular fluid compartment (14).

The lactate values were not available for clinical use, but $\mathrm{pH}, \mathrm{pCO}$, and $\mathrm{BD}$ were. As no intra-partum cut-off values were available for these parameters, only $\mathrm{pH}$ was used for clinical management. Guidelines for fetal $\mathrm{pH}$ were: $\mathrm{pH}>7.25$ normal; continue fetal monitoring, 7.21-7.25 pre-acidemia; consider repeat FBS within 30 minutes, $<7.21$ acidemia; con- sider immediate delivery. Fetal buffer capacity was considered not to be reduced when scalp blood BD was $<8.0 \mathrm{mmol} / \mathrm{l}(4,15)$. A BD value $\geq 10 \mathrm{mmol} / 1$, representing the 85 th per- centile of the study population, was chosen as a marker for the need of immediate intervention.

Metabolic acidosis at birth was systematically evaluated by cord blood sampling immediately after delivery from both the umbilical artery and vein, as previously reported (16). The umbilical vein results were used to verify correct sampling. Only 
data from the umbilical artery were used in the analysis, assuming that this was the best reflection of fetal condition. Metabolic acidosis at birth was defined as $\mathrm{pH}<7.05$ and $\mathrm{BD}>12.0 \mathrm{mmol} / \mathrm{l}$ in umbilical artery blood (17).

Statistical analysis was performed with SPSS 16.0 (SPSS Inc., Chicago, IL, USA). Data are presented as medians with 95 (2.5-97.5) percentile ranges. Data were tested non- parametrically by the Mann-Whitney U-test or chi-squared test whenever applicable and corrected for multiple testing (Bonferroni correction). Relation between two variables was tested using Spearman's rho correlation coefficient. A $\mathrm{p}$-value $<0.05$ was considered significant. To estimate fetal lactate cut-off values, the percentile distribution in the study population was used.

Fetal $\mathrm{pH}$ values, divided as normal, pre-acidemia, and acidemia, are used to guide clinical management. In analogy with that, we chose the lactate value $<75$ th percentile as marker for reassurance and the lactate value $>85$ th percentile as marker for the need of immediate intervention. A lactate value between the 75th and 85th percentile was considered to reflect the intermediate zone in which repeat FBS within 30 minutes might be considered. The 'predictive' proper- ties of lactate, $\mathrm{pH}$, and $\mathrm{BD}$ cut-off values were studied using cross-tabulation. Sensitivity, specificity, positive and negative predictive values were calculated. 


\section{Results}

During the study period, 5448 women were delivered. FBS was performed in 613 (11\%) women. After 127 (21\%) exclusions, 486 cases were available for analysis. Median time from sampling to delivery was 54 (33-105) minutes. Lactate, $\mathrm{pH}$, $\mathrm{BD}$, and $\mathrm{pCO} 2$ values were not normally distributed either in fetal scalp blood or in umbilical artery blood after birth. The intra-partum characteristics of the 486 mother-baby pairs, and lactate, $\mathrm{pH}, \mathrm{BD}$, and $\mathrm{pCO} 2$ values in fetal scalp blood as well as in umbilical artery blood after birth are summarized in Table 1. Seventeen $(3 \%)$ infants had metabolic acidosis at birth.

Table 1. Intra partum characteristics, and fetal scalp and cord blood values for lactate, $\mathrm{pH}$, base deficit and $\mathrm{pCO}_{2}$ of 486 mother-baby pairs.

\begin{tabular}{lc}
\hline Characteristic & Number (\%) or Median (range) \\
\hline Maternal age (years) & $32(28-35)$ \\
Nulliparous & $354(73 \%)$ \\
Gestational age (days) & $284(248-297)$ \\
Meconium stained amniotic fluid & $158(33 \%)$ \\
FBS Lactate (mmol/l) & $3.9(1.7-9.0)$ \\
FBS pH & $7.28(7.08-7.41)$ \\
FBS BD (mmol/l) & $5.8(3.0-13.9)$ \\
FBS pCO 2 (kPa) & $6.1(3.7-9.1)$ \\
Operative delivery* & $282(58 \%)$ \\
Five minute Apgar score $<7$ & $15(3 \%)$ \\
Birthweight (grams) & $3442(2255-4399)$ \\
UA Lactate (mmol/l) & $6.2(2.5-11.1)$ \\
UA pH & $7.19(6.99-7.32)$ \\
UA BD (mmol/l) & $8.3(2.5-15.5)$ \\
UA PCO ${ }_{2}(\mathrm{kPa})$ & $7.5(4.9-10.8)$ \\
Metabolic acidosis at birth ${ }^{1}$ & $17(3 \%)$ \\
\hline
\end{tabular}

Data are numbers and percentage of total (\%), or medians with $2.5^{\text {th }}$ and $97.5^{\text {th }}$ ranges. "Forceps/ventouse or cesarean section. FBS: Fetal blood sample. UA: Umbilical artery. ${ }^{1} \mathrm{pH}<7.05$ and base deficit $>12 \mathrm{mmol} / \mathrm{l}$ in umbilical artery blood. 
Fetal scalp blood lactate correlated significantly with $\mathrm{pH}$ and $\mathrm{BD}(\mathrm{r}=-0.50, \mathrm{p}<0.001$ and $0.48, \mathrm{p}<0.001$, respectively) (Figure 1a, b). Fetal scalp blood samples were categorized by $\mathrm{pH}$ and $\mathrm{BD}$ levels into four groups: group $\mathrm{I}-\mathrm{pH}>7.25$ and $\mathrm{BD}<$ 8; group II $-\mathrm{pH}>7.25$ and $\mathrm{BD} \geq 8$; group III $-\mathrm{pH} \leq 7.25$ and $\mathrm{BD}<8$; group $\mathrm{IV}-\mathrm{pH} \leq 7.25$ and $\mathrm{BD} \geq 8$, indicating nor- mal acid-base status, reduced buffer base, hypercapnia in the absence of metabolic acidosis, and metabolic acidosis, respectively. Thus categorized, the lactate levels showed a significant increase $(\mathrm{p}<0.01)$ with progressing metabolic acidosis (Table 2). Median values in groups I and IV were concordant for absence or presence of metabolic acidosis, respectively, based on lactate, $\mathrm{pH}$, and $\mathrm{BD}$. In groups II and III, the median lactate level appeared to be a better indicator of a mild degree of metabolic acidosis compared with BD.

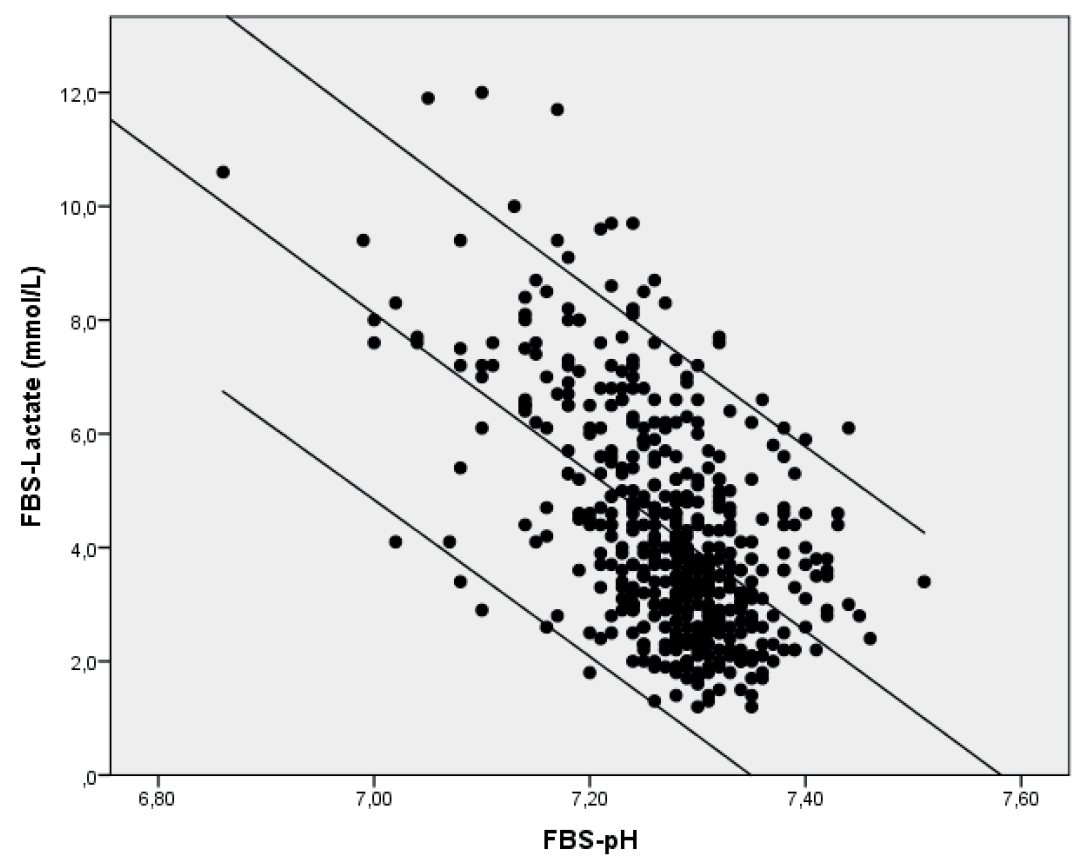

Figure 1a. Relation between lactate and $\mathrm{pH}(\mathrm{r}=-0.50, \mathrm{p}<0.01)$ in fetal scalp blood. 


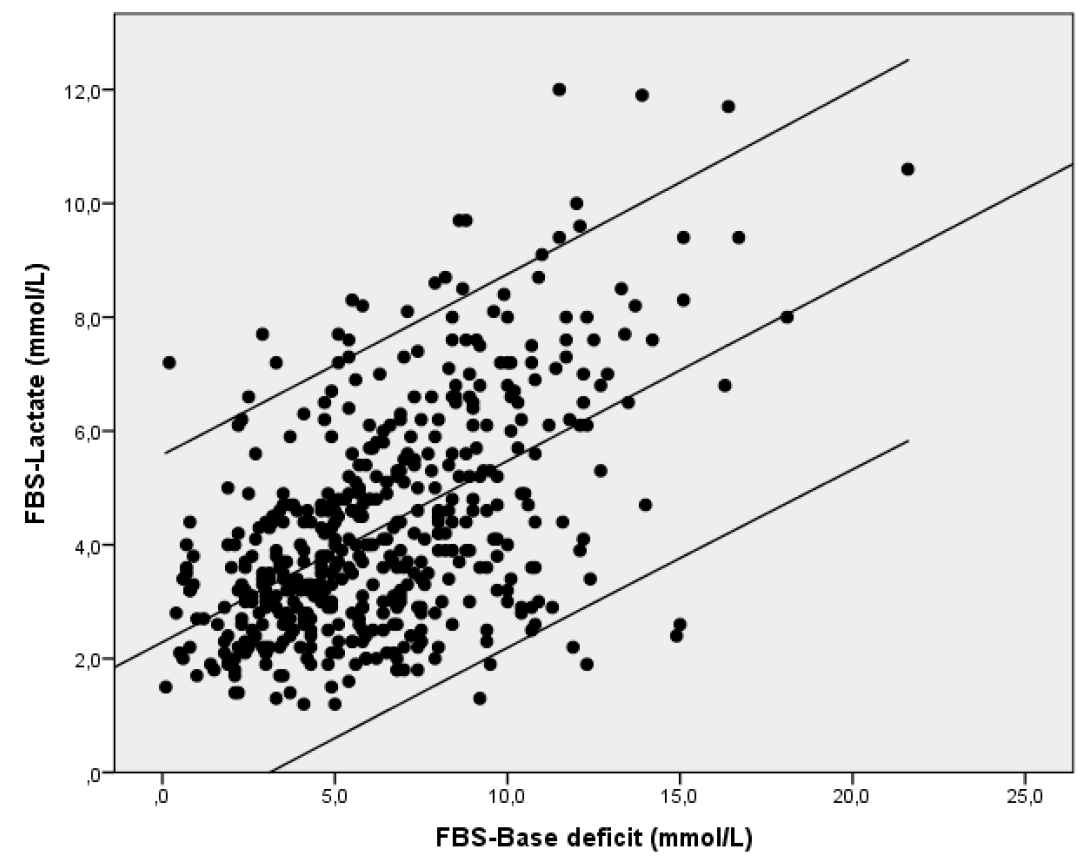

Figure 1b. Relation between lactate and $\mathrm{BD}(\mathrm{r}=0.48, \mathrm{p}<0.01)$ in fetal scalp blood.

Umbilical artery blood after birth was more acidic than fetal scalp blood, as shown by the median values of lactate (6.2 vs. $3.9 \mathrm{mmol} / \mathrm{l}), \mathrm{pH}(7.19$ vs. 7.28$)$, and BD ( 8.3 vs. $5.8 \mathrm{mmol} / \mathrm{l}$ ), and pCO2 was higher (7.5 vs. $6.1 \mathrm{kPa}$ ) (Table 1). Umbilical artery lactate was significantly related to $\mathrm{pH}(-0.50, \mathrm{p}<0.01)$ and $\mathrm{BD}(0.55, \mathrm{p}<0.01)$ (Figure 2a, b). Categorized by BD and pH levels, the lactate level in umbilical artery blood showed a significant increase $(\mathrm{p}<0.01)$ with progressing metabolic acidosis (Table 3), except in group III, which contained only three samples, with the highest lactate values, and in group IV, which had more severe acidosis. Median values in groups I and IV were concordant for absence or presence of metabolic acidosis based on lactate, $\mathrm{pH}$, and $\mathrm{BD}$. Group II showed relatively high BD, suggestive of metabolic acidosis, as a result of relatively low pCO2. Group III showed relatively low BD, suggestive of absence of metabolic acidosis as a result of relatively high pCO2. In groups II and III, the median lactate level appeared to be a better indicator of degree of metabolic acidosis compared with BD. 
Table 2. Fetal scalp blood lactate, $\mathrm{pH}$, base deficit and $\mathrm{pCO}_{2^{\prime}}$ categorized according to $\mathrm{pH}$ $(7,25)$ and base deficit $(8 \mathrm{mmol} / \mathrm{l})$ thresholds of reassuring fetal acid/base status.

\begin{tabular}{|c|c|c|c|c|}
\hline \multirow[b]{3}{*}{$\mathbf{N}$} & \multicolumn{4}{|c|}{ Fetal scalp blood } \\
\hline & \multicolumn{2}{|c|}{$\mathrm{pH}>7.25$} & \multicolumn{2}{|c|}{$\mathrm{pH} \leq 7.25$} \\
\hline & $\begin{array}{c}\mathrm{I} \\
\mathrm{BD}<8 \\
272\end{array}$ & $\begin{array}{c}\text { II } \\
\text { BD } \geq 8 \\
46\end{array}$ & $\begin{array}{c}\text { III } \\
\mathrm{BD}<8 \\
69\end{array}$ & $\begin{array}{c}\text { IV } \\
\mathrm{BD} \geq 8 \\
99\end{array}$ \\
\hline Lactate $(\mathrm{mmol} / \mathrm{l})$ & $3.3(1.5-6.7)$ & $3.9(1.4-8.5)^{*}$ & $4.5(2.0-8.3)^{*}$ & $6.6(2.5-11.8)^{*}$ \\
\hline $\mathrm{pH}$ & $7.31(7.26-7.43)$ & $7.29(7.26-7.38)^{*}$ & $7.24(7.13-7.25)^{*}$ & $7.18(7.00-7.25)^{*}$ \\
\hline $\mathrm{BD}(\mathrm{mmol} / \mathrm{l})$ & $4.3(0.6-7.5)$ & $9.2(8.0-14.5)^{*}$ & $6.0(3.0-7.9)^{*}$ & $10.4(8.0-17.4)^{*}$ \\
\hline $\mathrm{pCO}_{2}(\mathrm{kPa})$ & $5.9(3.9-7.4)$ & $4.5(1.9-5.7)^{*}$ & $7.3(6.0-9.9)^{*}$ & $6.5(4.0-10.3)^{*}$ \\
\hline
\end{tabular}

Data are presented as median values with $2.5^{\text {th }}$ and $97.5^{\text {th }}$ centile ranges; ${ }^{*}$ indicates $\mathrm{p}<0.01$ compared to group I $(\mathrm{pH}>7.25$ and base deficit $<8 \mathrm{mmol} / \mathrm{l})$.

Table 3. Umbilical artery blood lactate, $\mathrm{pH}$, base deficit and $\mathrm{pCO}_{2}$, categorized according to $\mathrm{pH}(7.05)$ and base deficit (12 mmol/l) thresholds for metabolic acidosis at birth.

\begin{tabular}{lcccc}
\hline & \multicolumn{4}{c}{ Umbilical artery blood } \\
\cline { 2 - 5 } & $\mathbf{I}$ & $\mathbf{p H} \geq \mathbf{7 . 0 5}$ & $\mathbf{p H}<\mathbf{7 . 0 5}$ \\
\cline { 2 - 5 } & $\mathbf{B D} \leq \mathbf{1 2}$ & $\mathbf{I I}$ & $\mathbf{I I I}$ & $\mathbf{I V}$ \\
$\mathbf{N}$ & $\mathbf{4 4 3}$ & $\mathbf{B D}>\mathbf{1 2}$ & $\mathbf{B D} \leq \mathbf{1 2}$ & $\mathbf{B D}>\mathbf{1 2}$ \\
\hline Lactate $(\mathrm{mmol} / \mathrm{l})$ & $6.0(2.4-10.4)$ & $8.7(2.6-14.5)^{*}$ & $10.7(6.9-10.9)$ & $9.1(5.8-17.8)^{*}$ \\
$\mathrm{pH}$ & $7.20(7.08-7.32)$ & $7.09(7.05-7.20)^{*}$ & $7.04(6.92-7.04)^{*}$ & $6.97(6.71-7.04)^{*}$ \\
$\mathrm{BD}(\mathrm{mmol} / \mathrm{l})$ & $8.0(2.5-11.5)$ & $13.4(12.1-15.5)^{*}$ & $11.1(10.9-11.3)^{*}$ & $16.4(13.5-23.2)^{*}$ \\
$\mathrm{PCO}(\mathrm{kPa})$ & $7.5(4.9-9.9)$ & $7.5(4.8-9.5)$ & $10.8(10.8-16.1)^{*}$ & $9.8(6.3-16.9)^{*}$ \\
\hline $\begin{array}{l}\text { Data are presented as median values and } 2.5^{\text {th }} \text { and } 97.5^{\text {th }} \text { centile ranges; }{ }^{*} \text { indicates } \mathrm{p}<0.01 \text { compared to group I } \\
(\mathrm{pH} \geq 7.05 \text { and BD } \leq 12 \text { mmol/l). }\end{array}$ & & & $\mathbf{3}$
\end{tabular}

The 25th percentile for FBS $\mathrm{pH}$ was 7.24 and the 75 th percentile for $\mathrm{BD}$ was 8.4 $\mathrm{mmol} / \mathrm{l}$. These values are close to commonly accepted thresholds for reassuring fetal status $(\mathrm{pH}>7.25, \mathrm{BD}<8.0 \mathrm{mmol} / \mathrm{l})$, for which expectant management is considered safe $(2,4,15)$. The 75 th percentile for lactate was $5.4 \mathrm{mmol} / \mathrm{l}$ and the 75 th percentile for $\mathrm{pCO} 2$ was $6.8 \mathrm{kPa}$. In analogy with the safety margins for $\mathrm{pH}$ and $\mathrm{BD}$, for lactate we chose the 75 th percentile value as the threshold for reassuring fetal status. 


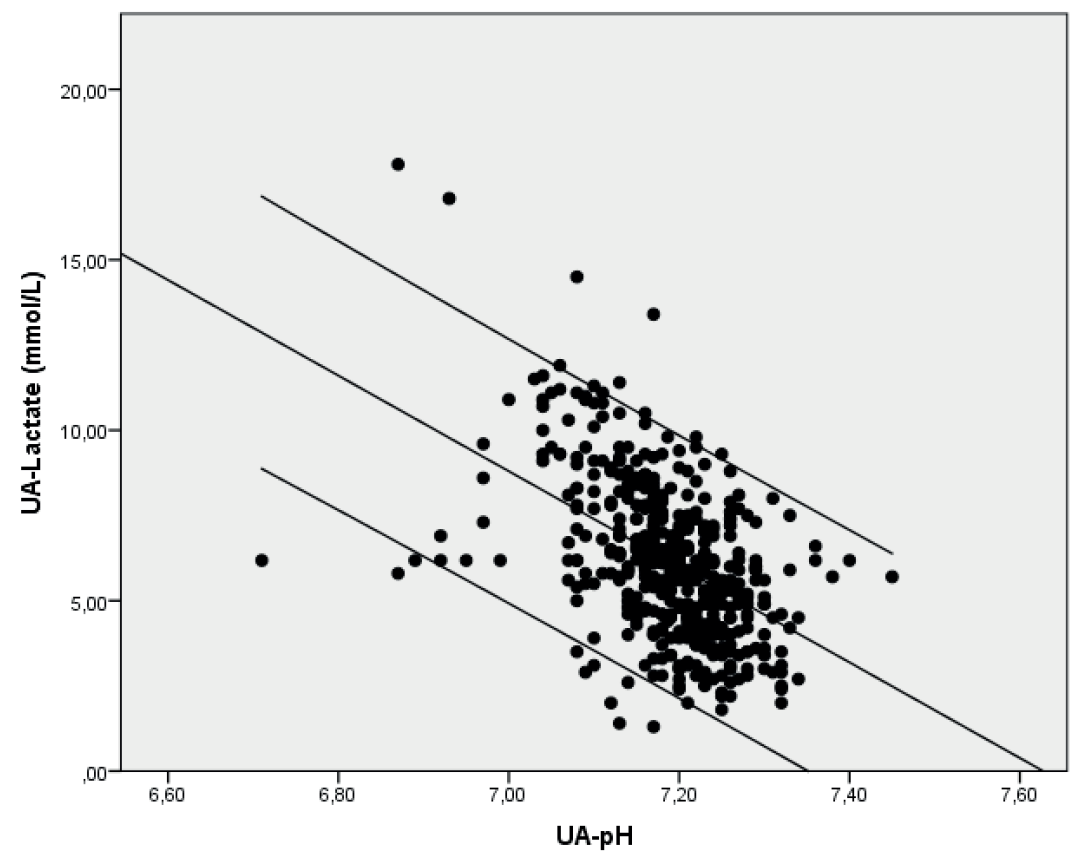

Figure 2a. Relation between lactate and $\mathrm{pH}(\mathrm{r}=-0.50, \mathrm{p}<0.01)$ in umbilical artery blood.

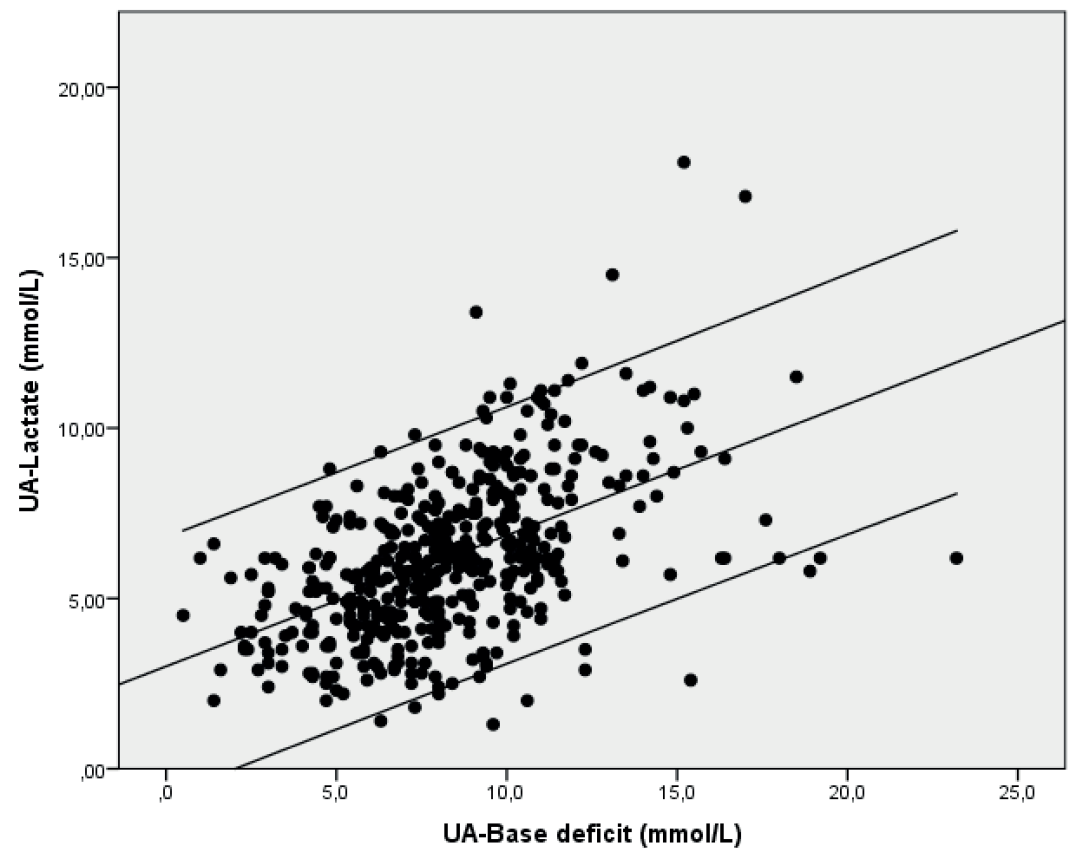

Figure 2b. Relation between lactate and $\mathrm{BD}(\mathrm{r}=0.55, \mathrm{p}<0.01)$ in umbilical artery blood. 
We compared this threshold $(5.4 \mathrm{mmol} / \mathrm{l})$ with existing thresholds for fetal scalp blood of $\mathrm{pH}$ (7.25), $\mathrm{BD}(8.0 \mathrm{mmol} / \mathrm{l})$, and $\mathrm{pH}$ and $\mathrm{BD}$ combined, and related those to the absence of metabolic acidosis at birth (Table 4). Lactate $<5.4 \mathrm{mmol} / \mathrm{l}$ had a sensitivity of $77 \%$, a specificity of $88 \%$, a positive predictive value of $99 \%$, and a negative predictive value of $12 \%$ for reassuring fetal status. Compared to $\mathrm{pH}$, $\mathrm{BD}$, and $(\mathrm{pH}+\mathrm{BD})$, lactate was a better marker of reassuring fetal status. The 15 th percentile for $\mathrm{pH}$ was 7.20 . This value is identical to the commonly accepted threshold for immediate intervention (2). The 85 th percentile for lactate was $6.6 \mathrm{mmol} / \mathrm{l}$, for $\mathrm{BD} 10.0 \mathrm{mmol} / \mathrm{l}$, and for $\mathrm{pCO} 27.3 \mathrm{kPa}$. In analogy with the intervention margin for $\mathrm{pH}$, we chose the 85 th percentile values for lactate and $\mathrm{BD}$ as thresholds for immediate intervention.

Table 4. Test characteristics of threshold values for fetal scalp pH (> 7.25), base deficit $<$ $8 \mathrm{mmol} / \mathrm{l})$ and lactate $(<5.4 \mathrm{mmol} / \mathrm{l})$ in association with absence of acidosis $(\mathrm{pH} \geq 7.05)$ at birth.

\begin{tabular}{lcccc}
\hline $\begin{array}{l}\text { Absent acidosis } \\
\text { at birth }^{*}\end{array}$ & Lactate $<5, \mathbf{4}$ & $\mathbf{p H}>\mathbf{7 . 2 5}$ & $\mathbf{B D}<\mathbf{8}$ & $\mathbf{p H}>\mathbf{7 . 2 5}$ and $\mathbf{B D}<\mathbf{8}$ \\
\hline Sensitivity (95\%CI) & $77(73-81)$ & $67(62-72)$ & $72(65-79)$ & $58(52-64)$ \\
Specificity (95\%CI) & $88(85-91)$ & $88(84-92)$ & $76(69-82)$ & $88(84-92)$ \\
PPV (95\%CI) & $99(98-100)$ & $99(98-100)$ & $99(97-100)$ & $99(98-100)$ \\
NPV (95\%CI) & $12(9-15)$ & $9(6-12)$ & $9(5-13)$ & $7(4-10)$ \\
\hline
\end{tabular}

${ }^{*} \mathrm{pH}<7.05$ and base deficit $>12 \mathrm{mmol} / \mathrm{l}$ in umbilical artery blood. PPV: Positive predictive value, NPV: Negative predictive value.

We compared the new fetal scalp thresholds for immediate intervention (lactate 6.6 $\mathrm{mmol} / \mathrm{l}, \mathrm{BD} 10.0 \mathrm{mmol} / \mathrm{l}$ ) with the existing threshold for fetal scalp blood $\mathrm{pH}(7.20)$, and related those to the presence of metabolic acidosis at birth (Table 5). Lactate $\geq 6.6 \mathrm{mmol} / \mathrm{l}$ had a sensitivity of $71 \%$, a specificity of $87 \%$, a positive predictive value of $16 \%$, and a negative predictive value of $99 \%$. Compared with $\mathrm{pH}, \mathrm{BD}$, and $(\mathrm{pH}+\mathrm{BD})$, lactate was a better marker for the need of immediate intervention.

There were 17 individual cases of metabolic acidosis at birth (umbilical artery $\mathrm{pH}$ $<7.05$ and $\mathrm{BD}>12 \mathrm{mmol} / \mathrm{l}$ ). These are shown in Table $\mathbf{6}$ in order of sampling-todelivery interval. There were no acute obstetric complications. Of the 17 cases, 12 cases were 'correctly identified' by lactate by the marker for immediate intervention ( $\geq 6.6 \mathrm{mmol} / \mathrm{l})$, seven by $\mathrm{pH}(\leq 7.20)$, and nine by $\mathrm{BD}(\geq 10 \mathrm{mmol} / \mathrm{l})$. Five cases were 'correctly identified' by all immediate intervention markers. Two cases were 
'incorrectly identified' by lactate by the reassurance marker $(<5.4 \mathrm{mmol} / \mathrm{l})$, two by $\mathrm{pH}(>7.25)$, and four by BD $(<8.0 \mathrm{mmol} / \mathrm{l})$. One case was 'incorrectly identified' by all reassurance markers. This suggested that lactate was as good a marker as $\mathrm{pH}$ and $\mathrm{BD}$ for the need of immediate intervention.

Table 5. Test characteristics of threshold values for fetal scalp pH ( $\leq 7.20)$, base deficit ( $\geq 10 \mathrm{mmol} / \mathrm{l}$ ) and lactate ( $\geq 6.6 \mathrm{mmol} / \mathrm{l}$ ) in association with metabolic acidosis $(\mathrm{pH}<7.05$ and base deficit $>12 \mathrm{mmol} / \mathrm{l}$ ) at birth.

\begin{tabular}{lcccc}
\hline $\begin{array}{l}\text { Metabolic acidosis } \\
\text { at birth* }\end{array}$ & Lactate $\geq \mathbf{6 . 6}$ & $\mathbf{p H} \leq \mathbf{7 . 2 0}$ & $\mathbf{B D} \geq \mathbf{1 0}$ & $\mathbf{p H} \leq \mathbf{7 . 2 0}$ and $\mathbf{B D} \geq \mathbf{1 0}$ \\
\hline Sensitivity $(95 \% \mathrm{CI})$ & $71(61-81)$ & $41(30-52)$ & $53(42-64)$ & $59(50-68)$ \\
Specificity $(95 \% \mathrm{CI})$ & $87(79-95)$ & $86(77-94$ & $86(78-94)$ & $80(72-88)$ \\
$\mathrm{PPV}(95 \% \mathrm{CI})$ & $16(8-24)$ & $9(2-16)$ & $12(5-19)$ & $9(6-12)$ \\
$\mathrm{NPV}^{2}(95 \% \mathrm{CI})$ & $99(97-100)$ & $98(95-100)$ & $98(95-100)$ & $98(95-100)$ \\
\hline
\end{tabular}

${ }^{*} \mathrm{pH}<7.05$ and base deficit $>12 \mathrm{mmol} / \mathrm{l}$ in umbilical artery blood. PPV: Positive predictive value; NPV: Negative predictive value.

In the nine cases with metabolic acidosis at birth with a sampling-to-delivery interval $\leq 60$ minutes, there was no difference between lactate, $\mathrm{pH}$, and $\mathrm{BD}$ in the number of cases 'correctly identified' by the marker for immediate intervention (six cases by lactate, $\mathrm{pH}$, and $\mathrm{BD}$ ) or 'incorrectly identified' by the reassurance marker (one case by lactate, none by $\mathrm{pH}$, and one by $\mathrm{BD}$ ). From the eight cases with a sampling-todelivery interval > 60 minutes, six (by lactate), one (by $\mathrm{pH}$ ), and three cases (by BD) were 'correctly identified' by the marker for immediate intervention. One case was 'incorrectly identified' by lactate, two by $\mathrm{pH}$, and two by $\mathrm{BD}$ by the reassurance marker.

The majority of infants (12/17) who had metabolic acidosis at birth did not need to be admitted to intensive care and their neonatal course was uncomplicated. Of the five infants who were admitted to the neonatal intensive care, all but one appeared neurologically normal at discharge. This infant suffered from meconium aspiration and was severely anemic ( $\mathrm{Hb} 4.9 \mathrm{mmol} / \mathrm{l})$ at birth, apparently as a result of fetomaternal hemorrhage. In this case, the FBS-lactate, $\mathrm{pH}$, and $\mathrm{BD}$ values all correctly indicated the need for immediate intervention. 
Table 6. Seventeen cases of metabolic acidosis at birth (umbilical artery $\mathrm{pH}<7.05$ and base deficit $>12 \mathrm{mmol} / \mathrm{l})$, tabulated in order of time interval between fetal blood sampling and delivery.

\begin{tabular}{|c|c|c|c|c|c|c|c|c|c|c|c|c|c|}
\hline $\begin{array}{l}\dot{\delta} \\
\dot{z} \\
\tilde{y} \\
\tilde{J}\end{array}$ & 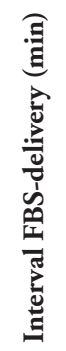 & 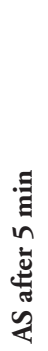 & 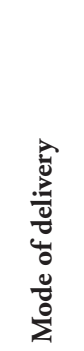 & 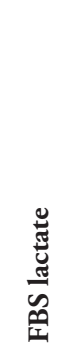 & 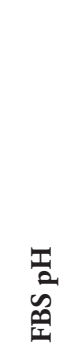 & 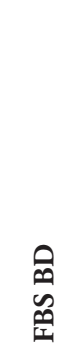 & 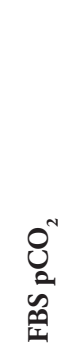 & 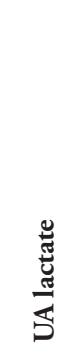 & $\frac{\pi}{2}$ & 用 & 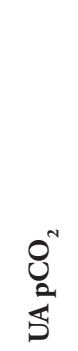 & 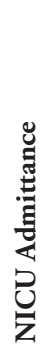 & 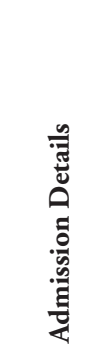 \\
\hline 1 & 18 & 8 & AVD & 8.3 & 7.02 & 15.1 & 9.0 & 9.3 & 7.04 & 15.7 & 7.7 & - & \\
\hline 2 & 20 & 6 & AVD & 11.9 & 7.05 & 13.9 & 8.6 & 11.5 & 7.03 & 18.5 & 6.3 & + & MAS \\
\hline 3 & 21 & 9 & AVD & 6.5 & 7.20 & 9.0 & 6.6 & 10.0 & 7.04 & 15.3 & 7.9 & - & - \\
\hline 4 & 23 & 9 & AVD & 3.4 & 7.08 & 12.4 & 8.6 & 6.2 & 6.99 & 16.4 & 9.1 & - & - \\
\hline 5 & 32 & 6 & AVD & 5.9 & 7.25 & 7.2 & 6.2 & 8.6 & 6.97 & 14.0 & 11.8 & + & - \\
\hline 6 & 35 & 7 & AVD & 6.6 & 7.23 & 10.1 & 5.5 & 7.3 & 6.97 & 17.6 & 8.8 & - & ARPKD \\
\hline 7 & 37 & 7 & CS & 9.4 & 6.99 & 15.1 & 10.0 & 6.2 & 6.89 & 18.0 & 12.0 & + & MAS \\
\hline 8 & 48 & 3 & SVD & 6.8 & 7.25 & 8.5 & 5.7 & 5.8 & 6.87 & 18.9 & 12.3 & - & - \\
\hline 9 & 60 & 7 & CS & 8.0 & 7.14 & 11.7 & 7.0 & 10.9 & 7.00 & 14.8 & 9.8 & - & GBS \\
\hline 10 & 63 & 5 & CS & 6.9 & 7.18 & 10.8 & 6.3 & 11.6 & 7.04 & 13.5 & 9.2 & + & MAS \\
\hline 11 & 70 & 7 & CS & 6.5 & 7.22 & 12.2 & 4.8 & 9.1 & 7.04 & 16.4 & 7.5 & - & GBS \\
\hline 12 & 72 & 8 & CS & 9.6 & 7.21 & 12.1 & 5.0 & 17.8 & 6.87 & 15.2 & 15.5 & - & - \\
\hline 13 & 75 & 9 & CS & 8.2 & 7.24 & 5.8 & 7.2 & 6.5 & 6.71 & 23.2 & 16.9 & + & GBS \\
\hline 14 & 82 & 8 & CS & 9.7 & 7.24 & 8.8 & 5.8 & 6.2 & 6.92 & 19.2 & 9.5 & - & - \\
\hline 15 & 91 & 9 & CS & 9.7 & 7.22 & 8.6 & 6.4 & 16.8 & 6.93 & 17.0 & 11.0 & - & - \\
\hline 16 & 94 & 9 & SVD & 4.0 & 7.28 & 5.6 & 6.1 & 9.6 & 6.97 & 14.2 & 11.6 & - & - \\
\hline 17 & 130 & 8 & AVD & 6.6 & 7.26 & 7.3 & 5.9 & 6.3 & 6.95 & 16.3 & 10.9 & - & - \\
\hline
\end{tabular}

Values correctly indicating immediate intervention at the time of sampling are green; values falsely indicating reassurance are red. AS, Apgarscore; AVD, assisted vaginal delivery; CS, cesarean section; SVD, spontaneous vaginal delivery; UA, umbilical artery; UV, Umbilical vein; NICU, neonatal intensive care unit; MAS, meconium aspiration syndrome; ARPKD, autosomal recessive polycystic kidney disease; GBS, group B streptococcus infection. 


\section{Discussion}

When Saling first introduced the technique of FBS, only $\mathrm{pH}$ was measured (2). $\mathrm{pH}$ alone provides little information about the duration and extent of hypoxia/metabolic acidemia. Transient respiratory acidemia with high $\mathrm{pCO} 2$ is a common feature of normal labor and correlates poorly with neonatal out- come $(3,18)$. In contrast, severe metabolic acidosis represents tissue hypoxia and correlates well with neonatal multi-organ dysfunction and impaired neurodevelopment $(4,19)$.

We used severe metabolic acidosis in umbilical artery blood as a proxy for poor clinical perinatal outcome. One objection to that might be that even marked acidosis may not necessarily be harmful as long as oxygenation is maintained. Although true under experimental conditions in which oxygenation can be artificially adjusted, in everyday obstetrics practice the common reason for metabolic acidosis is underperfusion on the maternal or fetal side, which is generally associated with poor oxygenation. Therefore, we feel that severe metabolic acidosis in umbilical cord blood is the best objective marker of poor neonatal outcome available.

Base deficit is used to quantify metabolic acidosis, as it accounts for both the carbon and the non-carbon buffering systems (5). Full blood gas analysis requires a relatively large amount of blood, which is difficult to obtain by FBS, therefore resulting in rather high failure rates (7). Apart from this practical drawback, for various reasons $\mathrm{BD}$ values may not be the best representative of fetal metabolic status. $\mathrm{BD}$ is not measured but is calculated using $\mathrm{pH}$ and $\mathrm{pCO} 2$ values. As $\mathrm{CO} 2$ can cross the placenta rapidly when perfusion is restored, $\mathrm{pCO} 2$ and therefore $\mathrm{BD}$ can fluctuate. In addition, air contamination in FBS may produce incorrect pCO2 levels and consequently false $\mathrm{BD}$ values. Moreover, the algorithms used for $\mathrm{BD}$ calculation are attuned to adult hemoglobin type, oxygen saturation, and temperature, and not to fetal blood composition, thus causing a systematic error in BD calculation (20). Although one can attempt to correct or exclude any unlikely values, in the absence of generally agreed rules to do so, we chose to report on all values as clinically used.

Lactate is the major end product of anaerobic metabolism and can be measured in a tiny amount of blood, which is practical in FBS. It has been well established by animal and human studies that fetal scalp blood lactate levels adequately represent lactate levels in the fetal central circulation and that fetal lactate production is endogenous in origin $(21,22)$. In recent years, lactate has been shown to be a good predictor of severe morbidity in neonates and in intra-partum surveil- lance $(12,17,23,24)$. 
Our study shows that lactate gradually increases with progressing metabolic acidosis and was a better marker than $\mathrm{pH}$ and/or $\mathrm{BD}$, both of reassurance and of the need for immediate intervention.

The literature provides generally accepted cut-off values for $\mathrm{pH}$, with $\mathrm{pH}>7.25$ indicating reassurance and $\mathrm{pH} \leq 7.20 \mathrm{immediate}$ intervention. This coincided in our study with reassurance at the 25 th percentile and immediate intervention at the 15 th percentile, indicating that $75 \%$ of cases were reassuring, $10 \%$ were inconclusive, and $15 \%$ needed immediate intervention on the basis of $\mathrm{pH}$ alone. Although the validity of the traditional $\mathrm{pH}$ cut-off values may be questioned, given the distribution we chose to align our lactate and BD cut-off values on a percentile distribution equal to that of the generally accepted $\mathrm{pH}$ values.

For the purpose of the study, a fetal scalp blood BD value $<8.0 \mathrm{mmol} / \mathrm{l}$ was considered reassuring. This threshold is based on studies in neonatal cord artery blood, as the literature does not report generally accepted BD cut-off values in fetal scalp blood. However, damaging metabolic acidosis is likely to occur only at BD values at birth of $>12-16 \mathrm{mmol} / \mathrm{l}(4,25)$. Umbilical cord blood is known to be generally more acidic than fetal scalp blood, as the fetus becomes gradually more acidic during the course of labor and delivery (26). If one aims for prevention rather than prediction of metabolic acidosis at birth, BD in fetal scalp should be well below $12.0 \mathrm{mmol} / \mathrm{l}$ to provide reassurance. In our study, a fetal scalp $\mathrm{BD}<8.0 \mathrm{mmol} / \mathrm{L}$ represented $75 \%$ of reassuring cases, a BD between 8.0 and $10.0 \mathrm{mmol} / \mathrm{l}$ the $10 \%$ inconclusive cases, and a $\mathrm{BD} \geq 10.0 \mathrm{mmol} / \mathrm{l}$ the $15 \%$ fetuses in need for immediate intervention.

In our study a lactate value $<5.4 \mathrm{mmol} / \mathrm{l}$ indicated the $75 \%$ of reassuring cases, lactate between 5.4 and $6.6 \mathrm{mmol} / \mathrm{l}$ the $10 \%$ with inconclusive fetal status, and lactate $\geq$ $6.6 \mathrm{mmol} / 1$ the $15 \%$ of fetuses in need of immediate intervention because of marked metabolic acidosis. These lactate cut-off values should not be taken to represent universal absolute threshold values for at least two reasons. Firstly, the cut-off values were estimated on the basis of a retrospective cohort and need confirmation in an independent prospective cohort. Secondly, in the absence of standard calibration techniques between laboratories and devices, our absolute values are valid only with the Rapidlab-860. Nonetheless, fetal lactate concentration not only has the theoretical advantage of measuring the product of metabolic acidosis directly as well as the practical advantage of smaller sample volume and fewer failures, but, as our study shows, it also holds the promise of better test characteristics compared with $\mathrm{pH}, \mathrm{BD}$, and $(\mathrm{pH}+\mathrm{BD})$ combined. 
Intra-partum cardiotocography is a screening test which has high sensitivity to detect fetuses at risk of hypoxemia and subsequent acidosis but rather poor specificity (1). FBS may either provide reassurance or provide additional proof that immediate delivery is needed. In our study, FBS was per- formed in $11 \%$ of all deliveries, reflecting the high level of suspicion in an academic training hospital that a fetal heart rate trace is non-reassuring. The use of FBS in obstetric routine varies widely, from 3 to $15 \%$ in the Netherlands (27) and Sweden (28). To ensure that the threshold values for lactate are valid in both high- and low-risk populations, prospective validation in these populations is needed.

A lactate value $<5.4 \mathrm{mmol} / \mathrm{l}$ indicated reassuring fetal status, whereas a value $\geq 6.6 \mathrm{mmol} / \mathrm{l}$ indicated metabolic acidosis. If $\mathrm{FBS}$ is to provide reassurance, high specificity is important because the intra-partum cardiotocogram already has high sensitivity. As a marker for reassurance, lactate $<5.4, \mathrm{pH}>7.25$, and $(\mathrm{pH}>$ $7.25+\mathrm{BD}<8)$ had equal specificity $(88 \%)$. BD alone performed less well $(76 \%)$. Over- all, lactate excluding metabolic acidosis was a slightly better marker than $\mathrm{pH}$, $\mathrm{BD}$, and $(\mathrm{pH}+\mathrm{BD})$. If one aims to detect fetal metabolic acidosis severe enough to require immediate intervention, the sensitivity of the lactate cut-off needs to be high in order not to miss fetuses with metabolic acidosis.

As a marker of severe fetal metabolic acidosis, our study shows that lactate $\geq 6.6$ $\mathrm{mmol} / \mathrm{l}$ and a $\mathrm{BD} \geq 10 \mathrm{mmol} / \mathrm{l}$ have greater sensitivity than the traditionally used $\mathrm{pH} \leq$ 7.20 (Table 5). Overall, lactate to detect metabolic acidosis was a better marker than $\mathrm{pH}, \mathrm{BD}$, and $(\mathrm{pH}+\mathrm{BD})$. Lactate seemed to precede the fall in $\mathrm{pH}$, especially when the time interval between sampling and delivery exceeded 60 minutes (Table 6). From animal studies in the ovine fetus, it is known that after repetitive hypoxic insults, $\mathrm{pH}$ is restored to normal within minutes of recovery, whereas lactate remains increased for hours (29). This suggests that lactate, as a marker for fetal status, is less sensitive to the sampling-to-delivery interval than are $\mathrm{pH}$ and $\mathrm{BD}$.

As both the lactate concentration and $\mathrm{BD}$ are indicative of metabolic acidosis, a strong correlation between the two variables would be expected. A previous study in 2554 single- ton babies reported a strong correlation between cord blood lactate and $\mathrm{BD}(\mathrm{r}=0.83)$ (10). In our study, the correlation between lactate and $\mathrm{BD}$ in fetal scalp and cord blood was significant, but smaller than expected ( $r=0.48$ and $r=0.56$, respectively). Several factors may have contributed to this:

1) Our data reflect a population of fetuses at risk for metabolic acidosis, whereas the study by Gjerris also included cord blood data from uncomplicated deliveries (10). 
2) The weaker correlation between lactate and BD in FBS compared to cord blood in our study may be explained in part by FBS sampling errors, including mixture with tissue fluid or air, which may have affected the calculated BD. The chance of admixture was present in our study, especially because FBS required $35 \mu$ and median sampling time was five minutes.

3) Calculated $\mathrm{BD}$ values were not corrected for fetal hemoglobin or temperature, even in the case of maternal fever.

4) Lactate values in FBS represent an admixture of lactate values from various blood compartments, the concentration being highest in plasma, lower in hemolyzed blood, and lowest in whole blood with intact erythrocytes (30).

5) Lactate values in FBS may represent an admixture with extracellular and even amniotic fluid; amniotic fluid is rich in lactate, also in the absence of fetal acidosis. The chance of contamination is likely to be smaller when small sample volumes are needed and sampling time is short, as is the case if only lactate is measured (9).

In conclusion, the lactate concentration in fetal scalp blood correlates with both $\mathrm{pH}$ and $\mathrm{BD}$. The test characteristics that provide either reassurance or indication for immediate delivery seem to be better for lactate than for $\mathrm{pH}, \mathrm{BD}$, or the latter two combined. As lactate analysis is easier, faster, and more often successful than full blood gas analysis, fetal scalp blood lactate is an attractive alternative to full blood gas analysis during labor. 


\section{References}

(1) Low JA, Victory R, Derrick EJ. Predictive value of electronic fetal monitoring for intrapartum fetal asphyxia with metabolic acidosis. Obstet Gynecol 1999 Feb;93(2):285-91.

(2) Bretscher J, Saling E. pH values in the human fetus during labor. Am J Obstet Gynecol 1967 Apr 1;97(7):906-11.

(3) Low JA, Panagiotopoulos C, Derrick EJ. Newborn complications after intrapartum asphyxia with metabolic acidosis in the term fetus. Am J Obstet Gynecol 1994 Apr;170(4):1081-7.

(4) Low JA, Lindsay BG, Derrick EJ. Threshold of metabolic acidosis associated with newborn complications. Am J Obstet Gynecol 1997 Dec;177(6):1391-4.

(5) Siggaard-Andersen O. An acid-base chart for arterial blood with normal and pathophysiological reference areas. Scand J Clin Lab Invest 1971 May;27(3):239-45.

(6) Tuffnell D, Haw WL, Wilkinson K. How long does a fetal scalp blood sample take? BJOG 2006 Mar;113(3):332-4.

(7) Westgren M, Kruger K, Ek S, Grunevald C, Kublickas M, Naka K, et al. Lactate compared with $\mathrm{pH}$ analysis at fetal scalp blood sampling: a prospective randomised study. Br J Obstet Gynaecol 1998 Jan;105(1):29-33.

(8) Allen RM, Bowling FG, Oats JJ. Determining the fetal scalp lactate level that indicates the need for intervention in labor. Aust N Z J Obstet Gynaecol 2004 Dec;44(6):54952.

(9) Nordstrom L. Fetal scalp and cord blood lactate. Best Pract Res Clin Obstet Gynaecol 2004 Jun;18(3):467-76.

(10) Gjerris AC, Staer-Jensen J, Jorgensen JS, Bergholt T, Nickelsen C. Umbilical cord blood lactate: a valuable tool in the assessment of fetal metabolic acidosis. Eur J Obstet Gynecol Reprod Biol 2008 Jul;139(1):16-20.

(11) Kruger K, Kublickas M, Westgren M. Lactate in scalp and cord blood from fetuses with ominous fetal heart rate patterns. Obstet Gynecol 1998 Dec;92(6):918-22.

(12) Kruger K, Hallberg B, Blennow M, Kublickas M, Westgren M. Predictive value of fetal scalp blood lactate concentration and $\mathrm{pH}$ as markers of neurologic disability. Am J Obstet Gynecol 1999 Nov;181(5 Pt 1):1072-8.

(13) yres-de-Campos D, Bernardes J. Twenty-five years after the FIGO guidelines for the use of fetal monitoring: time for a simplified approach? Int J Gynaecol Obstet 2010 Jul;110(1):1-6.

(14) Wiberg N, Kallen K, Olofsson P. Base deficit estimation in umbilical cord blood is influenced by gestational age, choice of fetal fluid compartment, and algorithm for calculation. Am J Obstet Gynecol 2006 Dec;195(6):1651-6.

(15) Herbst A, Thorngren-Jerneck K, Wu L, Ingemarsson I. Different types of acid-base changes at birth, fetal heart rate patterns, and infant outcome at 4 years of age. Acta Obstet Gynecol Scand 1997 Nov;76(10):953-8. 
(16) Huch A, Huch R, Rooth G. Guidelines for blood sampling and measurement of $\mathrm{pH}$ and blood gas values in obstetrics. Based upon a workshop held in Zurich, Switzerland, March 19, 1993 by an Ad Hoc Committee. Eur J Obstet Gynecol Reprod Biol 1994 May 18;54(3):165-75.

(17) Wiberg-Itzel E, Lipponer C, Norman M, Herbst A, Prebensen D, Hansson A, et al. Determination of $\mathrm{pH}$ or lactate in fetal scalp blood in management of intrapartum fetal distress: randomised controlled multicentre trial. BMJ 2008 Jun 7;336(7656):1284-7.

(18) Goldaber KG, Gilstrap LC, III, Leveno KJ, Dax JS, McIntire DD. Pathologic fetal acidemia. Obstet Gynecol 1991 Dec;78(6):1103-7.

(19) Sehdev HM, Stamilio DM, Macones GA, Graham E, Morgan MA. Predictive factors for neonatal morbidity in neonates with an umbilical arterial cord $\mathrm{pH}$ less than 7.00. Am J Obstet Gynecol 1997 Nov;177(5):1030-4.

(20) Roemer VM. [Measured quantities in perinatal medicine--the base excess]. Z Geburtshilfe Neonatol 2005 Jun;209(3):81-9.

(21) Kastendieck E, Paulick R, Martius J. Lactate in fetal tissue during hypoxia; correlation to lactate, $\mathrm{pH}$ and base deficit in the fetal blood. Eur J Obstet Gynecol Reprod Biol 1988 Sep;29(1):61-71.

(22) Nordstrom L, Ingemarsson I, Westgren M. Fetal monitoring with lactate. Baillieres Clin Obstet Gynaecol 1996 Jun;10(2):225-42.

(23) East CE, Leader LR, Sheehan P, Henshall NE, Colditz PB. Intrapartum fetal scalp lactate sampling for fetal assessment in the presence of a non-reassuring fetal heart rate trace. Cochrane Database Syst Rev 2010;3:CD006174.

(24) Wiberg N, Kallen K, Herbst A, Olofsson P. Relation between umbilical cord blood $\mathrm{pH}$, base deficit, lactate, 5-minute Apgar score and development of hypoxic ischemic encephalopathy. Acta Obstet Gynecol Scand 2010 Oct;89(10):1263-9.

(25) Ball RH, Espinoza MI, Parer JT, Alon E, Vertommen J, Johnson J. Regional blood flow in asphyxiated fetuses with seizures. Am J Obstet Gynecol 1994 Jan;170(1 Pt 1):156-61.

(26) Ross MG, Gala R. Use of umbilical artery base excess: algorithm for the timing of hypoxic injury. Am J Obstet Gynecol 2002 Jul;187(1):1-9.

(27) Westerhuis ME, Strasser SM, Moons KG, Mol BW, Visser GH, Kwee A. [Intrapartum foetal monitoring: from stethoscope to ST analysis of the ECG]. Ned Tijdschr Geneeskd 2009;153.

(28) Holzmann M, Nordstrom L. Follow-up national survey (Sweden) of routines for intrapartum fetal surveillance. Acta Obstet Gynecol Scand 2010 May;89(5):712-4.

(29) Kaneko M, White S, Homan J, Richardson B. Cerebral blood flow and metabolism in relation to electrocortical activity with severe umbilical cord occlusion in the near-term ovine fetus. Am J Obstet Gynecol 2003 Apr;188(4):961-72. 
(30) Foxdal P, Sjodin B, Rudstam H, Ostman C, Ostman B, Hedenstierna GC. Lactate concentration differences in plasma, whole blood, capillary finger blood and erythrocytes during submaximal graded exercise in humans. Eur J Appl Physiol Occup Physiol 1990;61(3-4):218-22. 



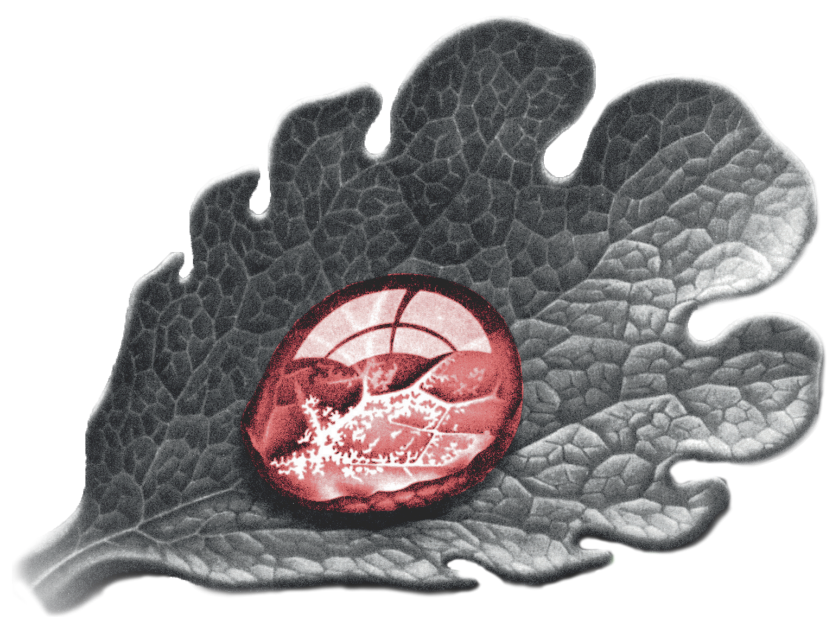




\section{Chapter 4}

COMPARISON OF TWO POINT-OF-CARE TESTING DEVICES FOR FETAL LACTATE DURING LABOR

Ayesha M.F. Heinis, Jacqueline Dinnissen, Marc E.A. Spaanderman, Fred K. Lotgering, Jacqueline M.T. Klein Gunnewiek 


\section{Abstract}

Background: Point-of-Care (POC) lactate testing of fetal scalp blood lactate is used as an alternative for $\mathrm{pH}$ analysis. Lactate measurements have not been standardized and values vary with each device used. The aim of this study was to evaluate the performance of two POC lactate meters for intrapartum use.

Methods: Analytical performance of StatStrip Lactate (Nova Biomedical) and Lactate Pro (Arkray) was evaluated using CLSI EP10. Both POC meters were compared with our lactate reference method (RapidLab 860; Siemens Healthcare Diagnostics) using fetal scalp and neonatal cord blood. Deming regression analysis was performed.

Results: StatStrip Lactate coefficients of variation (CVs) were 5.1\%, 5.0\%, and $2.6 \%$ at $0.9,7.5$, and $14.1 \mathrm{mmol} / \mathrm{L}$ lactate, respectively. CVs for Lactate Pro were $10.7 \%, 5.2 \%$, and $5.7 \%$ at $1.7,4.1$, and $6.4 \mathrm{mmol} / \mathrm{L}$ lactate, respectively. Consecutive lactate measurements in 37 fetal scalp and 122 cord blood samples revealed different test characteristics for the two POC devices. In fetal scalp blood: StatStrip Lactate $=1.13^{*}$ RapidLab $-0.39\left(\mathrm{R}^{2}=0.907\right)$, and Lactate Pro $=0.95^{*}$ RapidLab $-0.03\left(\mathrm{R}^{2}=0.823\right)$. In cord artery blood: StatStrip Lactate $=1.08^{*}$ RapidLab $-0.09\left(\mathrm{R}^{2}=0.810\right)$, and Lactate Pro $=$ $0.72 *$ RapidLab $+0.59\left(R^{2}=0.807\right)$.

Conclusions: Overall performance of both Lactate Pro and StatStrip Lactate was good, with StatStrip Lactate having smallest CVs and closest correlation to our reference method. Both StatStrip Lactate and Lactate Pro can be used as a lactate POC device for obstetric use. 


\section{Introduction}

In case of a non-reassuring or abnormal fetal heart rate pattern during labor, fetal blood scalp sampling (FBS) with $\mathrm{pH}$ analysis can be used to diagnose fetal hypoxia/ acidosis. FBS is associated with technical and practical problems. It is often difficult to obtain an adequate amount of blood for determination of $\mathrm{pH}$ or, preferably, full blood gas analysis. Modern apparatus still require 25 to $30 \mu \mathrm{L}$ of blood for $\mathrm{pH}$ analysis and a minimum of $50 \mu \mathrm{L}$ for a complete acid-base status. Air bubbles and inhomogeneous samples may jeopardize analysis. Furthermore, FBS with $\mathrm{pH}$ or complete acid-base status is time-consuming because of logistics of sampling and analysis (15-30 minutes) and has high failure rates (11-20\%) (1-3). If only $\mathrm{pH}$ is measured, interpretation of the results may be difficult. A transient fall in $\mathrm{pH}$ may occur during normal labor as a result of temporary $\mathrm{CO}_{2}$ accumulation in the absence of metabolic acidosis or considerable neonatal morbidity. Persistent severe hypoxia/ ischemia leads to lactic acid accumulation, which is the major contributor to an increased base deficit (BD). This metabolic component of fetal acidemia is the most important indicator of subsequent neonatal morbidity (4-6).

Lactate measurement in fetal scalp blood may be an attractive alternative for $\mathrm{pH} /$ full blood gas analysis in FBS. It requires only a tiny amount of blood (0.7-5.0 $\mu \mathrm{l})$, failure rates are low (1.3-2.9\%), and results of Point-of-Care testing (POC) devices are quickly available (13-60 seconds) (7). However, lactate measurements have not been standardized and values vary with the device used and the type of blood compartment sampled from (8-12) Therefore, test characteristics and lactate cut-off values in fetal scalp and neonatal cord blood have to be determined for each lactate measurement device.

The objective of this study was to compare the performance of POC lactate meters when applied to both fetal scalp and cord artery blood, using RapidLab as our lactate reference method. 


\section{Materials and methods}

\section{Sample collection}

The study was conducted with FBS and cord blood data obtained from women during labor at the Radboud University Nijmegen Medical Centre between December 2008 and June 2009. The Radboud University Medical Centre is a tertiary referral centre with approximately 1500 low, medium and high-risk deliveries per year. Deliveries are managed by residents or midwives supervised by obstetricians. Cardiotocograms are classified and interpreted according to the guidelines of the International Federation of Gynecology and Obstetrics (13). FBS is performed on indication by the obstetric caregiver in case of intermediary or abnormal cardiotocography trace. The frequency of FBS in our institution is around $11 \%(14)$.

The technique of FBS was carried out as previously reported (15). After the incision in the fetal scalp, blood was collected in a $125 \mathrm{ml}$ heparinized capillary tube (OmniLabo, Breda, the Netherlands), and send to the central laboratory for immediate analysis.

To systematically evaluate neonatal condition, umbilical cord blood gas analysis from both artery and vein is performed routinely. The cord is double clamped immediately after birth and punctured with a 19-gauge needle attached to a $125 \mu \mathrm{l}$ heparinized capillary tube. After removal of the needle, the samples are closed and sent to the central laboratory for immediate analysis of full blood gas and lactate. For the purpose of this study paired umbilical blood samples from 145 consecutive deliveries were obtained during a three-month period. Only data from the umbilical artery were used in the analysis, assuming it to reflect fetal condition best. Umbilical vein results were used to verify correct sampling. To exclude mix up of samples or samples both coming from the same vessel, only sample pairs were used in which arterial $\mathrm{pH}$ was at least 0.02 lower than venous $\mathrm{pH}$ (16).

Approval from the University Ethics Committee was sought but was waved due to the fact that this study was solely and anonymously performed to test the performance of the lactate meters. Lactate values were not available for clinical management.

\section{Measuring devices}

Because of the limited amount of fetal scalp blood, two POC lactate testing devices, potential candidates for use in our institution, were selected for evaluation. The two POC lactate testing devices evaluated in this study were the StatStrip Lactate (Nova Biomedical, Waltham, MA, USA) and the Lactate Pro (Arkray, Kyoto, Japan). The 
blood gas analyzer RapidLab 860 (Siemens Healthcare Diagnostics, Deerfield, IL, USA) was used to perform full blood gas analysis and served as a lactate reference method. The RapidLab 860 uses a lactate biosensor containing lactate oxidase. Analysis of lactate and full blood gas requires a minimum of $65 \mu \mathrm{L}$ The StatStrip Lactate and Lactate Pro are hand-held single-use electrochemical test strips devices. Both meters utilize an amperometric method using an enzymatic reaction with lactate oxidase and potassium ferricyanide as an electron mediator. Sampling volume for Lactate Pro is $5.0 \mathrm{~mL}$, while StatStrip Lactate uses $0.7 \mathrm{~mL}$.

\section{Analytical performance}

To get an overall impression of performance of the Lactate Pro and StatStrip Lactate, lactate concentrations were determined at three different levels according CLSI EP10. Commercial controls, Rapid QC (Siemens Healthcare Diagnostics, Deerfield, IL, USA), were used. Total coefficients of variation were determined based on withinday and between-day variation. Analytical performance was evaluated further using cord artery and fetal scalp blood in a comparison study.

\section{Measurements in fetal scalp and cord artery blood}

Three sequential lactate measurements were performed on Lactate Pro, StatStrip Lactate and RapidLab 860 as soon as the cord and fetal scalp blood samples arrived in the laboratory. The sequence was the same for all samples analyzed in this study. On the RapidLab 860 a full blood gas analysis was concomitantly performed. The results of sequential lactate measurements in both cord artery and FBS samples were compared as follows: RapidLab 860 versus i) Lactate Pro and ii) StatStrip Lactate. Regression was determined between: i) Lactate (RapidLab) and pH; ii) Lactate Pro and $\mathrm{pH}$; iii) StatStrip Lactate and $\mathrm{pH}$; iv) Lactate (RapidLab) and BD; v) Lactate Pro and BD; vi) StatStrip Lactate and BD.

\section{Statistics}

Correlation coefficients $\left(\mathrm{R}^{2}\right)$ between the different methods were determined using Deming regression analysis. Student's $t$ test for paired samples, two tails, was used to compare paired differences. A $p$-value $<0.05$ was considered significant.

Computations were performed using EP Evaluator ${ }^{\mathrm{TM}}$ 8.0 (D.G. Rhoads Associates Inc., South Burlington, USA) and SPSS 15.0 (SPSS Inc, Chicago, USA). 


\section{Results}

\section{Analytical performance of POC devices}

Performing CLSI EP10 at three different lactate levels yielded different mean lactate values. For RapidLab mean values were 0.9, 2.7 and $11.0 \mathrm{mmol} / \mathrm{L}$. For StatStrip Lactate mean values were $0.9,7.5$, and $14.1 \mathrm{mmol} / \mathrm{L}$, while for Lactate Pro mean values were $1.7,4.1$, and $6.4 \mathrm{mmol} / \mathrm{L}$. Total coefficients of variation $(\mathrm{CVs})$ for RapidLab were $5.5 \%, 2.7 \%$ and $5.2 \%$ at $0.9,2.7$ and $11.0 \mathrm{mmol} / \mathrm{L}$, respectively. CVs for StatStrip Lactate were 5.1\%, 5.0\%, and $2.6 \%$ at $0.9,7.5$, and $14.1 \mathrm{mmol} / \mathrm{L}$ lactate, respectively. CVs for Lactate Pro were $10.7 \%, 5.2 \%$, and $5.7 \%$ at 1.7, 4.1, and $6.4 \mathrm{mmol} / \mathrm{L}$ lactate, respectively.

\section{Results in fetal scalp blood}

During the study period 33 women underwent FBS during labor. In 6 women FBS was performed twice. In 1 woman FBS was performed three times. Intra-partum characteristics of 33 mother and baby pairs were as follows: average maternal age was 32.7 years; 17 women $(52 \%)$ were nulliparous and $16(48 \%)$ were parous; 32 women (97\%) had a singleton and $1(3 \%)$ had a twin pregnancy. Gestational age ranged from 37.0 till 42.3 weeks. Spontaneous onset of labor occurred in 17 women (42\%) and labor was induced in 16 women (48\%). Spontaneous vaginal delivery occurred in 14 women (42\%), 17 (52\%) had vacuum-extraction, and cesarean section due to dystocia was performed in 2 women (6\%). All neonates had an Apgarscore after 5 minutes of 8 or higher. Birth weight ranged from 2560 till $4608 \mathrm{~g}$. No complications due to the FBS procedure were reported. In total $40 \mathrm{FBS}$ samples were obtained. Lactate levels ranged from 1.6 till $6.7 \mathrm{mmol} / \mathrm{L}$ using RapidLab. Two results by StatStrip Lactate and 1 by Lactate Pro were considered invalid due to insufficient sample volume. These and their corresponding samples for the other meters were removed from further calculations. The tests results from the two POC devices and the reference method (RapidLab 860) were strongly correlated. Coefficients $\left(R^{2}\right)$ for StatStrip Lactate and Lactate Pro were 0.907 and 0.823, respectively (Figures 1-4). Results from Deming regression analysis are shown in Figures 1 and 2. For Lactate Pro (Figure 1): slope $=0.95(95 \% \mathrm{CI}$ ranging from 0.70 to 1.20$)$ and intercept $=$ -0.03 (95\% CI ranging from -0.81 to 0.75 ). For StatStrip Lactate (Figure 2): slope $=1.13(95 \% \mathrm{CI}$ ranging from 0.99 to 1.26$)$ and intercept $=0.39(95 \% \mathrm{CI}$ ranging from -0.85 to 0.07$)$. Student's $t$ analysis between paired samples showed a significant difference between RapidLab and Lactate Pro $(p=0.032)$ and a non-significant difference between RapidLab and StatStrip Lactate $(p=0.453)$. 


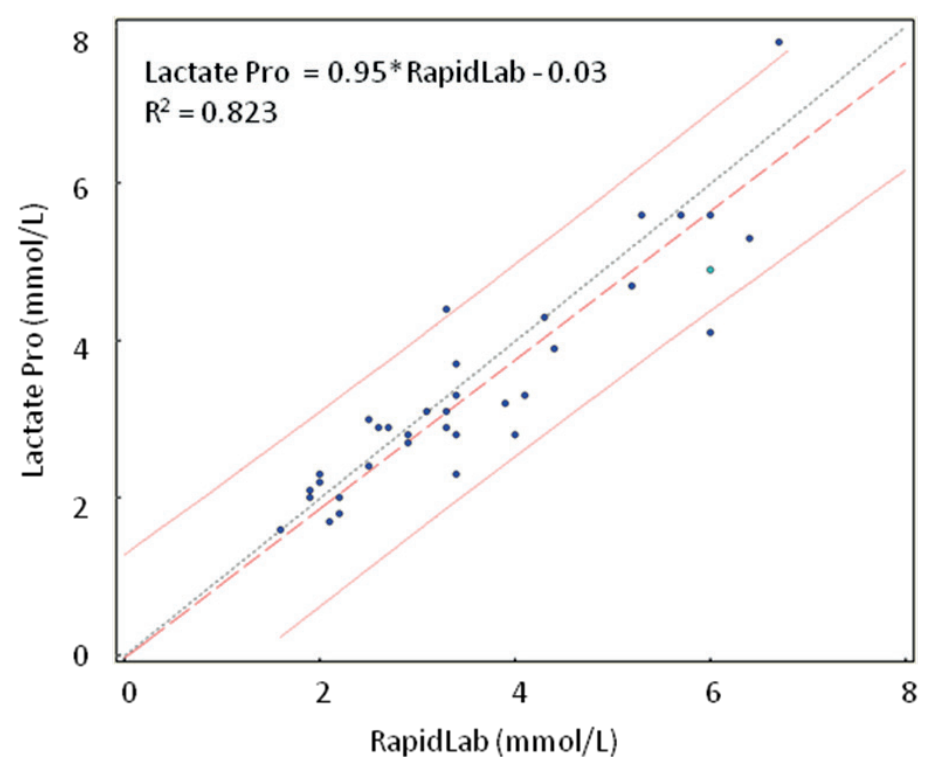

Figure 1. Deming regression lines in fetal scalp blood for RapidLab 860 (X-axis) versus Lactate Pro. Regression and correlation $\left(\mathrm{R}^{2}\right)$ are depicted. Lines of identity and regression are indicated. Dashed lines indicate $\pm 2 \mathrm{SD}$.

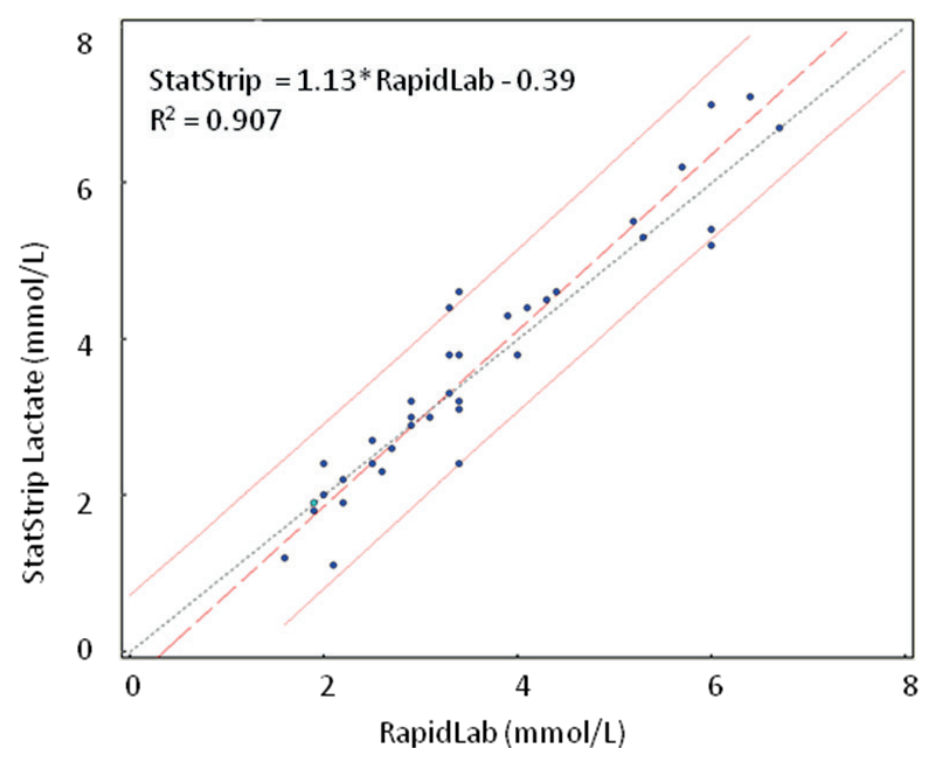

Figure 2. Deming regression lines in fetal scalp blood for RapidLab 860 (X-axis) versus StatStrip Lactate. Regression and correlation $\left(\mathrm{R}^{2}\right)$ are depicted. Lines of identity and regression are indicated. Dashed lines indicate $\pm 2 \mathrm{SD}$. 


\section{Results in cord artery blood}

During the study period paired umbilical artery and vein samples were obtained from 145 consecutive deliveries. After $\mathrm{pH}$ validation 20 paired samples were excluded (16). A further three samples were removed due to inadequate sample volume. After exclusions, 122 samples were available for analysis. The intra-partum characteristics in this group were as follows: average maternal age was 30.5 years; 60 women (49\%) were nulliparous and $62(51 \%)$ were parous; 116 women $(95 \%)$ had a singleton and $6(5 \%)$ a twin pregnancy. Gestational age ranged from 25.3 till 42.0 weeks. Spontaneous onset of labor occurred in 54 women (44\%), labor was induced in 56 women (46\%) and a planned cesarean section was performed in $12(10 \%)$. Spontaneous vaginal delivery occurred in $85(70 \%)$ women, $14(11 \%)$ had a vacuum-extraction and cesarean section due to dystocia was performed in 9 women $(7 \%)$. Two neonates $(2 \%)$ were delivered by primary breech extraction and $118(96 \%)$ had an Apgarscore after 5 minutes $\geq 8$. Four neonates (3\%) had an Apgarscore after 5 minutes $\leq 7$. Birth weight ranged from 777 till 4750 g. Lactate levels ranged from 1.6 till $14.5 \mathrm{mmol} / \mathrm{L}$ using RapidLab. Both StatStrip Lactate and Lactate Pro correlated strongly with the reference method (RapidLab 860), with coefficients $\left(\mathrm{R}^{2}\right)$ of 0.810 and 0.807 respectively (Figures 3 and 4). Results from Deming regression analysis are shown in Figures 3 and 4. For Lactate Pro (Figure 3): slope $=0.72(95 \% \mathrm{CI}$ ranging from 0.61 to 0.83$)$ and intercept $=0.59(95 \% \mathrm{CI}$ ranging from

0.12 to 1.06 ). For StatStrip Lactate (Figure 4): slope $=1.08$ (95\% CI ranging from 0.91 to 1.26$)$ and intercept $=-0.09$ (95\% CI ranging from -0.81 to 0.62$)$. Student's $t$ analysis between paired samples showed a significant difference between RapidLab and Lactate Pro $(p<0.001)$ as well as between RapidLab and StatStrip Lactate ( $p=$ $0.001)$. 


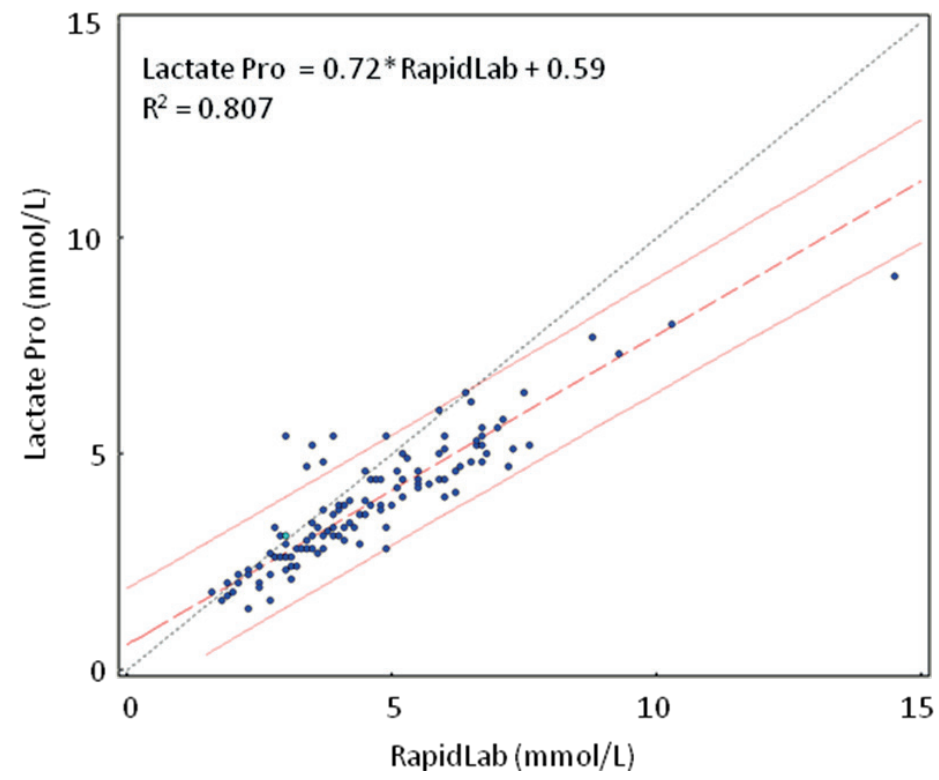

Figure 3. Deming regression lines in cord artery blood for RapidLab 860 (X-axis) versus Lactate Pro. Regression and correlation $\left(\mathrm{R}^{2}\right)$ are depicted. Lines of identity and regression are indicated. Dashed lines indicate \pm 2 SD.

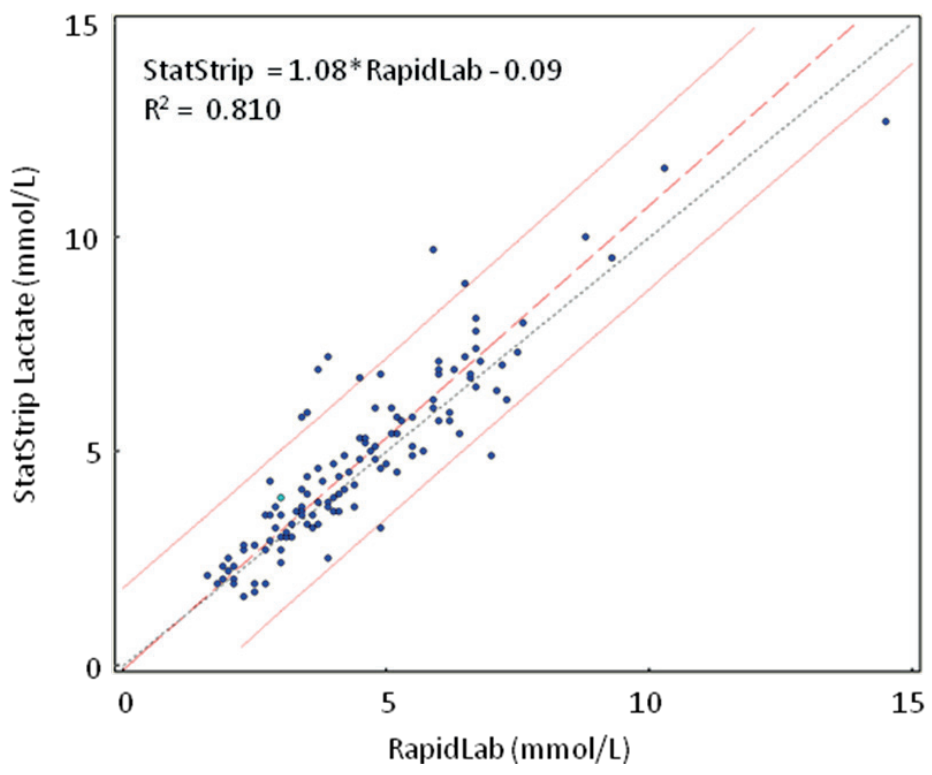

Figure 4. Deming regression lines in cord artery blood for RapidLab 860 (X-axis) versus StatStrip Lactate. Regression and correlation $\left(\mathrm{R}^{2}\right)$ are depicted. Lines of identity and regression are indicated. Dashed lines indicate $\pm 2 \mathrm{SD}$. 


\section{Discussion}

POC lactate test strip meters seem attractive for obstetric use because of the small sample size and rapid generation of results. Previous studies have evaluated POC meters for fetal scalp blood lactate assessment during labor (12;17-20). However, except for one (18), these studies have used neonatal cord blood for evaluation of the devices, and not fetal scalp blood obtained during labor. This study has evaluated the performance of a lactate meter using fetal scalp blood as well as neonatal cord artery blood.

When testing analytical performance of Lactate Pro and StatStrip Lactate using commercial control material, we observed large differences (up to $7.7 \mathrm{mmol} / \mathrm{l}$ ) between absolute mean values at the three different concentration levels. These discrepancies might be caused by the fact that the matrix of these control materials is different from human blood. Analytical performance and coefficients of variation of the Lactate Pro was evaluated in a previous study using Maine standards at two different levels (12). In this study by Ridenour and co-workers' differences in mean values are also observed, although they were less profound compared to our results. Ridenour and colleagues also found smaller coefficients of variation for Lactate Pro compared to our study, namely $4,0 \%$ at low $(3.2 \mathrm{mmol} / \mathrm{L})$ and $3.2 \%$ at high $(10.4 \mathrm{mmol} / \mathrm{L})$ levels $(12)$. This might be caused by the fact that different control material was used compared to this study. These discrepancies show that commercial control material of non-human origin cannot be used to compare different POC lactate devices. Moreover, no gold standard for lactate measurement is available. This hampers interpretation of data. Standardization of lactate measurements is needed to eliminate these huge differences between different devices.

Deming regression analysis using fetal scalp blood showed good correlations between Lactate Pro as well as StatStrip Lactate and our reference method as shown in Figures 1 and 2. StatStrip Lactate yielded the best correlation. However, we observed differences in regression lines when using Lactate Pro and StatStrip Lactate. Both devices showed a slope and intercept in comparison with RapidLab 860. The slope and intercept were smallest when using Lactate Pro. However, Student's $t$ analysis of paired samples showed a significant difference between RapidLab and Lactate Pro $(p=0.032)$ while a non-significant difference between RapidLab and StatStrip Lactate was observed $(\mathrm{p}=0.453)$. When cord artery blood was used similar correlations between Lactate Pro, StatStrip Lactate and our reference method are obtained $\left(\mathrm{R}^{2}\right.$ $=0.807$ and 0.810 , respectively). The regression line of StatStrip Lactate from cord blood samples was comparable to the one found with fetal scalp blood (Figures 2 and 
4). However, the regression line of Lactate Pro in cord artery blood differed from fetal scalp blood (Figures 1 and 3): $y=0.72 x+0.59$ and $y=0.95 x-0.03$, respectively. Similar discrepancies were found by others. In the study by Ridenour and colleagues, in which lactate in cord artery blood is analyzed using Nova Lactate Plus and Lactate Pro, similar differences in regression lines were observed.(12) This phenomenon is also observed by Nordström and co-workers who also used two different devices, i.e. Lactate Pro and Accusport for measurements in cord blood (21). Reference material in order to standardize the different lactate methods (e.g lactate measurements in plasma, hemolyzed blood or whole blood with intact erythrocytes), is not present. This lack of standardization is the most probable explanation for the fact that different regression lines are observed when different lactate measuring devices are used.

Surprisingly, we also found different regression lines when blood from different sources (fetal scalp versus cord blood) were used. This phenomenon is more profound when using Lactate Pro. Obtaining blood from the umbilical cord after birth is much easier than deriving blood from the fetal scalp. Fetal scalp blood is a mixture of blood and a variable amount of tissue fluid. This might be an explanation for the observed differences.

In conclusion, this study shows that caution should be taken when interpreting results from lactate measurements obtained from different devices and different blood sources. We have shown that both StatStrip Lactate and Lactate Pro can be used as a POC device for lactate measurements in fetal scalp and cord artery blood. StatStrip Lactate showed the best test characteristics, with smallest CVs, the closest correlation to and the highest agreement with our reference method. No significant difference between lactate values in FBS measured with StatStrip Lactate and our reference method was found. 


\section{References}

(1) Tuffnell D, Haw WL, Wilkinson K. How long does a fetal scalp blood sample take? BJOG 2006;113:332-4.

(2) Westgren M, Kruger K, Ek S, Grunevald C, Kublickas M, Naka K, Wolff K, Persson B. Lactate compared with $\mathrm{pH}$ analysis at fetal scalp blood sampling: a prospective randomised study. Br J Obstet Gynaecol 1998;105:29-33.

(3) Ramanah R, Martin A, Clement M, Maillet R, Riethmuller D. Fetal scalp lactate microsampling for non-reassuring fetal status during labor: a prospective observational study. Fetal Diagn Ther 2010;27:14-9.

(4) Kruger K, Hallberg B, Blennow M, Kublickas M, Westgren M. Predictive value of fetal scalp blood lactate concentration and $\mathrm{pH}$ as markers of neurologic disability. Am J Obstet Gynecol 1999;181:1072-8.

(5) Low JA, Panagiotopoulos C, Derrick EJ. Newborn complications after intrapartum asphyxia with metabolic acidosis in the term fetus. Am J Obstet Gynecol 1994;170:10817.

(6) Low JA, Lindsay BG, Derrick EJ. Threshold of metabolic acidosis associated with newborn complications. Am J Obstet Gynecol 1997;177:1391-4.

(7) East CE, Leader LR, Sheehan P, Henshall NE, Colditz PB. Intrapartum fetal scalp lactate sampling for fetal assessment in the presence of a non-reassuring fetal heart rate trace. Cochrane Database Syst Rev 2010;3:CD006174.

(8) Boldt J, Kumle B, Suttner S, Haisch G. Point-of-care (POC) testing of lactate in the intensive care patient. Accuracy, reliability, and costs of different measurement systems. Acta Anaesthesiol Scand 2001;45:194-9.

(9) Brinkert W, Rommes JH, Bakker J. Lactate measurements in critically ill patients with a hand-held analyser. Intensive Care Med 1999;25:966-9.

(10) Foxdal P, Sjodin B, Rudstam H, Ostman C, Ostman B, Hedenstierna GC. Lactate concentration differences in plasma, whole blood, capillary finger blood and erythrocytes during submaximal graded exercise in humans. Eur J Appl Physiol Occup Physiol 1990;61:218-22.

(11) Karon BS, Scott R, Burritt MF, Santrach PJ. Comparison of lactate values between point-of-care and central laboratory analyzers. Am J Clin Pathol 2007;128:168-71.

(12) Ridenour RV, Gada RP, Brost BC, Karon BS. Comparison and validation of point of care lactate meters as a replacement for fetal $\mathrm{pH}$ measurement. Clin Biochem 2008;41:1461-5.

(13) Yres-de-Campos D, Bernardes J. Twenty-five years after the FIGO guidelines for the use of fetal monitoring: time for a simplified approach? Int J Gynaecol Obstet 2010;110:1-6.

(14) Heinis AM, Spaanderman ME, Klein Gunnewiek JM, Lotgering FK. Scalp blood lactate for intra partum assessment of fetal metabolic acidosis. Acta Obstet Gynecol Scand 2011;Jul 13 doi:10.1111/j.1600-0412.2011.01237.x. [Epub ahead of print]. 
(15) Saling E. [Technic For The Endoscopic Micro-Sampling Of Blood From The Fetus.]. Geburtshilfe Frauenheilkd 1964;24:464-9.

(16) Westgate J, Garibaldi JM, Greene KR. Umbilical cord blood gas analysis at delivery: a time for quality data. Br J Obstet Gynaecol 1994;101:1054-63.

(17) Nordstrom L, Chua S, Roy A, Arulkumaran S. Quality assessment of two lactate test strip methods suitable for obstetric use. J Perinat Med 1998;26:83-8.

(18) Nordstrom L, Persson B, Shimojo N, Westgren M. Fetal scalp and umbilical artery blood lactate measured with a new test strip method. Br J Obstet Gynaecol 1992;99:307-9.

(19) Pennell CE, Tracy MB. A new method for rapid measurement of lactate in fetal and neonatal blood. Austr NZ J Obstet Gynaecol 1999;39:227-33.

(20) Luttkus AK, Fotopoulou C, Sehouli J, Stupin J, Dudenhausen JW. Technical performance of lactate biosensors and a test-strip device during labor. Z Geburtshilfe Neonatol 2010;214:62-7.

(21) Nordstrom L. Lactate measurements in scalp and cord arterial blood. Curr Opin Obstet Gynecol 2001;13:141-5. 


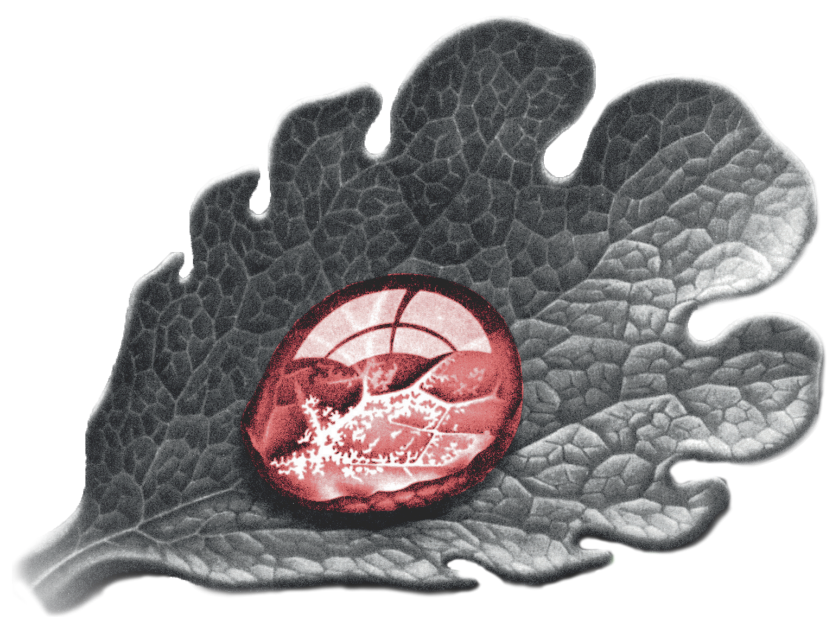




\section{Chapter 5}

CLINICAL EVALUATION OF STATSTRIP ${ }^{\circledR}$ LACTATE FOR USE IN FETAL SCALP BLOOD SAMPLING

Ayesha Heinis, Jeroen van Dillen, Janine Oosting, Sarah Rhöse,

Frank Vandenbussche, Joris Van Drongelen

Acta Obstetrica Gynecolica Scandinavica 2017; 96(3):334-341 


\section{Abstract}

Introduction: Point-of-Care testing of fetal scalp blood lactate is used as an alternative for $\mathrm{pH}$ analysis in fetal scalp blood sampling (FBS) during labor. Lactate measurements are not standardized and values vary with each device used. The aim of this study was to evaluate StatStrip Lactate (SSL) in the clinical setting in comparison to lactate (RLL) and $\mathrm{pH}(\mathrm{RLpH})$ by RapidLab.

Materials and methods: We obtained 323 FBS samples from 139 women. Parallel sampling of SSL and RLL/RLpH was performed in 247 samples. Outcome measures were the agreement and discrepancy rates between SSL, RLL and RLpH and the failure rate of all three methods. We constructed a Bland-Altman graph to assess the variability between the measurements across the range of values. The discrepancy rates between methods were compared using previously established cut-off values for SSL indicating reassurance $(<5.7 \mathrm{mmol} / \mathrm{L})$ and immediate delivery $(>7.0 \mathrm{mmol} / \mathrm{L})$ to those for $\mathrm{RLpH}$ $(<7.20$ and $>7.25)$

Results: SSL showed excellent agreement with RLL $\left(\mathrm{R}^{2}=0.742\right)$ and poor agreement with $\mathrm{RLpH}\left(\mathrm{R}^{2}=0.204\right)$. Failure rates for SSL, RLL and RLpH were $7 \%, 43 \%$ and $23 \%$ respectively. Using the cut-off values for reassurance and immediate delivery, the discrepancy rates between SSL and RLpH were $14 \%$ and $5 \%$ respectively.

Conclusions: SSL is a reliable test to measure lactate in FBS at a low failure rate. As there are discrepancies between SSL and RLpH and the cut-off values have not yet been evaluated prospectively regarding intervention rates and neonatal outcome, we recommend using SSL in addition to $\mathrm{pH}$ in FBS. 


\section{Introduction}

Fetal scalp blood sampling (FBS) is used in addition to a non-reassuring intra partum cardiotocogram (CTG) to assess fetal acidosis. FBS with $\mathrm{pH}$ analysis has been considered gold standard but has some major drawbacks; a relatively large amount of blood is needed ( 35 to $100 \mu \mathrm{L}$ ) and the logistics of sampling and analysis are time consuming (15-30 minutes) with high failure rates $(11-20 \%)(1,2)$. Moreover, handling of the sample, aerobic contamination and processing affect $\mathrm{pH}$ results. Lactate measurement in FBS is an attractive alternative. Persistent hypoxia/ischemia leads to lactate accumulation and metabolic acidosis, which is the most important indicator of subsequent neonatal morbidity $(3,4)$. Several studies, including randomized controlled trials have shown that scalp blood lactate has similar or better predictive properties in identifying short-term neonatal morbidity than fetal $\mathrm{pH}$, without affecting the rate of metabolic acidosis at birth or operative deliveries for suspected fetal distress (5-7).

Point of care testing (POC) for lactate has several advantages. It requires a tiny amount of blood $(0.7-5.0 \mu \mathrm{l})$ and results are rapidly available (13-60 seconds) at a low failure rate $(1-3 \%)(2,8,9)$. However, POC lactate measurements are not standardized and values vary with the device used and the type of blood compartment sampled from (i.e. plasma, hemolysed blood or whole blood with intact erythrocytes). Therefore, the test characteristics and cut-off values in fetal scalp blood have to be established for each device. In a previous study, we compared the characteristics of two POC lactate devices, Lactate Pro (Akray, Kyoto, Japan) and StatStrip Lactate (SSL), in a laboratory setting, using the Rapid Lab blood gas analyzer as a reference method. As compared to the Lactate Pro, SSL showed a closer correlation with the reference method, better test characteristics, and a smaller coefficient of variation (CV) (10). The value of SSL for its use in the clinical setting remained to be evaluated.

The aim of this study was to investigate SSL for its use in FBS in a clinical setting. For this purpose, we assessed the agreement between SSL, lactate (RLL) and pH (RLpH) measured on the RapidLab blood gas analyzer. We also assessed the discrepancy rates between the methods using the previously established SSL cut-off values that served as indicators for both reassurance and immediate delivery. 


\section{Materials and methods}

We conducted a prospective observational study with SSL in FBS obtained from women with suspected fetal distress during labor. The study was performed at the Radboud University Medical Center between 13 February 2010 and 12 March 2011. Included were women with a singleton fetus in vertex presentation and a gestational age of 34 weeks or over. The study was approved by the University Ethics Committee (2009/345).

The Radboud University Medical Center is a tertiary referral center with approximately 1500 deliveries per year (low, medium and high-risk). Deliveries are managed by midwives and residents supervised by obstetricians. Cardiotocograms (CTG's) are classified and interpreted according to the guidelines of the International Federation of Gynecology and Obstetrics (11). FBS is performed in case of an intermediary or abnormal CTG trace, as interpreted by the obstetric caregiver. The percentage of deliveries in which FBS is performed is approximately $11 \%(12)$.

FBS was carried out by standard technique (13). After incision of the fetal scalp, blood was collected in heparinized plastic capillaries. POC lactate was performed with the Statstrip Lactate (Nova Biomedical, Waltham, MA, USA), which is a handheld single-use device that utilizes $0.7 \mathrm{~mL}$ of blood and electrochemical test strips. After POCT lactate testing, the remaining sample was sent to the laboratory for immediate full blood gas and lactate analysis on a blood gas analyzer (RapidLab 860, Siemens Healthcare Diagnostics, Deerfield, IL, USA). This required samples of 50$100 \mu$ l. If sampling volume is insufficient at best only $\mathrm{pH}$ is measured. Base deficit was calculated for the blood compartment $[\mathrm{BD}(\mathrm{B})]$, using the algorithm: $\mathrm{BD}(\mathrm{B})=$ $\left.(1-0.014 \times c t \mathrm{Hb})\left[c \mathrm{HCO}_{3}{ }^{-}-24.8\right)+(1.43 \times c t \mathrm{Hb}+7.7)(\mathrm{pH}-7.40)\right]$. Clinical management was solely based on RLpH. Guidelines were as follows: $\mathrm{RLpH} \geq 7.25$ normal; continue fetal monitoring and $\mathrm{pH} \leq 7.20$ acidemia; consider delivery. $\mathrm{pH}$ value between 7.20 and 7.25 pre-acidemia; repeated sampling after 30 minutes. For the purpose of this study we considered a SSL value $\leq 5.7 \mathrm{mmol} / \mathrm{l}$ indicative for reassuring fetal status and a SSL value $\geq 7.0 \mathrm{mmol} / \mathrm{l}$ representing acidemia. These cut-off values were established by introducing the RL lactate cut-off values ( 5.4 and $6.6 \mathrm{mmol} / \mathrm{L}$ ) from a retrospective cohort into the formula: $\mathrm{SSL}=1.13^{*} \mathrm{RL}-0.39$, which was generated from a comparison study of RL and SSL in 38 FBS samples $(10,12)$. The discrepancy rates were studied using cross-tabulation. 
Neonatal acid-base status was evaluated systematically through umbilical cord blood gas analysis from both artery and vein immediately after birth. For the purpose of this study only data from the umbilical artery (UA) were used in the analysis, assuming it to reflect fetal condition best. Severe metabolic acidosis at birth was defined as $\mathrm{pH}$ $<7.05$ and $\mathrm{BD}>12.0 \mathrm{mmol} / \mathrm{l}$ in UA blood (7).

Statistical analysis was performed with SPSS version 22 (IBM SPSS Inc, Armonk, New York). Relations between SSL, RLL and RLpH in FBS were tested using regression analysis. We constructed a Bland-Altman graph to assess the variability between the measurements across the range of values for SSL and RLL. Data are presented as medians with 95 (2.5-97.5) centile ranges. 


\section{Results}

During the study period 1589 women delivered at the Radboud University Medical Center. FBS was performed in 139 (9\%) women. The intra-partum characteristics of these mother-baby pairs are summarized in Table 1 . There were no complications of the FBS procedure. There was a wide variance in the sampling to delivery interval, ranging from a few minutes till several hours. In $112(35 \%)$ cases FBS was performed at full dilatation. Three percent of infants in the studied population had metabolic acidosis at birth.

Table 1. Intra partum characteristics of 139 mother and baby pairs.

\begin{tabular}{lc}
\hline Characteristic & Number (\%) or median (range) \\
\hline Maternal age (years) & $30(19-41)$ \\
Nulliparous & $111(80 \%)$ \\
Gestational age (days) & $278(252-293)$ \\
Sampling to delivery interval (min) & $60(10-482)$ \\
Spontaneous vaginal delivery & $52(37 \%)$ \\
Ventouse delivery & $52(37 \%)$ \\
Cesarean section & $35(25 \%)$ \\
ODFD & $44(32 \%)$ \\
Birth weight (g) & $3383(2360-4375)$ \\
Five minute Apgar score $<7$ & $2(1 \%)$ \\
Metabolic acidosis at birth & $4(3 \%)$ \\
\hline
\end{tabular}

Data are numbers and percentage of total (\%), or medians with $2.5^{\text {th }}$ and $97.5^{\text {th }}$ centile. ${ }^{\text {a }}$ Operative delivery for fetal distress. ${ }^{\mathrm{b}} \mathrm{pH}<7.05$ and base deficit $\geq 12 \mathrm{mmol} / \mathrm{l}$ in umbilical artery blood.

From the 139 women, a total of 323 fetal scalp blood samples were obtained in which RLL and RLpH were measured (Figure 1). Parallel measurement of SSL was performed in 247 of these samples. SSL, RLL and RLpH analysis failed in 7\% (17/247), 23\% (73/323) and 43\% (140/323) respectively. For further analysis, one sample was excluded due to a 30-minute time interval between SSL and RLL/RLpH measurement. 


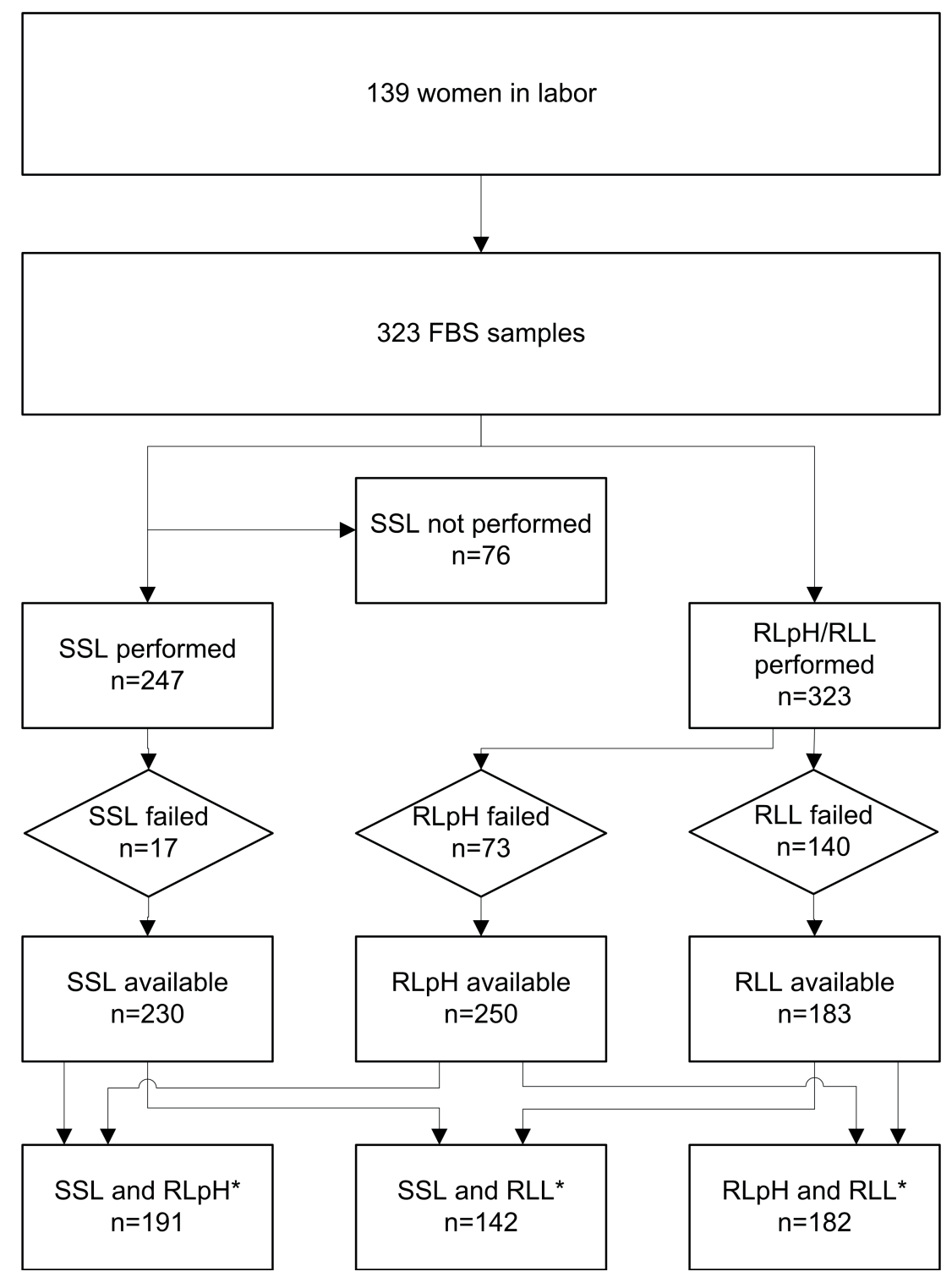

Figure 1. Flowchart of the study population. *One sample was excluded due to a 30 min interval between SSL and RLL/pH analysis. FBS, fetal scalp blood sampling; SSL, StatStrip ${ }^{\circledast}$ Lactate; RLpH, RapidLab ${ }^{\circledast}$ pH; RLL, RapidLab ${ }^{\circledast}$ Lactate. 
In 142 fetal scalp blood samples both SSL and RLL results were available. We found a strong agreement $\left(\mathrm{R}^{2}=0.747\right)$ as shown in Figure 2. In $82 \%(117 / 142)$ of the samples SSL $(\leq 5.7 \mathrm{mmol} / \mathrm{l})$ correctly indicated the absence of lactemia (RLL $\leq 5.4$ $\mathrm{mol} / \mathrm{L}$ ). There was a discrepancy between SSL and RLL for these cut-off values in $6 \%$ $(9 / 142)$ of the samples. In $5 \%$ (7/142) of the samples SSL $(\geq 7.0 \mathrm{mmol} / \mathrm{L})$ correctly indicated the presence of lactemia (RLL $\geq 6.6 \mathrm{mmol} / \mathrm{L}$ ). There was a discrepancy between SSL and RLL for these cut-off values in 2\% (3/142) of the samples. A Bland-Altman graph analysis showed minimal variance between SSL and RLL, and a slight linear increase with increasing lactate concentrations, i.e., SSL measured slightly higher than RLL with increasing lactate values (Figure 3).

In 191 fetal scalp blood samples both SSL and RLpH results were available. Fetal scalp SSL values correlated poorly with scalp RLpH (Figure 4, $\mathrm{R}^{2}=0.206$ ). In $77 \%$ $(148 / 191)$ of the samples SSL $(\leq 5.7 \mathrm{mmol} / \mathrm{l})$ correctly indicated the absence of acidemia ( $\mathrm{RLpH} \geq 7.25)$. There was a discrepancy between SSL and RLpH for these cut-off values in 14\% (27/191) of the samples. From these discrepant samples, 15 cases showed normal SSL values, whereas $\mathrm{pH}$ did not. In these cases, all but one $\mathrm{RLpH}$ value (7.08) indicated pre-acidemia (7.20-7.25). Twelve cases showed normal $\mathrm{RLpH}$ values, whereas SSL did not. Of these, SSL levels indicated pre-acidemia $(5.7-7.0 \mathrm{mmol} / \mathrm{L})$ and acidemia $(\geq 7.0 \mathrm{mmol} / \mathrm{L})$ in nine and three cases respectively.

In $3 \%(6 / 191)$ SSL $(\geq 7.0 \mathrm{mmol} / \mathrm{l})$ correctly indicated the presence of acidemia $(\mathrm{RLpH} \leq 7.20)$. There was a discrepancy between SSL and RLpH for these cutoff values in 5\% (10/191) of the samples. In Table 2 these discrepant samples are presented in more detail. Five cases showed SSL values indicating acidemia, whereas $\mathrm{RLpH}$ did not. In these cases, RLpH values were within the normal to pre-acidemic range. None of these infants had metabolic acidosis at birth. The five cases where $\mathrm{RLpH}$ indicated acidemia and SSL did not, SSL levels were all in the pre-acidemic range $(5.7-7.0 \mathrm{mmol} / \mathrm{l})$ except for case number nine. In this child, a scalp blood sample was taken within 26 minutes of birth. There was a large discrepancy between SSL $(3.8 \mathrm{mmol} / \mathrm{l})$ and RLpH (7.08) in this FBS sample. Further investigation of this case showed that a ventouse delivery was performed because of persistent bradycardia. In all cases SSL values were in line with the available RLL values, suggesting a correct lactate measurement rather than a false negative result in relation to RLpH. 


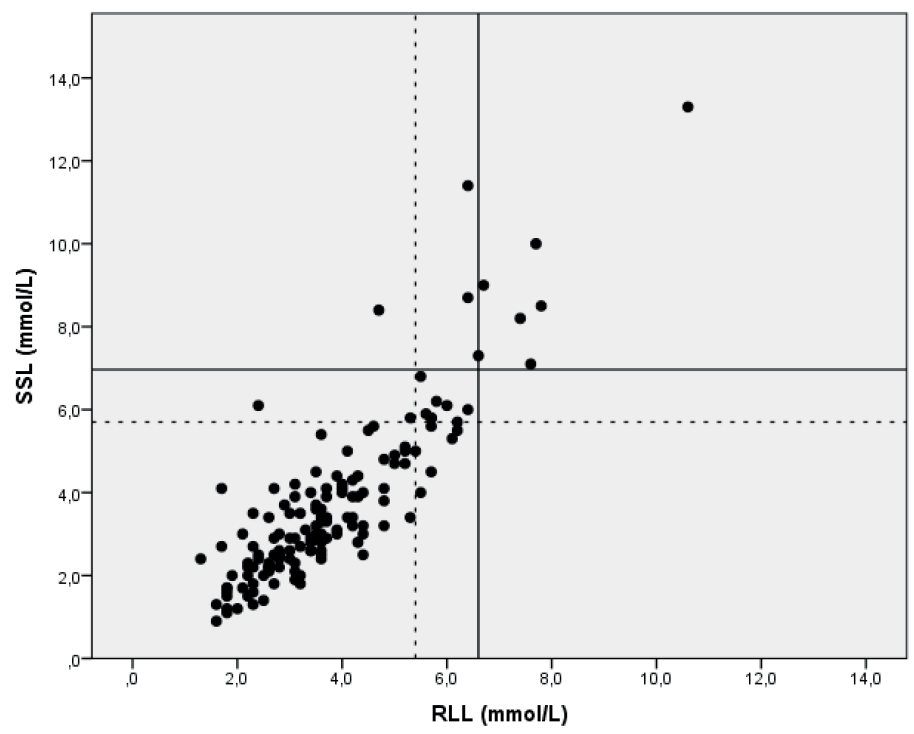

Figure 2. Scatterplot of 142 lactate determinations with Statstrip Lactate (SSL) and Rapidlab (RLL). Dashed lines correspond to cut-off values for reassurance (SSL $\leq$ $5.7 \mathrm{mmol} / \mathrm{L}$ and RLL $\leq 5.4 \mathrm{mmol} / \mathrm{L}$ ). Continuous lines correspond to cut-off values for immediate delivery (SSL $\geq 7.0 \mathrm{mmol} / \mathrm{L}$ and $\mathrm{RLL} \geq 6.6 \mathrm{mmol} / \mathrm{L}$ ).

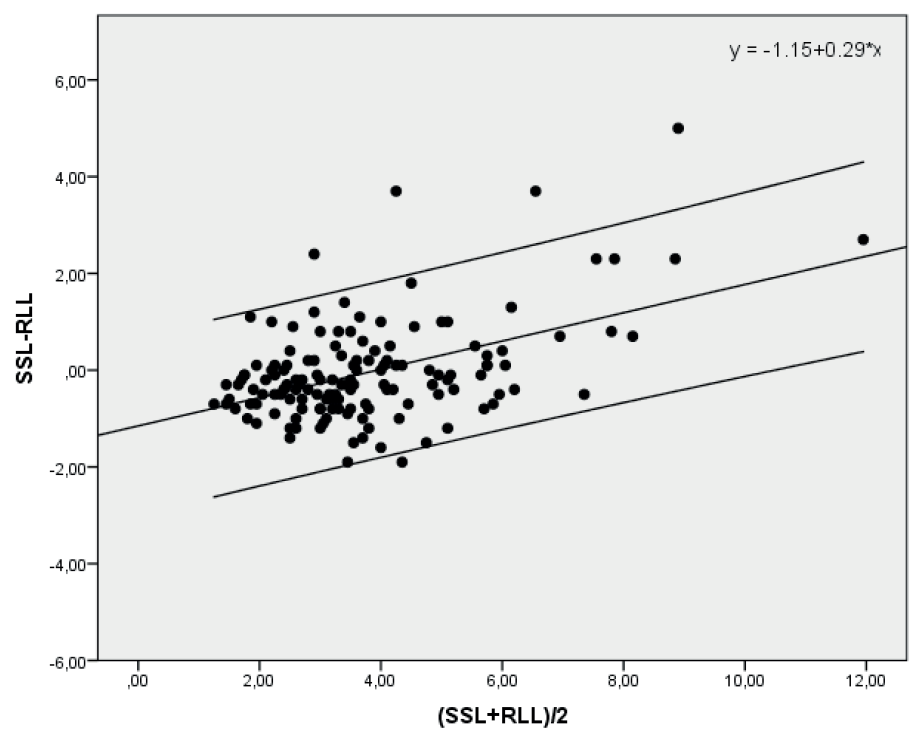

Figure 3. Bland-Altman plot with a regression line (including $95 \%$ prediction intervals) of differences between lactate concentrations (mmol/L) as measured by Statstrip Lactate (SSL) and RapidLab (RLL). Slope and intercept are indicated. 


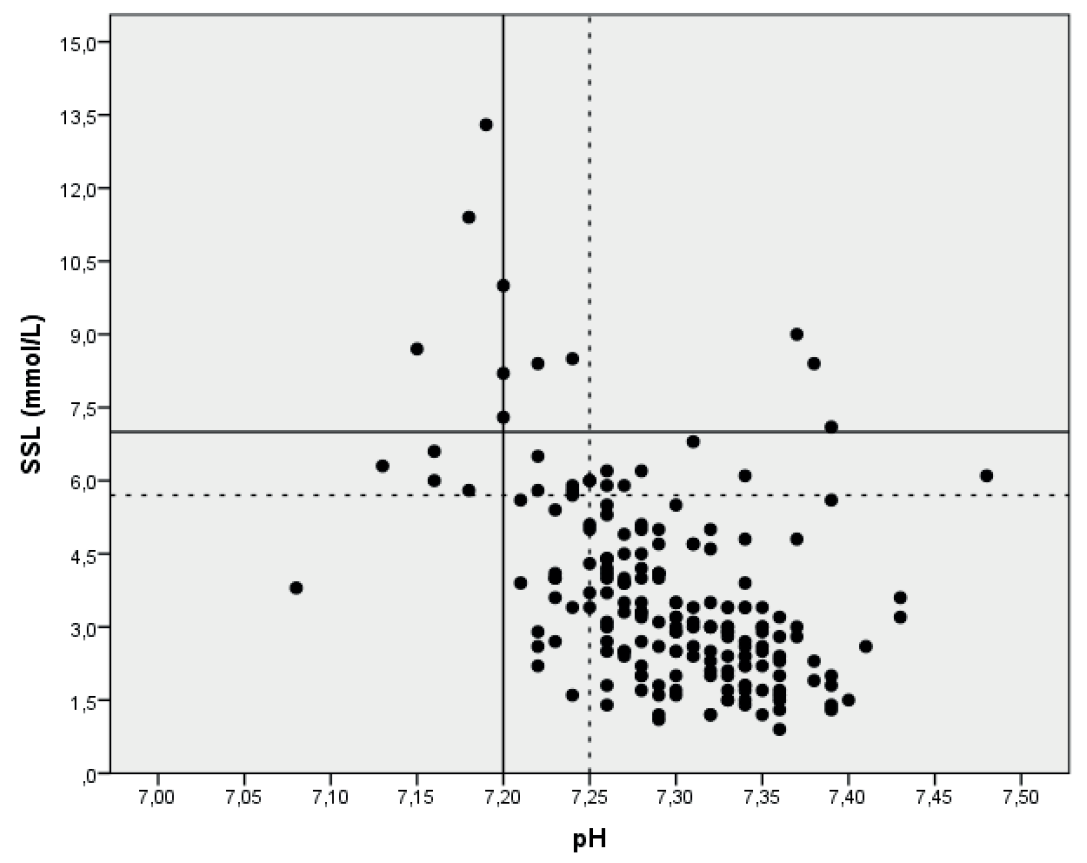

Figure 4. Scatterplot of 191 lactate determinations with Statstrip Lactate (SSL) and Rapidlab pH (RLpH). Dashed lines correspond to cut-off values for reassurance (SSL $\leq$ $5.7 \mathrm{mmol} / \mathrm{L}$ and $\mathrm{RLpH} \geq 7.25 \mathrm{mmol} / \mathrm{L}$ ). Continuous lines correspond to cut-off values for immediate delivery (SSL $\geq 7.0 \mathrm{mmol} / \mathrm{L}$ and $\mathrm{RLpH} \leq 7.20 \mathrm{mmol} / \mathrm{L}$ ).

In 182 fetal scalp blood samples both RLL and RLpH results were available. Fetal scalp RLL values correlated poorly with scalp $\mathrm{RLpH}\left(\mathrm{R}^{2}=0.110\right)$. We observed discrepancies between RLL $(\leq 5.4 \mathrm{mmol} / \mathrm{l})$ and RLpH $(\geq 7.25)$ in $17 \%(31 / 182)$ of the samples. For the cut-off values for RLL $(\geq 6.6 \mathrm{mmol} / \mathrm{L})$ and and $\mathrm{RLpH}(\leq 7.20)$ those were $7 \%(12 / 182)$. These numbers were comparable to the ones we observed between SSL and RLpH. 


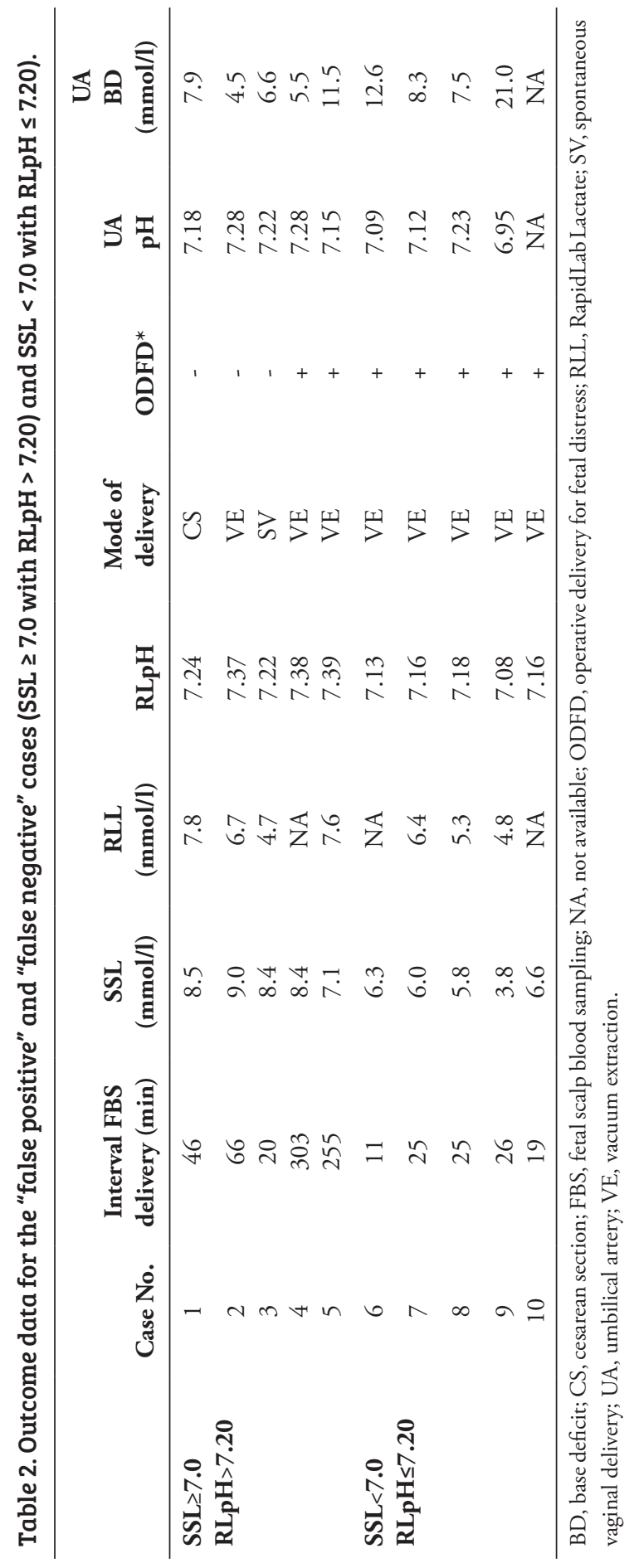




\section{Discussion}

In this study, we tested the value of SSL for its use in FBS in a clinical setting. We found that the lactate level in fetal scalp blood is accurately measured by SSL at a much lower failure rate compared to RLpH and RLL. In the vast majority of cases SSL cut-off values provided correct information on fetal status.

Previous studies that have validated SSL showed reliable results $(10,19,20)$. However, these studies only used cord blood, or a small number of fetal scalp samples and therefore do not adequately represent real labor ward conditions. To our best knowledge this is the first study that has evaluated a POC lactate device in a clinical setting with a large number of scalp blood samples. We found a strong agreement of SSL in comparison to RLL. This suggests that SSL is suitable for POC lactate analysis in FBS.

Measurement of SSL has a lower failure rate than RLpH/RLL. We detected a SSL failure rate of $7 \%$. This is fairly high compared to literature $(1-3 \%)(2,6,8)$. This might be due to the fact that our labor ward staff was not yet familiar with the device and that samples were not immediately repeated after failure. Recent experience with SSL on our ward shows that the failure rate can be reduced to nil by quick analysis of a second drop. The failure rate for RLpH and RLL analysis in our study was $23 \%$ and $43 \%$ respectively, which is in line with other studies: $10-20 \%$ for $\mathrm{pH}$ only and $>50 \%$ for simultaneous $\mathrm{pH} /$ lactate analysis (2). The required sample volume and the way the blood gas analyzer handles the sample can explain this large difference in failure rates between SSL and RLL/RLpH. Standard blood gas analyzers, including the RapidLab 860, require a large volume of 50-100 $\mu \mathrm{L}$ for determining $\mathrm{pH}$ and lactate results. If the sampling volume is too small, at best only $\mathrm{pH}$ is measured. SSL has the convenience of a very low sampling volume $(0.7 \mu \mathrm{L})$. This indicates that SSL is an attractive tool in the obstetric setting.

FBS with $\mathrm{pH}$ and/or lactate analysis can be used to discriminate the fetus that tolerates labor well from the fetus with a developing acidemia in case of a non-reassuring CTG. As presented in other studies, we found that the correlation between SSL and $\mathrm{pH}$ in fetal scalp blood is poor and that discrepancies are quite common (14). As both parameters might reflect different aspects of a developing acidemia, one may question whether discrepancies should be considered as false positive or false negative test results, or that $\mathrm{pH}$ is not the correct gold standard. A low $\mathrm{pH}$ with a normal lactate can be due to: 1) Transient impairment of the carbon dioxide exchange following umbilical cord compression. 2) Early stage of a fast-developing acidosis. 
A normal $\mathrm{pH}$ with a high lactate can be explained by: 1) Slow clearance of lactate from the fetal circulation and fast normalization of $\mathrm{pH}$ sometime after a hypoxic insult, 2) Early stage of a slowly developing acidosis, 3) Factors other than hypoxia, such as contamination of amniotic fluid or limited local perfusion as with caput formation $(8,12,15,16)$. Furthermore, the fetal acid-base balance is influenced by the administration of beta-mimetic drugs glucose, maternal hyperventilation and catecholamine concentrations (18). It therefore might be wise to perform parallel analyses of both $\mathrm{pH}$ and lactate in FBS, as they might mirror different stages of a developing asphyxia.

Concern has been raised that combined sampling ( $\mathrm{pH}$ and lactate) might increase the rate of operative delivery for fetal distress, due to an increased rate of abnormal results compared to single tests. Liljestrom et al. showed that combined sampling results in a $66 \%$ operative delivery rate versus $95 \%$ if single testing is used ( $\mathrm{pH}$ or lactate) $(7$, 14). One may argue that the use of a test with a $20 \%$ failure rate is not justified. We identified 5\% and 14\% discrepancies between SSL and RLpH for the cut-off values for reassuring fetal status and immediate intervention, respectively. Because of these discrepancies and the lack of a randomized controlled trial comparing combined testing with single testing, we propose combined sampling to reduce the chance of missing fetal hypoxia. In order to avoid repeated sampling when $\mathrm{pH}$ analysis fails, clinical management in those cases is based on SSL only. A summary of the clinical guideline we have implemented in our labor ward is provided in Table 3.

Table 3. Clinical guidelines for fetal scalp blood sampling using $\mathrm{pH}$ and Statstrip Lactate (SSL)

\begin{tabular}{lccc}
\hline & Normal & Borderline & Abnormal \\
\hline $\mathrm{pH}$ & $>7.25$ & $7.20-7.25$ & $<7.20$ \\
$\mathrm{SSL}(\mathrm{mmol} / \mathrm{l})$ & $<5.7$ & $5.7-7.0$ & $>7.0$ \\
\hline
\end{tabular}

- If $\mathrm{pH}$ fails, clinical management is based on SSL result only

- In the borderline group, repeated sampling in 20-30 minutes is recommended

- In case of discrepancies ( $\mathrm{pH}$ normal, SSL borderline/abnormal or vice versa); seek senior consultancy >> repeated sampling or delivery

The goal of FBS is to prevent adverse neonatal outcome. The commonly accepted thresholds for scalp $\mathrm{pH}$ are $\leq 7.20$ and $\geq 7.25$ for immediate delivery and reassuring fetal status. In a population of 486 fetuses in whom FBS was performed, we found that these values correspond to the $15^{\text {th }}$ and $25^{\text {th }}$ percentile respectively (12). In 
analogy with this, the $75^{\text {th }}$ and $85^{\text {th }}$ percentile cut-off values for RLL are $\leq 5.4$ and $\geq 6.6 \mathrm{mmol} / \mathrm{L}$ (12). The cut-off values for $\mathrm{SSL} \leq 5.7$ and $\geq 7.0 \mathrm{mmol} / \mathrm{L}$ were established by regression analysis of RL and SSL in 38 FBS samples from a previous study of our group (10). The cut-off for SSL of $7.0 \mathrm{mmol} / \mathrm{l}$ is close to the cutoff established by Ridenour et al. They compared Lactate Pro and Lactate Plus (an earlier version of SSL) with a lactate reference method using neonatal cord blood. Based on both regression and ROC curve analysis they found cut-offs to predict scalp $\mathrm{pH}<7.20$ at 7.0 and $6.8 \mathrm{mmol} / \mathrm{l}$, respectively. They concluded that sensitivity and specificity of Lactate Pro and Lactate Plus for predicting acidosis are nearly identical, suggesting that there is no difference in the ability of the devices to predict acidosis but that different cut-offs for this purpose must be used. As expected and in line with Ridenour et al., there was poor agreement between SSL and RLpH (20). We found strong agreement and a low discrepancy rate between the POC device (SSL) and the reference method (RLL). To our knowledge this comparison has never been made in a clinical setting. Although the cut-off values for SSL need to be further tested prospectively in clinical outcome studies, the above suggests that thresholds defined for SSL seem to be adequate.

Several limitations can be identified in our study. Firstly, in 76 samples SSL analysis was not performed at all. This was probably due to the fact that our labor ward staff was not yet familiar with the device. Secondly, we were not able to relate the threshold values to neonatal outcome, as the time interval between FBS and delivery varied from 10 minutes to 8 hours. Our study design did not allow for such analysis as we did not use the SSL values for clinical management. This is the scope for future research. Thirdly, our population contained only a few samples representing fetal acidosis. However, the low prevalence of poor outcome is a common problem in evaluating tests for fetal well-being during labor. FBS is an important tool to exclude acidosis when the CTG is abnormal, as the intra partum CTG has a high false positive rate for this outcome.

In conclusion, we showed that SSL is a reliable test and can be used in daily obstetric practice for POC lactate testing in fetal scalp blood. SSL provides accurate information on fetal status in the vast majority of cases at a minimal failure rate. As there are discrepancies between SSL and RLpH and the cut-off values have not yet been evaluated prospectively regarding intervention rates and neonatal outcome, we propose combined testing of SSL and $\mathrm{pH}$ in FBS. 


\section{References}

(1) Tuffnell D, Haw WL, Wilkinson K. How long does a fetal scalp blood sample take? Br J Obstet Gynaecol. 2006;113(3): 332-4.

(2) Westgren M, Kruger K, Ek S, Grunevald C, Kublickas M, Naka K, et al. Lactate compared with $\mathrm{pH}$ analysis at fetal scalp blood sampling: a prospective randomised study. Br J Obstet Gynaecol. 1998;105(1):29-33.

(3) Low JA, Lindsay BG, Derrick EJ. Threshold of metabolic acidosis associated with newborn complications. Am J Obstet Gynecol. 1997;177(6):1391-4.

(4) Low JA, Panagiotopoulos C, Derrick EJ. Newborn complications after intrapartum asphyxia with metabolic acidosis in the term fetus. Am J Obstet Gynecol. 1994;170(4):1081-7.

(5) Kruger K, Hallberg B, Blennow M, Kublickas M, Westgren M. Predictive value of fetal scalp blood lactate concentration and $\mathrm{pH}$ as markers of neurologic disability. Am J Obstet Gynecol. 1999;181(5 Pt 1):1072-8.

(6) Nordstrom L, Ingemarsson I, Kublickas M, Persson B, Shimojo N, Westgren M. Scalp blood lactate: a new test strip method for monitoring fetal wellbeing in labor. $\mathrm{Br} \mathrm{J}$ Obstet Gynaecol. 1995;102(11):894-9.

(7) Wiberg-Itzel E, Lipponer C, Norman M, Herbst A, Prebensen D, Hansson A, et al. Determination of $\mathrm{pH}$ or lactate in fetal scalp blood in management of intrapartum fetal distress: randomised controlled multicenter trial. BMJ. 2008;336(7656):1284-7.

(8) Allen RM, Bowling FG, Oats JJ. Determining the fetal scalp lactate level that indicates the need for intervention in labor. Aust N Z J Obstet Gynaecol. 2004;44(6):549-52.

(9) Nordstrom L. Fetal scalp and cord blood lactate. Best Pract Res Cl Ob. 2004;18(3):46776.

(10) Heinis AM, Dinnissen J, Spaanderman ME, Lotgering FK, Gunnewiek JM. Comparison of two point-of-care testing (POCT) devices for fetal lactate during labor. Clin Chem Lab Med. 2012;50(1):89-93.

(11) Ayres-de-Campos D, Bernardes J, Subcommittee F. Twenty-five years after the FIGO guidelines for the use of fetal monitoring: time for a simplified approach? Int J Gynecol Obstet. 2010;110(1):1-6.

(12) Heinis AM, Spaanderman ME, Gunnewiek JM, Lotgering FK. Scalp blood lactate for intra-partum assessment of fetal metabolic acidosis. Acta Obstet Gynecol Scand. 2011;90(10):1107-14.

(13) Bretscher J, Saling E. pH values in the human fetus during labor. Am J Obstet Gynecol. 1967;97(7):906-11.

(14) Liljestrom L, Wikstrom AK, Hanson U, Akerud H, Jonsson M. Evaluation of the discrepancy between $\mathrm{pH}$ and lactate in combined fetal scalp blood sampling. Acta Obstet Gynecol Scand. 2011;90(10):1088-93.

(15) Ingemarsson I, Arulkumaran S. Fetal acid-base balance in low-risk patients in labor. Am J Obstet Gynecol. 1986;155(1):66-9. 
(16) Engidawork E, Chen Y, Dell'Anna E, Goiny M, Lubec G, Ungerstedt U, et al. Effect of perinatal asphyxia on systemic and intracerebral $\mathrm{pH}$ and glycolysis metabolism in the rat. Exp Neurol. 1997;145(2 Pt 1):390-6.

(17) Schmidt S, Langner K, Dudenhausen JW, Saling E. Measurement of transcutaneous pCO2 and pO2 in the fetus during labor. Arch Gynecol Obstet. 1985;236(3):145-51.

(18) Nordstrom L, Ingemarsson I, Westgren M. Fetal monitoring with lactate. Baillieres Clin Obstet Gynaecol. 1996;10(2):225-42.

(19) Reif P, Lakovschek I, Tappauf C, Haas J, Lang U, Scholl W. Validation of a point-ofcare (POC) lactate testing device for fetal scalp blood sampling during labor: clinical considerations, practicalities and realities. Clin Chem Lab Med. 2014;52(6):825-33.

(20) Ridenour RV, Gada RP, Brost BC, Karon BS. Comparison and validation of point of care lactate meters as a replacement for fetal $\mathrm{pH}$ measurement. Clin Biochem. 2008;41(18):1461-5. 



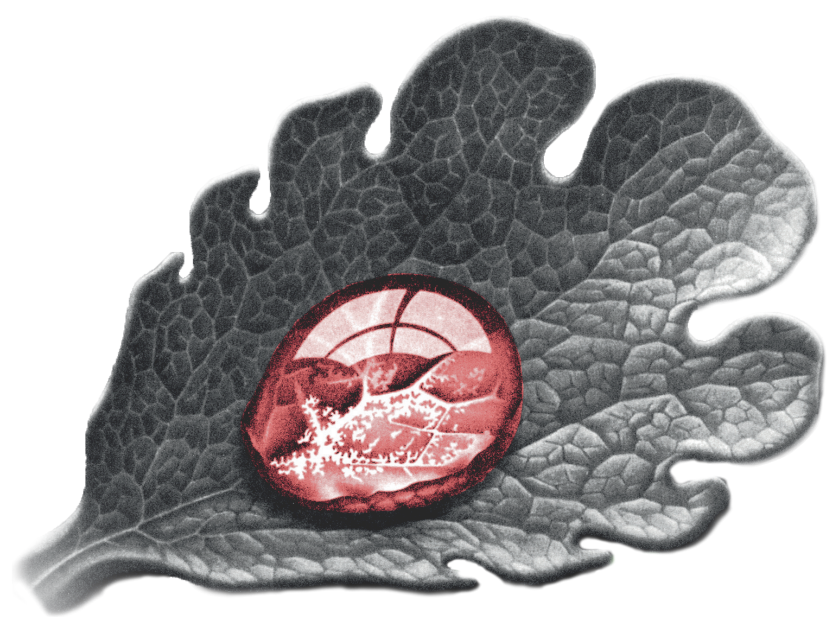




\section{Chapter 6}

TWO-YEAR FOLLOW UP OF AN EXPANDED FETAL MONITORING PROTOCOL AIMED AT INCREASING THE SUCCESS RATE OF FETAL BLOOD SAMPLING

Ayesha Heinis, Joris Van Drongelen, Frank Vandenbussche'Jeroen van Dillen 


\section{Abstract}

Introduction: To improve intra partum fetal monitoring in our hospital, we implemented two new procedures: ST-analysis of the fetal ECG and Point of Care (POC) lactate testing in fetal scalp blood sampling (FBS) in parallel with $\mathrm{pH}$ according to a new clinical guideline. In this study, we describe the two-year follow-up of these measures.

Materials and methods: We compared two birth cohorts with singleton vertex deliveries $\geq 36$ weeks in which FBS was performed. Cohort one ranged from 2010-2011, cohort two from 2014-2016. We assessed maternal and neonatal data, including mode of delivery, birthweight, Apgar score, FBS and cord blood acid-base values. Main outcome measures were FBS failure rate, operative delivery rates and metabolic acidosis at birth.

Results: Overall FBS usage remained stable with 137/1165 deliveries (12\%) in cohort 1 and 253/2139 (12\%) in cohort 2. The FBS failure rate decreased from $23 \%$ to $2 \%$, as did the number of samples per labor (from 2.3 to 1.6). In cases where FBS was performed, we found a significant decrease in ventouse delivery (from $37 \%$ to $27 \%$ ) and a significant increase in spontaneous vaginal delivery (from 37\% to 49\%). Low Apgar score and neonatal metabolic acidosis remained stable.

Conclusions: The implementation of STAN and POC lactate testing resulted in a tenfold decrease in FBS failure rate and fewer scalp samples per labor. This was associated with a higher proportion of spontaneous vaginal deliveries with comparable perinatal outcome. 


\section{Introduction}

Intra-partum cardiotocography (CTG) is a useful screening test for fetal hypoxia/ acidosis, but requires supplementary tests to avoid unnecessary operative delivery $(1,2)$. Many methods have been proposed for this purpose. The two best known and accepted ones are fetal blood sampling (FBS) and ST analysis of the fetal electrocardiogram (STAN).

The use of FBS in addition to CTG is likely to reduce the rate of cesarean sections (CS), when compared with CTG surveillance only $(3,4)$. Traditionally, $\mathrm{pH}$ is the primary parameter measured in FBS. This is cumbersome because a relatively large amount of blood (30-50 $\mu$ l) is needed. This leads to high sampling failure rates (up to $20 \%$ have been reported) and may lead to a subsequent delay in clinical management (5). Moreover, when only $\mathrm{pH}$ is used, no differentiation is made between respiratory (CO2 accumulation) and metabolic academia (lactate acidosis), the latter being associated with poor neonatal outcome (6). Lactate measurement in FBS may be an attractive alternative to $\mathrm{pH}$. Point of Care (POC) lactate testing devices need only a tiny amount of blood $(0.7-5 \mu \mathrm{l})$, deliver fast results (13-60 seconds) with a high success rate (97-99\%) (7). A randomized controlled trial (RCT), comparing $\mathrm{pH}$ and lactate, showed significantly fewer failures with lactate analysis (1 versus $20 \%$ ) and no differences in short term neonatal outcome or mode of delivery (8).

STAN is a technique that detects changes in the ST segment of the fetal ECG (ST events) during labor, which are an indicator of fetal hypoxia (9). The occurrence of these ST events is interpreted together with CTG, as defined in the STAN clinical guidelines (10). Meta-analysis comparing STAN to CTG with or without FBS, confirms a lower rate of metabolic acidosis (RR 0.64, 95\% CI 0.46-0.88), a reduction in the rate of FBS (RR 0.59, 95\% CI 0.45-0.79) and a reduction in the overall rate of assisted vaginal delivery (RR $0.92,95 \%$ CI 0.86-0.99). No reduction is shown in the rate of operative delivery for fetal distress, perinatal death, seizures and encephalopathy (11). Two studies have shown that in comparison to CTG with FBS, STAN is cost-effective $(12,13)$.

To improve intra partum fetal monitoring in our hospital, we expanded our procedures and techniques for fetal monitoring. STAN was installed in all delivery rooms by March 2012. At that time, FBS pH measurement as an adjunct to CTG was used at a frequency of around $11 \%$ of deliveries, but with a failure rate of around $20-30 \%$. In order to reduce this unacceptably high failure rate, we implemented POC lactate testing in conjunction with FBS $\mathrm{pH}$ measurement. 
In this paper, we describe the results of the two-year follow-up post implementation of these measures and the impact on FBS success, delivery intervention rates and perinatal outcome. 


\section{Materials and methods}

The study was conducted by comparing two cohorts with singleton vertex deliveries at the Radboud University Medical Center from $\geq 36$ weeks of gestation. Cohort I ranged from February $13^{\text {th }} 2010$ until March $13^{\text {th }}$ 2011. Cohort II ranged from February $5^{\text {th }} 2014$ until February $5^{\text {th }} 2016$.

The Radboud University Medical Center is a tertiary referral center with approximately 1500 deliveries per year (low, medium and high-risk). Deliveries are managed by clinical midwives and residents, supervised by obstetricians. Intra partum fetal monitoring is performed by CTG with the option to perform FBS in case of an intermediary or abnormal CTG trace, as interpreted by the obstetric caregiver.

The expanded fetal monitoring protocol was introduced from March 2012 and included the following:

1. STAN $^{\circ}$, Neoventa Medical, Gotenborg Sweden was introduced in all six delivery rooms. The classification of CTG tracings and ST-events is performed according to the STAN clinical guidelines (10). FBS in case of STAN use is recommended in the following three situations: (i) start of STAN ${ }^{\bullet}$ registration with an intermediary or abnormal CTG trace; (ii) abnormal CTG trace for more than 60 minutes during the first stage without ST events; and (iii) poor ECG signal quality in the presence of an intermediary or abnormal CTG trace (9).

2. FBS with POC lactate testing using Statstrip ${ }^{\bullet}$ Lactate (SSL) (Nova Biomedical, Waltham, MA, USA). SSL is a hand-held single-use device that utilizes $0.7 \mu \mathrm{L}$ of blood and electrochemical test strips. The result is available in 13 seconds.

FBS is carried out with the standard technique with the use of Rocket ${ }^{\bullet}$ disposable sampling kit (Rocket Medical, Washington USA). After incision of the fetal scalp, blood is collected in two heparinized plastic capillaries. In the first sample, POC lactate testing is performed with SSL. The second sample is sent to the laboratory for $\mathrm{pH}$ analysis on a blood gas analyzer (RapidLab 348, Siemens Healthcare Diagnostics, Deerfield, IL, USA). The result is available after 10-15 minutes. Because the SSL cutoff values were based on a small series of measurements, and not yet prospectively tested regarding intervention rates and neonatal outcome, we use combined testing of SSL and $\mathrm{pH}$ in the following manner: If $\mathrm{pH}$ fails, clinical management is based on the SSL result only. In case of discrepancies ( $\mathrm{pH}$ normal, SSL borderline/abnormal or vice versa) the team was instructed to consult a senior obstetrician to decide what 
to do: intra uterine resuscitation, repeated sampling or initiate immediate delivery. Cut-off values for SSL were: $<5.7 \mathrm{mmol} / \mathrm{L}$ reassuring; continue fetal monitoring, 5.7-7.0 mmol/L borderline; repeated sampling within 30 minutes and $>7.0 \mathrm{mmol} / \mathrm{L}$ abnormal; consider immediate delivery (14). For $\mathrm{pH}$, the corresponding cut-off values were: $>7.25$ (reassuring), 7.25 and 7.20 (borderline) and $<7.20$ (abnormal).

In order to keep the FBS rate at a reasonable level and the efficacy as high as possible, our local guideline regarding intra partum fetal monitoring was revised. The indication for FBS has become stricter and depends on the type of fetal monitoring used: CTG only or STAN. The new local guideline is based on the national one by the Dutch Society of Obstetricians and Gynecologists (NVOG) and provides an association between CTG classification and subsequent clinical management (15). For monitoring with CTG only: normal tracings - no actions required; suboptimal tracings - continue monitoring and try to alleviate possible causes (such as reducing maternal temperature, discontinuing oxytocin, changing maternal position, treating maternal hypotension etc.); abnormal tracings - try to alleviate possible causes. If reversal is not shown, perform FBS with immediate delivery in mind; pre-terminal tracing - immediate delivery. For monitoring with STAN: in order to prevent unnecessary CS when significant ST-events are present in the first stage of labor, FBS can be performed at the discretion of the obstetrician in those situations. The rationale behind this is that in the majority of FBS samples (56\%) taken in the presence of significant ST-events a $\mathrm{pH} \geq 7.25$ is found (16).

For both cohorts, maternal and neonatal data were derived from our local electronic patient file system. This included maternal age, parity, gestational age, mode of delivery, birth weight and Apgar score, as well as FBS and cord blood data. The success rate of FBS was defined as having a result with either method ( $\mathrm{pH}$ and/ or SSL). Metabolic acidosis at birth was systematically evaluated by cord blood sampling immediately after delivery from both the umbilical artery and vein. The umbilical vein specimen results were used to verify correct sampling. Only data from the umbilical artery specimens were used in the analysis under the assumption that these reflect fetal condition best. Cord blood samples were analyzed on the Rapid Point 500 (Siemens Healthcare Diagnostics, Deerfield, IL, USA). Base deficit was calculated for the blood compartment $[\mathrm{BD}(\mathrm{B})]$, using the algorithm: $\mathrm{BD}(\mathrm{B})=(1-$ $\left.0.014 \times c t \mathrm{Hb})\left[c \mathrm{HCO}_{3}^{-}-24.8\right)+(1.43 \times c t \mathrm{Hb}+7.7)(\mathrm{pH}-7.40)\right]$. Severe metabolic acidosis at birth was defined as $\mathrm{pH}<7.05$ and $\mathrm{BD}>12.0 \mathrm{mmol} / \mathrm{l}$ in cord artery blood (8). 
Statistical analysis was performed with SPSS version 22 (IBM SPSS Inc, Armonk, New York. Data are presented as numbers with percentages or medians with 95 (2.5-97.5) centile ranges. For normally distributed data, Student t-test, and for $2 \times 2$ tables, Chi square test was used. For skewed data, Mann-Withney $\mathrm{U}$ test was used. A $\mathrm{p}$ value $<0.05$ was considered as a statistically significant difference.

As confirmed by the Medical Ethics Committee of Radboud UMC, Nijmegen, the Netherlands, our study only involves existing clinical patient data and imposes no changes in general practice. Therefore, according to the Declaration of Helsinki, no ethical approval was required. 


\section{Results}

The total number of singleton vertex deliveries beyond 36 weeks in cohort one was 1165 and 2139 in cohort two. FBS was performed in 137 (12\%) deliveries in cohort one and $253(12 \%)$ in cohort two. The intra partum characteristics of the mother and baby pairs are summarized in Table 1 . The number of FBS samples per labor decreased from 2.3 in cohort one, to 1.6 in cohort two. Maternal characteristics and neonatal outcome were comparable. No complications of the FBS procedure were reported. The FBS success rate increased from $77 \%$ in FBS cohort one to $98 \%$ in FBS cohort two. There was a significant decrease in ventouse delivery resulting in a significant increase in spontaneous vaginal delivery.

Table 1. Intra partum characteristics of mother and baby pair cases where FBS was performed.

\begin{tabular}{|c|c|c|c|}
\hline Characteristic & $\begin{array}{c}\text { FBS Cohort } 1 \\
\mathbf{n}=137\end{array}$ & $\begin{array}{c}\text { FBS Cohort } 2 \\
\mathbf{n}=253\end{array}$ & P-value \\
\hline Maternal age (years) ${ }^{a}$ & $30(19-41)$ & $31(21-41)$ & 0.17 \\
\hline Nulliparous ${ }^{\text {b }}$ & $110(80)$ & $183(72)$ & 0.12 \\
\hline Gestational age (days) ${ }^{a}$ & $278(252-293)$ & $273(252-294)$ & 0.36 \\
\hline Number of FBS samples ${ }^{c}$ & $315(2.3)$ & $414(1.6)$ & $<0.0001$ \\
\hline Successful FBS b,d & $250(77)$ & $405(98)$ & $<0.00001$ \\
\hline Spontaneous vaginal delivery ${ }^{\mathrm{b}}$ & $50(37)$ & $123(49)$ & 0.026 \\
\hline Ventouse delivery & $52(37)$ & $69(27)$ & 0.04 \\
\hline Cesarean section & $35(25)$ & $60(24)$ & 0.72 \\
\hline Birth weight (grams) a & $3383(2360-4375)$ & $3245(2240-4329)$ & 0.05 \\
\hline Five minute Apgar score $<7^{b}$ & $2(1)$ & $11(4)$ & 0.10 \\
\hline Metabolic acidosis at birth ${ }^{\mathrm{b}, \mathrm{e}}$ & $4(3)^{*}$ & $9(4)^{\#}$ & 0.61 \\
\hline
\end{tabular}

Data are medians with $2.5^{\text {th }}$ and $97.5^{\text {th }}$ centile ${ }^{\mathrm{a}}$, numbers and percentage of total (\%) ${ }^{\mathrm{b}}$, or numbers and numbers per patient ${ }^{c}$. ${ }^{d}$ Successful analysis of total FBS samples ${ }^{\mathrm{e}} \mathrm{pH}<7.05$ and base deficit $>12 \mathrm{mmol} / \mathrm{l}$ in umbilical artery blood. "Time interval FBS-delivery: 26-580 minutes. ${ }^{*}$ Time interval FBS-delivery: 18-320 minutes

In cohort two, the total of FBS samples was 414 from 259 labors. The success rate of FBS-pH analysis was $83 \%$. From the $70(17 \%)$ cases where $\mathrm{pH}$ analysis failed, SSL analysis failed in six cases and was not performed in three cases. Of the remaining 61 cases where SSL data was available, $29(48 \%)$ women had a spontaneous vaginal 
delivery, $15(25 \%)$ a ventouse delivery and 16 (26\%) an emergency CS. From these 61 cases, one infant had metabolic acidosis at birth. The FBS sampling to delivery interval in this case was 189 minutes.

In 321 fetal scalp blood samples both SSL and $\mathrm{pH}$ results were available (Figure 1). In $91 \%(292 / 321)$ of the samples there was concordance between SSL and pH regarding the presence (SSL $>7.0 \mathrm{mmol} / \mathrm{l}$ and $\mathrm{pH}<7.20$ ) or absence of acidemia $(\mathrm{SSL}<5.7 \mathrm{mmol} / \mathrm{L}$ and $\mathrm{pH}>7.25)$. There was a discrepancy between SSL and $\mathrm{pH}$ regarding the presence (15 samples) and absence (14 samples) of acidemia in $9 \%$ (29/321) of the samples.

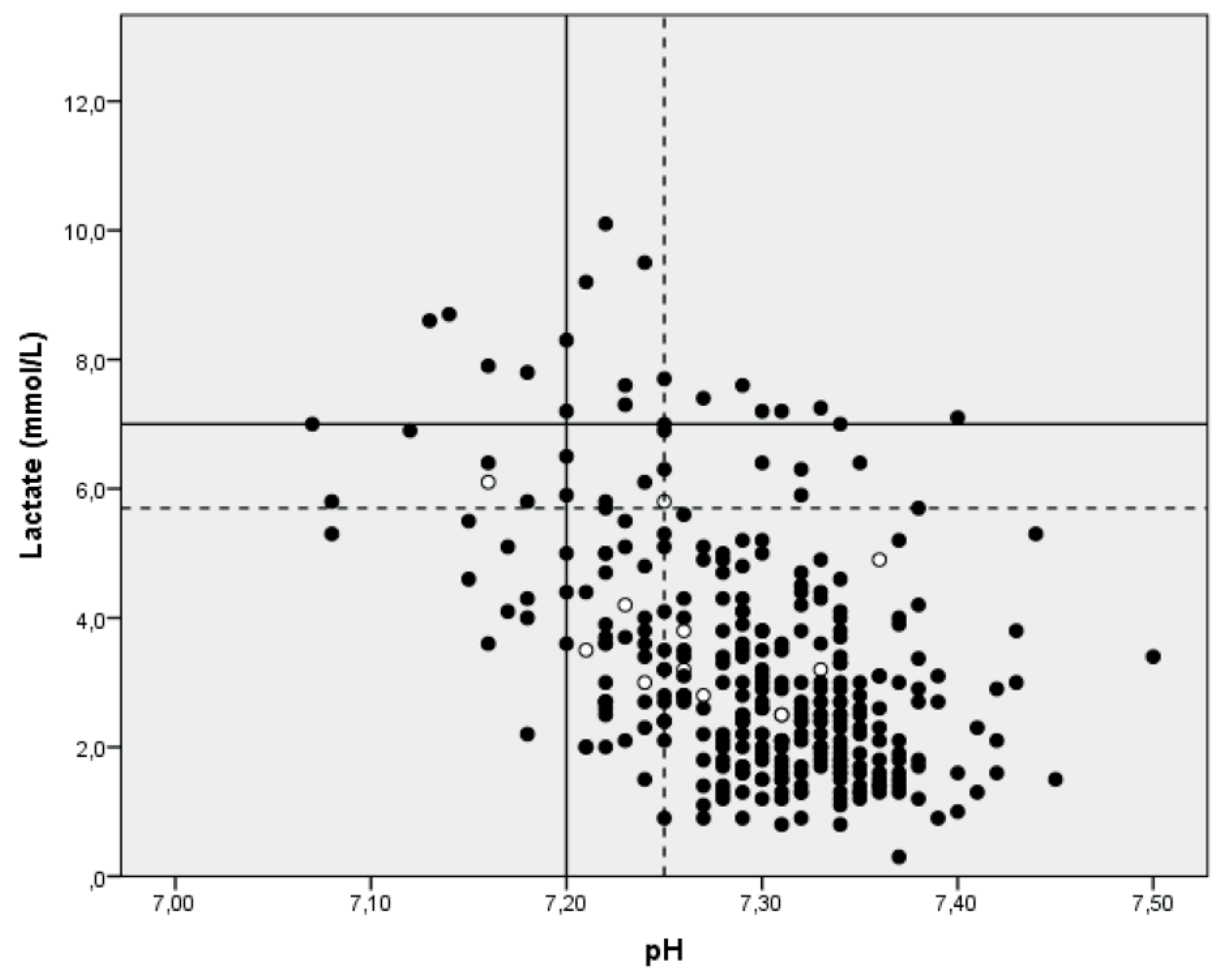

Figure 1. Scatterplot of 321 lactate determinations with Statstrip Lactate (SSL) and pH. Dashed lines correspond to cut-off values for reassurance (SSL $5.7 \mathrm{mmol} / \mathrm{L}$ and pH 7.25). Continuous lines correspond to cut-off values for immediate delivery (SSL $7.0 \mathrm{mmol} / \mathrm{L}$ and pH 7.20). Open dots are FBS samples from infants with metabolic acidosis at birth. 
Table 2 shows the nine individual cases of metabolic acidosis at birth (umbilical artery $\mathrm{pH}<7.05$ and $\mathrm{BD}>12 \mathrm{mmol} / \mathrm{l}$ ) from cohort 2 , tabulated in order of FBS sampling to delivery interval. Acute obstetric complications including placental abruption, uterine rupture, or prolapsed cord, were absent. In the five cases where the sampling to delivery interval was $<60$ minutes, $\mathrm{pH}$ was more often abnormal than SSL. In the four cases where the sampling to delivery interval exceeded 60 minutes, SSL and $\mathrm{pH}$ results were normal. Neonatal course was uneventful in all infants.

Table 2. Nine cases of metabolic acidosis at birth (umbilical artery $\mathrm{pH}<7.05$ and base deficit $>12 \mathrm{mmol} / \mathrm{l}$ ), tabulated in order of time interval between fetal blood sampling and delivery. In case of multiple FBS samples, the values of the last FBS sample are shown.

\begin{tabular}{lcccccccc}
\hline No & $\begin{array}{c}\text { Time } \\
\text { interval }\end{array}$ & AS 5 & $\begin{array}{c}\text { FBS } \\
\text { SSL }\end{array}$ & $\begin{array}{c}\text { FBS } \\
\mathbf{p H}\end{array}$ & $\begin{array}{c}\text { UA } \\
\text { Lactate }\end{array}$ & $\begin{array}{c}\text { UA } \\
\mathbf{p H}\end{array}$ & $\begin{array}{c}\text { UA } \\
\text { BD }\end{array}$ & $\begin{array}{c}\text { Mode of } \\
\text { delivery }\end{array}$ \\
\hline 1 & $0: 18$ & 9 & 6.1 & 7.16 & 9.9 & 7.02 & 15.0 & VE \\
2 & $0: 18$ & 8 & FA & 7.15 & 10.2 & 6.91 & 16.9 & VE \\
3 & $0: 33$ & 8 & 4.4 & 7.32 & 12.7 & 7.01 & 15.2 & VE \\
4 & $0: 35$ & 8 & 4.2 & 7.23 & NA & 6.98 & 12.8 & VE \\
5 & $0: 36$ & 9 & 3.5 & 7.21 & 14.2 & 6.81 & 21.3 & CS \\
6 & $1: 04$ & 10 & 2.7 & 7.22 & 8.0 & 7.03 & 13.9 & VE \\
7 & $1: 19$ & 8 & 4.9 & 7.28 & NA & 7.02 & 14.2 & VE \\
8 & $1: 30$ & 8 & 3.3 & 7.28 & NA & 7.02 & 13.7 & SV \\
9 & $1: 46$ & 7 & 2.6 & 7.30 & 10.8 & 6.86 & 14.4 & VE \\
\hline
\end{tabular}

AS; Apgar score, SSL; Statstrip Lactate, UA; umbilical artery, BD; base deficit, FA; failed analysis, NA; not available VE; vacuum extraction, SV; spontaneous vaginal delivery, CS; Cesarean section. 


\section{Discussion}

After implementation of the expanded fetal monitoring protocol we found an equal number of deliveries in which FBS was performed, a reduction in repeated FBS per labor, a much higher FBS success rate, and a higher proportion of spontaneous vaginal deliveries with comparable perinatal outcome.

FBS is used as an adjunct to CTG in order to identify the fetus at risk for hypoxia/ acidosis. Its use varies between countries, hospitals and obstetric care providers. In the Netherlands FBS is performed in 3 to $15 \%$ of deliveries (17). Although there was a decrease in the number of scalp samples per labor from 2.3 to 1.6 in our study, we did not see a reduction in overall FBS usage as reported in other studies $(9,11)$. An explanation for this could be that the residents, midwives and obstetricians on our ward were not completely familiar with the STAN technique and therefore did not dare to entirely rely on the STAN clinical guidelines. Moreover, our guideline allowed for FBS to be performed in case of significant ST events in the first stage of labor, as it has been reported that the majority (56\%) of FBS samples taken in those situations show a $\mathrm{pH} \geq 7.25$ (16). Secondly, the reduction in the number of FBS per woman suggests that the clinicians relied more on ST-analysis after a reassuring FBS-result. Therefore, the implementation of ST-analysis could explain both the absence of a reduction in overall FBS usage as well as the reduction in the number of repeated FBS per labor.

FBS as an adjunct to STAN is somewhat controversial and some argue that FBS should be abandoned entirely (18). However, false negative results have been reported with STAN use (19-21). In fact, the STAN clinical guidelines were modified due to the reports of these false negative results to include recommendations for situations in which additional fetal information is needed (10). The STAN clinical guidelines do not explicitly mention FBS as a possible means to provide this additional information, the national guideline on fetal monitoring issued by the Dutch Society of Obstetricians and Gynecologists (NVOG) does recommend FBS in those situations (15). Our updated local guideline is based on this national guideline and recommends a stricter use of FBS to make it as efficient as possible. FBS is an invasive test with rare but potentially serious complications including scalp abscess, bleeding and infection. These risks are comparable to the risk with the use of a scalp electrode (22). Moreover, the positive predictive value is low as the a priori risk of an acidotic fetus is low. The absence of a reduction in overall FBS usage found in our study, underlines the need for more training and critical discussion between colleagues. We also think that there should be a greater involvement of the supervising obstetrician 
prior to the decision to perform FBS, as this has shown to increase the pretest probability of detecting fetal acidosis and reduce the operative delivery rate for fetal distress, as well unnecessary FBS (23). Moreover, a large prospective observational study from Norway evaluating the clinical use of STAN, showed a low proportion of metabolic acidosis $(0.8 \%)$ with a restricted use of FBS (2.4\%) (24).

By implementing SSL for POC lactate testing in FBS, we have reduced the FBS-failure rate to $3.5 \%$. Recent experience with SSL on our ward shows that the failure rate can be reduced to nil by quick analysis of a second blood drop. This is in accordance with the literature where failure rates of $1-3 \%$ have been reported $(8,25,26)$. Prior to the implementation of SSL, the FBS-failure rate in our institution, was at an unacceptably high level of $20-30 \%$, which is much higher than the $10-20 \%$ reported in the literature (26-28). This high failure rate may have resulted in large sampling to result intervals and therefore in a delay in clinical management as Tufnell and coworkers previously reported (5). We decided to perform lactate testing before $\mathrm{pH}$ analysis, as SSL delivers a result in 13 seconds. If the value is abnormal, arrangements for delivery can be made while waiting for the $\mathrm{pH}$ result, thereby keeping a potential delay in management as low as possible. In our opinion, the CTG abnormalities indicating FBS should be severe enough to warrant immediate delivery. This means that if there is no result, immediate delivery should be performed. This could mean that a high FBS failure rate might also result in unnecessary operative intervention. With the use of SSL, we obtained a FBS result in 60/69 cases where $\mathrm{pH}$ analysis failed. Assuming that in those cases operative delivery would have been performed because of no $\mathrm{pH}$ result, one might argue that the availability of an SSL has prevented operative delivery in $44 / 60$ cases.

Concern has been raised that combined sampling ( $\mathrm{pH}$ and lactate) might increase the rate of operative delivery for fetal distress. Wiberg et al. reported in their RCT comparing $\mathrm{pH}$ with lactate in FBS a significantly higher proportion of abnormal results in the lactate group. In fact, this was the only statically significant difference in the trial. One might therefore argue that combined testing of $\mathrm{pH}$ and lactate would be a more reliable risk indicator of metabolic acidosis. The authors however, discouraged this as it might lead to an increased rate of abnormal results, and thereby the rate of operative interventions (8). Liljestrom et al, showed that combined testing resulted in a $66 \%$ operative delivery rate versus a $95 \%$ rate if single testing was used (29). This is in accordance with our study, where we showed that combined testing of lactate and $\mathrm{pH}$ results in a $12 \%$ increase in spontaneous vaginal deliveries, a $10 \%$ reduction in ventouse deliveries with a comparable cesarean section rate. 
FBS with $\mathrm{pH}$ and/or lactate analysis can be used to discriminate the fetus that tolerates labor well from the fetus with a developing acidemia in case of a non-reassuring CTG. The relationship between lactate and $\mathrm{pH}$ in fetal scalp blood is known to be poor and discrepancies are quite common (14, 27, 29, 30). As both parameters might reflect different aspects of a developing acidemia, one may question whether discrepancies should be considered as false positive or false negative test results, or that $\mathrm{pH}$ is not the correct gold standard. We thought that it might therefore be wise to perform parallel analyses of both $\mathrm{pH}$ and lactate in FBS, as they might mirror different stages of a developing asphyxia. In a previous study from which FBS cohort one originated, we identified 5\% and 14\% discrepancies between SSL and pH for the cut-off values for reassurance $(5.7 \mathrm{mmol} / \mathrm{L}$ and 7.25$)$ and immediate delivery (7.0 mmol/L and 7,20) (14). Assuming $\mathrm{pH}$ with cut-offs of 7.20 and 7.25 to be the gold standard, a 14\% discrepancy rate for immediate intervention could mean that cases of imminent hypoxia might be missed ("false negatives"). On the other hand, a large discrepancy rate for reassurance might increase intervention rates ("false positives"). In the present study, the discrepancy rates between SSL and $\mathrm{pH}$ for these cut-off values were equally distributed at $5 \%$ and $4 \%$ respectively. In addition to the absence of randomized controlled trials comparing combined with single testing, the use of SSL and pH in parallel with the use of these cut-off values seems to be justified.

Several limitations can be identified in our study. Firstly, this is a single center retrospective cohort study. Although data was collected and checked from multiple data sources from our laboratory and obstetric department, inaccurate record keeping or misclassification could have influenced outcome data. Secondly, we did not monitor the type of fetal monitoring used (e.g STAN or CTG only) and the indication for FBS, therefore we could not determine what caused the increase in FBS usage specifically. Thirdly, both FBS cohorts contained only a few samples representing fetal acidosis. However, the low prevalence of poor outcome is a common problem in evaluating tests for fetal well-being during labor. FBS is an important tool to exclude acidosis when the CTG is abnormal or, in case of STAN monitoring, if the physician is uncertain of fetal well-being and additional information is required.

In conclusion, we showed that the implementation of an expanded fetal monitoring protocol incorporating STAN and FBS lactate testing in conjunction with $\mathrm{pH}$, resulted in an unchanged overall FBS usage, a tenfold decrease in FBS failure rate and a lower rate of FBS per labor. This was associated with a higher proportion of 
spontaneous vaginal deliveries with comparable perinatal outcome. A continuous focus on the quality of intrapartum surveillance, by ongoing discussion and training is needed to reduce FBS usage and improve maternal and neonatal outcome. 


\section{References}

(1) Lotgering FK, Wallenburg HC, Schouten HJ. Interobserver and intraobserver variation in the assessment of antepartum cardiotocograms. American journal of obstetrics and gynecology. 1982;144(6):701-5.

(2) Rhose S, Heinis AM, Vandenbussche F, van Drongelen J, van Dillen J. Inter- and intraobserver agreement of non-reassuring cardiotocography analysis and subsequent clinical management. Acta Obstet Gynecol Scand. 2014;93(6):596-602.

(3) Haverkamp AD, Orleans M, Langendoerfer S, McFee J, Murphy J, Thompson HE. A controlled trial of the differential effects of intrapartum fetal monitoring. American journal of obstetrics and gynecology. 1979;134(4):399-412.

(4) Jorgensen JS, Weber T. Fetal scalp blood sampling in labor--a review. Acta Obstet Gynecol Scand. 2014;93(6):548-55.

(5) Tuffnell D, Haw WL, Wilkinson K. How long does a fetal scalp blood sample take? BJOG. 2006;113(3):332-4.

(6) Low JA, Panagiotopoulos C, Derrick EJ. Newborn complications after intrapartum asphyxia with metabolic acidosis in the term fetus. American journal of obstetrics and gynecology. 1994;170(4):1081-7.

(7) Heinis AM, Dinnissen J, Spaanderman ME, Lotgering FK, Gunnewiek JM. Comparison of two point-of-care testing (POCT) devices for fetal lactate during labor. Clin Chem Lab Med. 2011;50(1):89-93.

(8) Wiberg-Itzel E, Lipponer C, Norman M, Herbst A, Prebensen D, Hansson A, et al. Determination of $\mathrm{pH}$ or lactate in fetal scalp blood in management of intrapartum fetal distress: randomised controlled multicentre trial. BMJ. 2008;336(7656):1284-7.

(9) Westerhuis ME, Moons KG, van Beek E, Bijvoet SM, Drogtrop AP, van Geijn HP, et al. A randomised clinical trial on cardiotocography plus fetal blood sampling versus cardiotocography plus ST-analysis of the fetal electrocardiogram (STAN) for intrapartum monitoring. BMC Pregnancy Childbirth. 2007;7:13.

(10) Amer-Wahlin I, Arulkumaran S, Hagberg H, Marsal K, Visser GH. Fetal electrocardiogram: ST waveform analysis in intrapartum surveillance. BJOG. 2007;114(10):1191-3.

(11) Blix E, Brurberg KG, Reierth E, Reinar LM, Oian P. ST waveform analysis versus cardiotocography alone for intrapartum fetal monitoring: a systematic review and metaanalysis of randomized trials. Acta Obstet Gynecol Scand. 2016;95(1):16-27.

(12) Heintz E, Brodtkorb TH, Nelson N, Levin LA. The long-term cost-effectiveness of fetal monitoring during labor: a comparison of cardiotocography complemented with ST analysis versus cardiotocography alone. BJOG. 2008;115(13):1676-87.

(13) Vijgen SM, Westerhuis ME, Opmeer BC, Visser GH, Moons KG, Porath MM, et al. Cost-effectiveness of cardiotocography plus ST analysis of the fetal electrocardiogram compared with cardiotocography only. Acta Obstet Gynecol Scand. 2011;90(7):772-8. 
(14) Heinis A, van Dillen J, Oosting J, Rhose S, Vandenbussche F, Van Drongelen J. Clinical evaluation of Statstrip(R) Lactate for use in fetal scalp blood sampling. Acta Obstet Gynecol Scand. 2017;96(3):334-41.

(15) Nederlandse Vereniging voor Obstetrie en Gynaecologie. Richtlijn Foetale Bewaking [Fetal monitoring during labor]. Available from: www.nvog.nl; May 2016 [Dutch].

(16) Becker JH, Westerhuis ME, Sterrenburg K, van den Akker ES, van Beek E, Bolte AC, et al. Fetal blood sampling in addition to intrapartum ST-analysis of the fetal electrocardiogram: evaluation of the recommendations in the Dutch STAN(R) trial. BJOG. 2011;118(10):1239-46.

(17) Westerhuis ME, Strasser SM, Moons KG, Mol BW, Visser GH, Kwee A. [Intrapartum foetal monitoring: from stethoscope to ST analysis of the ECG]. Ned Tijdschr Geneeskd. 2009;153:B259.

(18) Chandraharan E. Fetal scalp blood sampling during labor: is it a useful diagnostic test or a historical test that no longer has a place in modern clinical obstetrics? BJOG. 2014;121(9):1056-60; discussion 60-2.

(19) Doria V, Papageorghiou AT, Gustafsson A, Ugwumadu A, Farrer K, Arulkumaran S. Review of the first 1502 cases of ECG-ST waveform analysis during labor in a teaching hospital. BJOG. 2007;114(10):1202-7.

(20) Ingemarsson I, Westgren M. ST analysis. BJOG. 2007;114(11):1445.

(21) Westerhuis ME, Kwee A, van Ginkel AA, Drogtrop AP, Gyselaers WJ, Visser GH. Limitations of ST analysis in clinical practice: three cases of intrapartum metabolic acidosis. BJOG. 2007;114(10):1194-201.

(22) Schaap TP, Moormann KA, Becker JH, Westerhuis ME, Evers A, Brouwers HA, et al. Cerebrospinal fluid leakage, an uncommon complication of fetal blood sampling: a case report and review of the literature. Obstet Gynecol Surv. 2011;66(1):42-6.

(23) Lowe B, Beckmann M. Involving the consultant before fetal blood sampling. Aust N Z J Obstet Gynaecol. 2016;56(4):387-90.

(24) Kessler J, Moster D, Albrechtsen S. Intrapartum monitoring of high-risk deliveries with ST analysis of the fetal electrocardiogram: an observational study of 6010 deliveries. Acta Obstet Gynecol Scand. 2013;92(1):75-84.

(25) Ramanah R, Martin A, Clement MC, Maillet R, Riethmuller D. Fetal scalp lactate microsampling for non-reassuring fetal status during labor: a prospective observational study. Fetal Diagn Ther. 2010;27(1):14-9.

(26) Westgren M, Kruger K, Ek S, Grunevald C, Kublickas M, Naka K, et al. Lactate compared with $\mathrm{pH}$ analysis at fetal scalp blood sampling: a prospective randomised study. Br J Obstet Gynaecol. 1998;105(1):29-33.

(27) Nordstrom L. Fetal scalp and cord blood lactate. Best Pract Res Clin Obstet Gynaecol. 2004;18(3):467-76.

(28) Nordstrom L, Persson B, Shimojo N, Westgren M. Fetal scalp and umbilical artery blood lactate measured with a new test strip method. Br J Obstet Gynaecol. 1992;99(4):3079 . 
(29) Liljestrom L, Wikstrom AK, Skalkidou A, Akerud H, Jonsson M. Experience of fetal scalp blood sampling during labor. Acta Obstet Gynecol Scand. 2014;93(1):113-7.

(30) Heinis AM, Spaanderman ME, Gunnewiek JM, Lotgering FK. Scalp blood lactate for intra-partum assessment of fetal metabolic acidosis. Acta Obstet Gynecol Scand. 2011;90(10):1107-14. 


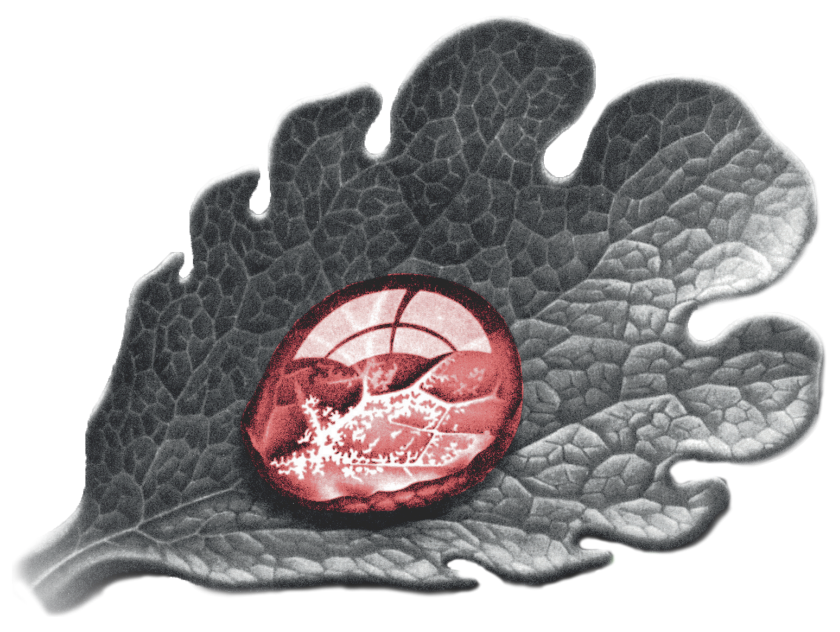




\section{Chapter 7}

GENERAL DISCUSSION AND CONCLUSIONS 



\section{General discussion}

The ideal method for intra partum fetal monitoring would be simple, noninvasive, and applicable for all women in labor, irrespective of gestational age. Furthermore, the method should ideally satisfy three criteria. Firstly, the method should give an early warning (when the baby is only pre-acidemic), to provide time for the clinician to deliver the baby without putting the mother at unnecessary risk. Secondly, system feedback should be clear ("do we have a problem? yes or no") and easy to follow guidelines should be available. Thirdly, the test should be able to identify the nonhypoxic fetuses so unnecessary intervention can be avoided. Neither FBS, nor STAN fulfill all of these criteria, but their integrated use could be the way to move forward.

In this thesis, we have elaborated further on these challenges, aimed at guiding best practice. In this chapter, a discussion of the main findings of this thesis is given, as well as the implications for clinical practice and future research. The main findings of this thesis are:

- The inter observer agreement on CTG patterns prior to FBS is poor. There is a relatively high chance that clinicians would disagree on the need to perform FBS. To ensure that FBS is as efficient as possible, the indication should be more strictly described in a well implemented guideline providing a clear association between the different CTG classifications and subsequent management (Chapter 2).

- The lactate concentration in fetal scalp blood correlates with both $\mathrm{pH}$ and base deficit. The test characteristics providing either reassurance or an indication for immediate delivery seem to be better for lactate than for $\mathrm{pH}$, base deficit, or the latter two combined. As lactate analysis is easier, faster, and more often successful, fetal scalp blood lactate is an attractive alternative to full blood gas analysis in FBS (Chapter 3).

- Laboratory evaluation shows that StatStrip Lactate can be used as a Point of Care (POC) device for lactate measurements in FBS. In comparison to Lactate Pro, StatStrip Lactate shows the better analytical performance characteristics, with the lowest coefficients of variance, the closest correlation to and the highest agreement with our reference method (Chapter 4). 
- Clinical evaluation shows that Statstrip Lactate accurately measures the lactate level in FBS with a minimal failure rate. Using the experimentally established cut-off values, Statstrip Lactate provides accurate information on fetal status in the vast majority of cases. The correlation between lactate and $\mathrm{pH}$ is poor and discrepancies are common. As both parameters might reflect different aspects of a developing acidemia and the cut-off values have not yet been evaluated and prospectively tested regarding intervention rates and neonatal outcome, we propose combined testing of Statstrip lactate and pH in FBS (Chapter 3 and 5).

- The implementation of an expanded fetal monitoring protocol containing combined testing of Statstrip lactate and pH, ST-analysis of the fetal ECG and an updated clinical guideline, results in an increase in FBS usage, a tenfold decrease in FBS failure rate, fewer scalp samples per labor and a higher proportion of spontaneous vaginal deliveries with comparable perinatal outcome (Chapter 6).

- A continuous focus on the quality of intrapartum surveillance, by critical discussion, training and involving the supervising obstetrician prior to the decision to perform FBS is needed to reduce FBS usage and improve maternal and neonatal outcome. (Chapter 2 and 6).

\section{$C T G$ and $F B S$}

FBS is performed as an additional test in case of CTG suspected fetal distress. As the interpretation of a non-reassuring or abnormal tracing CTG suffers from high inter-and intra-observer variation, the indication for FBS also carries a high risk of observer disagreement. In Chapter 2, we describe to what extent clinicians agreed on clinical management options when CTG abnormalities occurred.

Considerable variation in the classification of CTG patterns was found. Observers agreed poorly with each other and fair to good with themselves on CTG classification and clinical management. They achieved the highest agreement on abnormal CTG patterns and on the clinical management option "continue monitoring." Clinical midwives and registrars agreed better than the obstetricians. One may speculate on possible explanations: (i) registrars are all trained in the same teaching hospital, whereas obstetricians may have different backgrounds; (ii) clinical midwives and registrars are more "bed-side" than obstetricians and have more current experience than obstetricians; (iii) the observer category size differed in the various groups (four obstetricians, three registrars and two clinical midwives), increasing the probability that the fair to good agreement of the midwives was due to chance. It is likely that the 
level of experience and type of profession influences CTG interpretation. Westerhuis et al. showed differences indicative of higher rates of agreement in more experienced and recently educated professionals (1).

The observers assessed the CTG patterns using the FIGO/STAN guidelines, while the CTG patterns stem from a period of time in which the FIGO/STAN guidelines were not used in the labor ward. Probably FBS was performed on more liberal indications at that time (i.e. for reassurance on fetal condition before active pushing is started). Even with the use of the FIGO/STAN guidelines, the interpretation, i.e. classification of CTG patterns is difficult. This has clinical implications regarding the use of FBS and fetal monitoring with CTG. FBS is an invasive test and the a priori chance for a fetus suffering from acidosis is low. To ensure that FBS is as efficient as possible, we think the indication for its use should be more strictly described. Moreover, physicians should be aware of the fact that there is a relatively high chance their colleagues might classify a CTG pattern differently or choose another clinical management strategy. The success of FBS depends on correct CTG-interpretation and appropriate intervention (or non-intervention), which highlights the importance of critical discussion between colleagues and the need for a well implemented guideline providing a clear association between the different CTG classifications and subsequent management options.

Critics of FBS argue that it is an invasive test with potentially serious complications and that its predictive value for poor perinatal outcome is very low $(2,3)$. Both aspects need to be seen in perspective. Firstly, despite the large numbers of FBS performed worldwide over the last four decades, only a few serious complications have been reported. Reviewing the literature twelve papers reported a total of 37 cases of severe complications following FBS (4). Recent data from a large maternity unit in Denmark where FBS was used in over 25000 patients during a ten-year period, no such severe complications were observed (5). Secondly, the majority of babies depressed at birth, requiring intubation and ventilation, are not significantly hypoxic or acidemic. Instead their poor condition has other causes, such as sepsis, meconium aspiration and trauma. It would be unreasonable to expect such outcomes to be predictable by measuring the acid-base status of the fetus. Moreover, the prevalence of metabolic acidosis severe enough to cause brain damage is rare: 3/1000 (6). Therefore, no matter how excellent the test will be, the positive predictive value will still be low. On the flip side, the negative predictive value will be very high. Therefore, the main value of FBS is not to detect acidosis, but to exclude it when the CTG is abnormal. FBS samples from 486 fetuses born in our obstetric unit shows that the vast majority of $\mathrm{pH}$ values within the normal range (Chapter 3 ). This indicates that 
despite CTG abnormalities, most of the time a relatively normal $\mathrm{pH}$ and lactate is found in FBS. Therefore, if the only concern in labor is fetal hypoxia and the labor is otherwise progressing well, a normal $\mathrm{pH}$ or lactate from a free flowing FBS makes significant metabolic acidosis extremely unlikely, thus allowing additional time to achieve a vaginal delivery rather than requiring an urgent CS.

FBS has the potential to reduce the caesarean section rate. To show this, CTG with FBS needs to be compared to CTG alone. There is only one RCT that has made this direct comparison; the Denver trial of 1979. This was a three-armed RCT of 695 high-risk pregnancies. 232 were monitored by auscultation alone, 233 by CTG alone, and 230 by CTG and FBS. There was a $7 \%$ difference in CS rate in favor of CTG with FBS (7). This difference was not statistically significant. Since the Denver trial a number of other trials were performed to test the efficacy of CTG. These 11 trials are consolidated in a recent meta-analysis from 2013 (8). The conclusion was that continuous CTG during labor is associated with a reduction in neonatal seizures, but no significant differences in cerebral palsy, infant mortality or other standard measures of neonatal well-being. However, continuous CTG was associated with an increase in caesarean sections and instrumental vaginal births. Critics of FBS refer to this meta-analysis when they argue that there is no shown benefit of FBS as an adjunct to CTG. However, the comparison was made between RCT's with access to FBS in all arms and RCT's without FBS. I.e. intermittent auscultation with or without FBS was compared to CTG with or without FBS. If we look at the same data from the older version of this meta-analysis, the data were presented in a different way. Intermittent auscultation was compared to CTG alone and to CTG with FBS. Thus, allowing an indirect comparison between CTG with FBS and CTG without FBS. As compared to intermittent auscultation, the CS rate is almost doubled, whereas in the studies where FBS was used in addition to CTG, this increase was much less profound (9). Although high level evidence is lacking, this implies that FBS might reduce the CS rate with 6 to $7 \%$.

\section{Lactate, $p H$ or both?}

When Saling first introduced the technique of FBS, only $\mathrm{pH}$ was measured (10). The measurement of $\mathrm{pH}$ alone provides little information about the duration and extent of hypoxia/metabolic acidemia. Transient respiratory acidemia with high $\mathrm{pCO} 2$ is a common feature of normal labor and correlates poorly with neonatal outcome (6, 11). In contrast, severe metabolic acidosis represents tissue hypoxia and correlates well with neonatal multi-organ dysfunction and impaired neurodevelopment (12, 13). 
Base deficit (BD) is used to quantify metabolic acidosis, as it accounts for both the carbon and the non-carbon buffering systems (14). Full blood gas analysis requires a relatively large amount of blood, which is difficult to obtain by FBS, therefore resulting in rather high failure rates (15). Apart from this practical drawback, for various reasons $\mathrm{BD}$ values may not be the best representative of fetal metabolic status. $\mathrm{BD}$ is not measured but is calculated using $\mathrm{pH}$ and $\mathrm{pCO} 2$ values. As $\mathrm{CO} 2$ can cross the placenta rapidly when perfusion is restored, $\mathrm{pCO} 2$ and therefore $\mathrm{BD}$ can fluctuate. In addition, air contamination in FBS may produce incorrect $\mathrm{pCO} 2$ levels and consequently false $\mathrm{BD}$ values. Moreover, the algorithms used for $\mathrm{BD}$ calculation are attuned to adult hemoglobin type, oxygen saturation, and temperature, and not to fetal blood composition, thus causing a systematic error in BD calculation (16).

Lactate is the major end product of anaerobic metabolism and can be measured in a tiny amount of blood, which is practical in FBS. It has been well established by animal and human studies that fetal scalp blood lactate levels adequately represent lactate levels in the fetal central circulation and that fetal lactate production is endogenous in origin $(17,18)$. In recent years, lactate has been shown to be a good predictor of severe morbidity in neonates and in intra-partum surveillance $(19,20)$. As described in Chapter 3, the lactate concentration in FBS gradually increases with progressing metabolic acidosis and was a better marker than $\mathrm{pH}$ and/or $\mathrm{BD}$, both for reassurance and for the need for immediate intervention. As lactate analysis is easier, faster, and more often successful than full blood gas analysis, fetal scalp blood lactate is an attractive alternative to full blood gas analysis during labor.

As described in Chapter 3, a lactate value $<5.4 \mathrm{mmol} / \mathrm{l}$ indicated the $75 \%$ of reassuring cases, lactate between 5.4 and $6.6 \mathrm{mmol} / \mathrm{l}$ the $10 \%$ with inconclusive fetal status, and lactate $\geq 6.6 \mathrm{mmol} / \mathrm{l}$ the $15 \%$ of fetuses in need of immediate intervention because of marked metabolic acidosis. These lactate cut-off values should not be taken to represent universal absolute threshold values for at least two reasons. Firstly, the cut-off values were estimated on the basis of a retrospective cohort and need confirmation in an independent prospective cohort. Secondly, in the absence of international standards and reference material for calibration alignment between laboratory analyzers and point of care (POC) devices, our absolute values are valid only with the Rapidlab-860, which is a large blood gas analyzer requiring a minimum sampling volume of 50 microliters.

POC lactate testing devices are attractive for their use in FBS because of the low sampling volume $(0.7-5.0 / \mathrm{L})$, rapid results $(13-60 \mathrm{~s})$ and low failure rates $(1.3 \%-$ $2.9 \%)(15)$. However, due to lack of standardization, test characteristics and lactate 
cut-off values in FBS have to be determined for each measurement device (21-23). As described in Chapter 4, we evaluated the analytical performance of two POC lactate testing devices, Lactate Pro (LP) and Stastrip Lactate (SSL) and compared them to Rapidlab 860 (RL), which served as a reference method. We found that both SSL and LP can be used as a POC device for lactate measurements in FBS. SSL showed the smallest coefficient of variance, the closest correlation to and the highest agreement with RL. With the use of the regression equation generated with 38 FBS samples from this study, we converted the RL lactate cut-off values 5.4 and 6.0 to SSL (5,7 and 7.0). In the laboratory setting SSL showed the best characteristics. As described in Chapter 5, the value of SSL for its use in the clinical setting remained to be evaluated. For this purpose, we assessed the agreement of SSL, lactate (RLL) and $\mathrm{pH}(\mathrm{RLpH})$ measured on the RapidLab blood gas analyzer. We also assessed the discrepancy rates between the methods using the previously established SSL cut-off values that served as indicators for both reassurance and immediate delivery. We showed that the lactate level in FBS is accurately measured by SSL with a much lower failure rate compared to RLpH and RLL. SSL cut-off values provided correct information on fetal status in the vast majority of cases.

We also found a poor correlation between SSL and RLpH and that discrepancies are quite common. As both parameters might reflect different aspects of a developing acidemia, one may question whether these discrepancies should be considered false positive or false negative test results, or that $\mathrm{pH}$ is not the correct gold standard. A low $\mathrm{pH}$ with a normal lactate can be due to transient impairment of the carbon dioxide exchange following umbilical cord compression or an early stage of a fastdeveloping acidosis. A normal $\mathrm{pH}$ with a high lactate can be explained by: (1) slow clearance of lactate from the fetal circulation and fast normalization of $\mathrm{pH}$ sometime after a hypoxic insult; (2) an early stage of a slowly developing acidosis; (3) factors other than hypoxia, such as contamination of amniotic fluid or limited local perfusion as occurs with caput formation $(24,25)$. Furthermore, the fetal acidbase balance is influenced by the administration of beta- mimetic drugs, glucose, maternal hyperventilation and catecholamine concentrations $(17,26)$. As lactate and $\mathrm{pH}$ might mirror different stages of a developing asphyxia and the SSL cut-off values have not yet evaluated prospectively regarding intervention rates and clinical outcome, it might be wise to perform parallel analysis of both $\mathrm{pH}$ and lactate.

Concern has been raised that this combined sampling might increase the rate of operative delivery for fetal distress, due to an increased rate of abnormal results compared with single tests. Liljestrom et al. showed that combined sampling results in a $66 \%$ operative delivery rate vs. $95 \%$ if single testing is used ( $\mathrm{pH}$ or lactate) 
(27). One may argue that the use of a test with a $20 \%$ failure rate is not justified. As described in Chapter 5, we identified 14\% and 5\% discrepancies between SSL and $\mathrm{RLpH}$ for the cut-off values for reassuring fetal status and immediate intervention respectively. Because of these discrepancies and to reduce the chance of missing fetal hypoxia, we propose combined testing of SSL and RLpH. To avoid repeated sampling when $\mathrm{pH}$ analysis fails, clinical management in those cases is based on SSL only.

\section{STAN and FBS}

Currently, there is intense debate as to whether STAN has a place in contemporary obstetric practice, or whether its use should be discouraged and discontinued. The role of FBS in combination with STAN is also subject of discussion. Some find it counterproductive that FBS is used to confirm or supplement the results obtained by STAN. Others do not believe in STAN and advocate continued use of CTG and FBS.

The first randomized controlled trials (RCTs) conducted in Europe showed a great promise with regard to STAN in reducing operative delivery rates and neonatal metabolic acidosis $(28,29)$, whereas subsequent studies have produced conflicting results with regarding to these outcomes $(30,31)$. The largest RCT on STAN in the United States in 2015 has concluded that the use of STAN neither reduced operative deliveries nor improved neonatal outcome (32). A subsequent metaanalysis which included six RCTs including the US trial showed that ST analysis was not associated with a reduction in operative deliveries for fetal distress, but did show a 40\% reduction in FBS usage and a 36\% reduction in the rate of neonatal metabolic acidosis. However, the authors question the clinical significance of the observed reduction in metabolic acidosis and point out that no effect on the most meaningful outcomes, including cesarean delivery, severe acidemia at birth, or encephalopathy. They conclude that there is not enough evidence to justify the use of STAN in contemporary obstetrics $(33,34)$. Other opposers of STAN argue that it is a non-intuitive method which occupies a lot of intellectual capacity (35). This may lead to the focus on the screen and the complex algorithm, instead of the focus on proactive support and the progress of labor.

After the publications of the US trial, the debate regarding the efficacy of STAN has intensified. Advocates of STAN argue that the results of the US trial may not be representative for European clinical practice, as there were a number of fundamental differences between the US trial and the European trials. In the US trial, a three tier instead of a four-tier cardiotocography (CTG) classification and ST-action system 
was used. FBS use was absent, and the recruitment rate was very low. On average, there were nine inclusions per institution per month, in contrast to $15-135$ in the five European trials. The low inclusion rate of only two per week in large units with many obstetric care givers may have hampered adequate exposure to the new monitoring technique. Data from the European trials shows that the results in favor of ST analysis were more pronounced in the second half of the trials, which suggests a continuing learning process (36). Moreover, longitudinal data from several hospitals shows that after the introduction of STAN, metabolic acidosis at birth fell over the course of several years by $50-75 \%$, without an increase in instrumental deliveries (37). The latter data suggest that an introduction of ST analysis in the labor ward may have resulted in a considerably better outcome than expected on the basis of the trials. An explanation for this could be that the introduction of the new device may have resulted in more attention towards CTG interpretation, as structured CTG classification and training are an essential part of the ST package. The better than expected outcome in the conventional arm of the Dutch RCT may also point towards an improved CTG interpretation (i.e. the 'Hawthorne effect'). Moreover, the automatic detection and depiction of (significant) ST changes forces the clinician to interpret the CTG pattern and ST events to determine clinical management. In other words, in case of possible deterioration of the fetal condition the clinician is obliged to make a documented decision. Analysis of cases with neonatal encephalopathy despite the use of STAN monitoring, revealed that human error was responsible for 13 out of 14 cases. These included: error in CTG interpretation, failures in considering the wider clinical picture such as the presence of meconium, chorioamnionitis, failure to progress in labor as well as injudicious use of oxytocin infusion and delays in intervention (38). It seems to be human error that blunts the effectiveness of the STAN technology.

As described in Chapter 6, the FBS-failure rate in our institution was at a level of $30 \%$. In order to reduce this unacceptably high failure rate, we introduced a new FBS sampling method and implemented the combined testing of SSL and pH in FBS. In addition, STAN was installed in all delivery rooms and our local clinical guideline concerning fetal monitoring was updated. The implementation of these expanded measures for fetal monitoring, resulted in an increase in FBS usage, a tenfold decrease in FBS failure rate and a lower rate of FBS per labor. This was associated with a higher proportion of spontaneous vaginal deliveries with comparable perinatal outcome.

FBS as an adjunct to STAN is somewhat controversial and some argue that FBS should be abandoned entirely (2). However, false negative results have been reported with STAN use (39-41). In fact, the STAN clinical guidelines were modified due to 
the reports of these false negative results to include recommendations for situations in which additional fetal information is needed (42). The STAN clinical guidelines do not explicitly mention FBS as a possible means to provide this additional information, the national guideline on fetal monitoring issued by the Dutch Society of Obstetricians and Gynecologists (NVOG) does recommend FBS in those situations (43). Our updated local guideline is based on this national guideline and recommends a stricter use of FBS to make it as efficient as possible. The positive predictive value is low as the a priori risk of an acidotic fetus is low. Although there was a decrease in the number of scalp samples per labor from 2.3 to 1.6 in our study, we did not see a reduction in overall FBS usage, which underlines the need for more training and critical discussion between colleagues. We also think that there should be a greater involvement of the supervising obstetrician prior to the decision to perform FBS, as this has shown to increase the pretest probability and reduce the CS rate of for fetal distress, as well as instrumental birth and unnecessary FBS (44).

In the Netherlands FBS is performed in 3 to $15 \%$ of deliveries (45). As described in Chapter 6, we found an increase in FBS usage from 12 to $19 \%$ after the implementation of our expanded fetal monitoring protocol. This increase in FBS usage was higher than reported in the literature and implies a more liberal use of FBS, i.e. for reassurance on fetal condition before active pushing is started. It could also be explained by the convenience and high success rate of SSL compared to the cumbersome procedure to obtain a $\mathrm{pH}$ result in FBS. The threshold to perform FBS might be lower when the clinician knows that an SSL result can be easily obtained when there is CTG-suspected fetal distress. In contrast, if there is high chance of failure, as is the case with $\mathrm{pH}$ analysis, it is likely that the clinician would waive FBS altogether in those situations. Another explanation for the increase in our study could be that the residents, midwives and obstetricians on our ward were not completely familiar with the STAN technique and therefore did not dare to entirely rely on the STAN clinical guidelines. Moreover, our guideline allowed for FBS to be performed in case of significant ST events in the first stage of labor, as it has been reported that the majority $(56 \%)$ of FBS samples taken in those situations show a $\mathrm{pH} \geq 7.25$ (46). Secondly, the reduction in the number of FBS per woman suggests that the clinicians relied more on ST-analysis after a reassuring FBS-result. Therefore, the implementation of ST-analysis could explain both the increase in overall FBS usage as well as the reduction in the number of repeated FBS.

As described in Chapter 6, we managed to reduce the FBS-failure rate to 3.5\% by implementing combined testing of SSL and $\mathrm{pH}$ in FBS. Recent experience with SSL on our ward shows that the failure rate can be reduced to nil by quick analysis of a 
second blood drop. This is in accordance with the literature where failure rates of 1-3\% have been reported $(15,19,47)$. Prior to the implementation of combined testing, the FBS failure rate was 30\%, which is much higher than the $10-20 \%$ reported in the literature $(15,17,48)$. This high failure rate may have resulted in large sampling to result intervals and therefore in a delay in clinical management as Tufnell and coworkers previously reported (49). We decided to perform lactate testing before $\mathrm{pH}$ analysis, as SSL delivers a result in 13 seconds. If the value is abnormal, arrangements for delivery can be made while waiting for the $\mathrm{pH}$ result, thereby keeping a potential delay in management as low as possible. In our opinion, the CTG abnormalities indicating FBS should be severe enough to warrant immediate delivery. This means that if there is no result, immediate delivery should be performed. This could mean that a high FBS failure rate might also result in unnecessary operative intervention. As described in Chapter 6, we obtained a result with the use of SSL in $86 \%$ of cases where $\mathrm{pH}$ analysis failed. Assuming that in those cases operative delivery would have been performed because of no $\mathrm{pH}$ result, one might argue that the availability of an SSL has prevented operative delivery in those cases.

The goal of intra partum fetal monitoring is to prevent adverse neonatal outcome. The low prevalence of poor neonatal outcome is a common problem in evaluating tests for fetal well-being during labor. FBS is an important tool to exclude acidosis when the CTG is abnormal or, in the case of STAN monitoring, if the physician is uncertain of fetal well-being and additional information is required. As described in Chapter 6, the implementation of STAN in our institution did not result in an improvement in neonatal outcome. Observational studies from Sweden and Norway illustrated that with increasing STAN use over time, a low neonatal metabolic acidosis rate can be achieved $(50,51)$. In these countries, STAN was introduced many years earlier. As with all new medical technologies requiring user input, a significant learning curve exists (52). Since clinical success may be highly dependent on the skill of the clinician and observed effectiveness of the technology may improve as skills develop. Furthermore, the incidence of adverse events may decrease, improving the overall benefit risk relationship of the technique. The success of both FBS and STAN depends on correct interpretation of the CTG pattern and the appropriate intervention (or non-intervention), which makes them susceptible to human fallibility. Therefore, adequate organization with continuous monitoring, teaching and learning is needed. 


\section{Implications for practice}

In conclusion, the implications for practice include:

- FBS is very useful in clinical practice through excluding metabolic acidosis, thereby compensating the high false positive rate of an abnormal CTG.

- The use of Point of Care lactate can solve the high failure rate associated with FBS.

- Discrepancies between lactate and $\mathrm{pH}$ in FBS are common. As both parameters might reflect different aspects of a developing acidemia, combined testing is recommended.

- FBS can reduce the overall rate of assisted vaginal delivery and has the potential to reduce the CS rate.

- $\quad$ STAN is useful in clinical practice through providing more attention towards CTG interpretation, classification, as well as reducing FBS usage and metabolic acidosis at birth.

- A continuous focus on the quality of intrapartum surveillance, by prioritizing the 24/7 presence of experienced clinicians on the labor ward, as well as critical case discussions and continuous training is essential for this. 


\section{Topics for future research}

On the basis of this thesis, the following topics for further research are suggested:

- A randomized trial comparing CTG to CTG with FBS in order to determine if FBS is (cost) effective in preventing CS in case of suspected fetal distress during the first stage of labor and to what extent FBS affects perinatal outcome.

- A randomized trial comparing single testing $(\mathrm{pH})$ to combined testing ( $\mathrm{pH}$ and lactate) in FBS in order to determine which method is superior in preventing operative delivery and poor neonatal outcome.

- A prospective observational cohort study comparing our fetal monitoring protocol with those in other hospitals in order to investigate the value of FBS in addition to CTG and STAN. This may be a good alternative to the above mentioned RCT's as one may question the feasibility and usefulness of randomized trials in complex settings as intrapartum fetal monitoring.

- Development of improved techniques for fetal monitoring, such as computerized analysis of fetal heart rate variability, automated interpretation of the CTG, and non-invasive analysis of the fetal ECG. 


\section{References}

(1) Westerhuis ME, van Horen E, Kwee A, van der Tweel I, Visser GH, Moons KG. Interand intra-observer agreement of intrapartum ST analysis of the fetal electrocardiogram in women monitored by STAN. BJOG. 2009;116(4):545-51.

(2) Chandraharan E, Wiberg N. Fetal scalp blood sampling during labor: an appraisal of the physiological basis and scientific evidence. Acta Obstet Gynecol Scand. 2014;93(6):544-7.

(3) Chandraharan E. Fetal scalp blood sampling should be abandoned: FOR: FBS does not fulfil the principle of first do no harm. BJOG. 2016;123(11):1770.

(4) Schaap TP, Moormann KA, Becker JH, Westerhuis ME, Evers A, Brouwers HA, et al. Cerebrospinal fluid leakage, an uncommon complication of fetal blood sampling: a case report and review of the literature. Obstet Gynecol Surv. 2011;66(1):42-6.

(5) Jorgensen JS, Weber T. Fetal scalp blood sampling in labor--a review. Acta Obstet Gynecol Scand. 2014;93(6):548-55.

(6) Low JA, Panagiotopoulos C, Derrick EJ. Newborn complications after intrapartum asphyxia with metabolic acidosis in the term fetus. Am J Obstet Gynecol. 1994;170(4):1081-7.

(7) Haverkamp AD, Orleans M, Langendoerfer S, McFee J, Murphy J, Thompson HE. A controlled trial of the differential effects of intrapartum fetal monitoring. Am J Obstet Gynecol. 1979;134(4):399-412.

(8) Alfirevic Z, Devane D, Gyte GM. Continuous cardiotocography (CTG) as a form of electronic fetal monitoring (EFM) for fetal assessment during labour. Cochrane Database Syst Rev. 2013(5):CD006066.

(9) Alfirevic Z, Devane D, Gyte GM. Continuous cardiotocography (CTG) as a form of electronic fetal monitoring (EFM) for fetal assessment during labour. Cochrane Database Syst Rev. 2006(3):CD006066.

(10) Saling E. [A new method for examination of the child during labor. Introduction, technic and principles]. Arch Gynakol. 1962;197:108-22.

(11) Goldaber KG, Gilstrap LC, 3rd, Leveno KJ, Dax JS, McIntire DD. Pathologic fetal acidemia. Obstet Gynecol. 1991;78(6):1103-7.

(12) Sehdev HM, Stamilio DM, Macones GA, Graham E, Morgan MA. Predictive factors for neonatal morbidity in neonates with an umbilical arterial cord $\mathrm{pH}$ less than 7.00. Am J Obstet Gynecol. 1997;177(5):1030-4.

(13) Low JA, Lindsay BG, Derrick EJ. Threshold of metabolic acidosis associated with newborn complications. Am J Obstet Gynecol. 1997;177(6):1391-4.

(14) Siggaard-Andersen O. An acid-base chart for arterial blood with normal and pathophysiological reference areas. Scand J Clin Lab Invest. 1971;27(3):239-45.

(15) Westgren M, Kruger K, Ek S, Grunevald C, Kublickas M, Naka K, et al. Lactate compared with $\mathrm{pH}$ analysis at fetal scalp blood sampling: a prospective randomised study. Br J Obstet Gynaecol. 1998;105(1):29-33. 
(16) Roemer VM. [Measured quantities in perinatal medicine--the base excess]. Z Geburtshilfe Neonatol. 2005;209(3):81-9.

(17) Nordstrom L, Ingemarsson I, Westgren M. Fetal monitoring with lactate. Baillieres Clin Obstet Gynaecol. 1996;10(2):225-42.

(18) Kastendieck E, Paulick R, Martius J. Lactate in fetal tissue during hypoxia; correlation to lactate, $\mathrm{pH}$ and base deficit in the fetal blood. Eur J Obstet Gynecol Reprod Biol. 1988;29(1):61-71.

(19) Wiberg-Itzel E, Lipponer C, Norman M, Herbst A, Prebensen D, Hansson A, et al. Determination of $\mathrm{pH}$ or lactate in fetal scalp blood in management of intrapartum fetal distress: randomised controlled multicentre trial. BMJ. 2008;336(7656):1284-7.

(20) East CE, Leader LR, Sheehan P, Henshall NE, Colditz PB. Intrapartum fetal scalp lactate sampling for fetal assessment in the presence of a non-reassuring fetal heart rate trace. Cochrane Database Syst Rev. 2010(3):CD006174.

(21) Kruger K, Hallberg B, Blennow M, Kublickas M, Westgren M. Predictive value of fetal scalp blood lactate concentration and $\mathrm{pH}$ as markers of neurologic disability. Am J Obstet Gynecol. 1999;181(5 Pt 1):1072-8.

(22) Allen RM, Bowling FG, Oats JJ. Determining the fetal scalp lactate level that indicates the need for intervention in labour. Aust N Z J Obstet Gynaecol. 2004;44(6):549-52.

(23) Birgisdottir BT, Holzmann M, Varli IH, Graner S, Saltvedt S, Nordstrom L. Reference values for Lactate Pro 2 in fetal blood sampling during labor: a cross-sectional study. J Perinat Med. 2017;45(3):321-5.

(24) Ingemarsson I, Arulkumaran S. Fetal acid-base balance in low-risk patients in labor. Am J Obstet Gynecol. 1986;155(1):66-9.

(25) Engidawork E, Chen Y, Dell'Anna E, Goiny M, Lubec G, Ungerstedt U, et al. Effect of perinatal asphyxia on systemic and intracerebral $\mathrm{pH}$ and glycolysis metabolism in the rat. Exp Neurol. 1997;145(2 Pt 1):390-6.

(26) Schmidt S, Langner K, Dudenhausen JW, Saling E. Measurement of transcutaneous pCO2 and pO2 in the fetus during labor. Arch Gynecol. 1985;236(3):145-51.

(27) Liljestrom L, Wikstrom AK, Hanson U, Akerud H, Jonsson M. Evaluation of the discrepancy between $\mathrm{pH}$ and lactate in combined fetal scalp blood sampling. Acta Obstet Gynecol Scand. 2011;90(10):1088-93.

(28) Westgate J, Harris M, Curnow JS, Greene KR. Randomised trial of cardiotocography alone or with ST waveform analysis for intrapartum monitoring. Lancet. 1992;340(8813):194-8.

(29) Amer-Wahlin I, Hellsten C, Noren H, Hagberg H, Herbst A, Kjellmer I, et al. Cardiotocography only versus cardiotocography plus ST analysis of fetal electrocardiogram for intrapartum fetal monitoring: a Swedish randomised controlled trial. Lancet. 2001;358(9281):534-8.

(30) Vayssiere C, David E, Meyer N, Haberstich R, Sebahoun V, Roth E, et al. A French randomized controlled trial of ST-segment analysis in a population with abnormal cardiotocograms during labor. Am J Obstet Gynecol. 2007;197(3):299 e1-6. 
(31) Ojala K, Vaarasmaki M, Makikallio K, Valkama M, Tekay A. A comparison of intrapartum automated fetal electrocardiography and conventional cardiotocography-a randomised controlled study. BJOG. 2006;113(4):419-23.

(32) Belfort MA, Saade GR, Thom E, Blackwell SC, Reddy UM, Thorp JM, Jr., et al. A Randomized Trial of Intrapartum Fetal ECG ST-Segment Analysis. N Engl J Med. 2015;373(7):632-41.

(33) Blix E, Brurberg KG, Reierth E, Reinar LM, Oian P. ST waveform analysis versus cardiotocography alone for intrapartum fetal monitoring: a systematic review and meta-analysis of randomized trials. Acta Obstet Gynecol Scand. 2016;95(1):16-27.

(34) Oian P, Blix E. Scarce scientific evidence for the use of cardiotocography plus fetal ECG ST interval analysis (STAN). Acta Obstet Gynecol Scand. 2014;93(6):570.

(35) Steer PJ, Hvidman LE. Scientific and clinical evidence for the use of fetal ECG ST segment analysis (STAN). Acta Obstet Gynecol Scand. 2014;93(6):533-8.

(36) Schuit E, Amer-Wahlin I, Ojala K, Vayssiere C, Westerhuis ME, Marsal K, et al. Effectiveness of electronic fetal monitoring with additional ST analysis in vertex singleton pregnancies at $>36$ weeks of gestation: an individual participant data metaanalysis. Am J Obstet Gynecol. 2013;208(3):187 e1- e13.

(37) Visser GH, Kessler J. It is time to introduce ST analysis for fetal monitoring in the labor ward? Acta Obstet Gynecol Scand. 2014;93(6):539-43.

(38) Coroyannakis C, Chandraharan E. Impact of 'Human Worm' on serious adverse incidents in obstetric practice. Int J Gynecol Obstet 2012;119:S312.

(39) Westerhuis ME, Kwee A, van Ginkel AA, Drogtrop AP, Gyselaers WJ, Visser GH. Limitations of ST analysis in clinical practice: three cases of intrapartum metabolic acidosis. BJOG. 2007;114(10):1194-201.

(40) Ingemarsson I, Westgren M. ST analysis. BJOG. 2007;114(11):1445.

(41) Doria V, Papageorghiou AT, Gustafsson A, Ugwumadu A, Farrer K, Arulkumaran S. Review of the first 1502 cases of ECG-ST waveform analysis during labour in a teaching hospital. BJOG. 2007;114(10):1202-7.

(42) Amer-Wahlin I, Arulkumaran S, Hagberg H, Marsal K, Visser GH. Fetal electrocardiogram: ST waveform analysis in intrapartum surveillance. BJOG. 2007;114(10):1191-3.

(43) Nederlandse Vereniging voor Obstetrie en Gynaecologie. Richtlijn Foetale Bewaking [Fetal monitoring during labor]. Available from: www.nvog.nl; June 2017 [Dutch].

(44) Lowe B, Beckmann M. Involving the consultant before fetal blood sampling. Aust N Z J Obstet Gynaecol. 2016;56(4):387-90.

(45) Westerhuis ME, Strasser SM, Moons KG, Mol BW, Visser GH, Kwee A. [Intrapartum foetal monitoring: from stethoscope to ST analysis of the ECG]. Ned Tijdschr Geneeskd. 2009;153:B259. 
(46) Becker JH, Westerhuis ME, Sterrenburg K, van den Akker ES, van Beek E, Bolte AC, et al. Fetal blood sampling in addition to intrapartum ST-analysis of the fetal electrocardiogram: evaluation of the recommendations in the Dutch STAN(R) trial. BJOG. 2011;118(10):1239-46.

(47) Ramanah R, Martin A, Clement MC, Maillet R, Riethmuller D. Fetal scalp lactate microsampling for non-reassuring fetal status during labor: a prospective observational study. Fetal Diagn Ther. 2010;27(1):14-9.

(48) Nordstrom L, Persson B, Shimojo N, Westgren M. Fetal scalp and umbilical artery blood lactate measured with a new test strip method. Br J Obstet Gynaecol. 1992;99(4):3079 .

(49) Tuffnell D, Haw WL, Wilkinson K. How long does a fetal scalp blood sample take? BJOG. 2006;113(3):332-4.

(50) Kessler J, Moster D, Albrechtsen S. Intrapartum monitoring of high-risk deliveries with ST analysis of the fetal electrocardiogram: an observational study of 6010 deliveries. Acta Obstet Gynecol Scand. 2013;92(1):75-84.

(51) Noren H, Carlsson A. Reduced prevalence of metabolic acidosis at birth: an analysis of established STAN usage in the total population of deliveries in a Swedish district hospital. Am J Obstet Gynecol. 2010;202(6):546 e1-7.

(52) Amer-Wahlin I, Kwee A. Combined cardiotocographic and ST event analysis: A review. Best Pract Res Clin Obstet Gynaecol. 2016;30:48-61. 



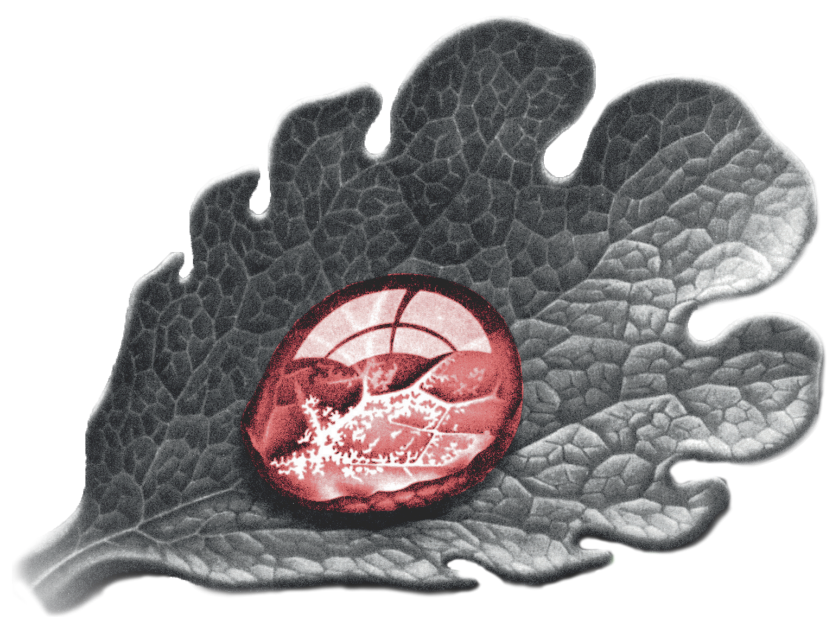




\section{Chapter 8}

SUMMARY \& SAMENVATTING 



\section{Summary}

Cardiotocography (CTG) - a simultaneous recording of the fetal heart rate and the contractions of the uterus - was introduced into obstetric practice during the $1970 \mathrm{~s}$ and 1980s and is still a key component of fetal monitoring in modern obstetrics. In Chapter 1, a general introduction on this thesis is provided. It is explained that Intra-partum CTG is a useful screening test for hypoxia and subsequent acidosis, but requires supplementary tests to avoid unnecessary operative delivery. Fetal blood sampling (FBS) and ST-analysis of the fetal electrocardiogram (STAN) are two of the best known and accepted ones. FBS with $\mathrm{pH}$ or full blood gas analysis is used to identify those fetuses with suspected distress as actually being acidotic. FBS is invasive and associated with a high failure rate which can be solved by using lactate. Point of care (POC) lactate testing devices require only a tiny amount of blood and deliver fast results with a minimal failure rate. However, lactate measurements are not standardized and values vary with each device used.

STAN is less invasive than FBS. Meta-analysis comparing STAN to CTG with or without FBS have confirmed a lower rate of metabolic acidosis, a reduction in the rate of FBS and a reduction in the overall rate of assisted vaginal delivery. There was no reduction in the rate of operative delivery for fetal distress, perinatal death, seizures and encephalopathy. It is unclear if STAN makes FBS redundant or not.

In the Netherlands, a large practice variation exists in fetal surveillance during labor. Although all Dutch labor wards use CTG, in 23\% of the hospitals, STAN is used to monitor fetal condition. In all but one hospital (98\%), FBS facilities are available, but the frequency of FBS use varies between 3-15\% of deliveries. It is suggested that this is mainly due to the variation in the indication to perform FBS. Evidence based guidelines on when to perform FBS are lacking. Furthermore, there is ongoing discussion on whether to use $\mathrm{pH}$ or lactate as a marker in FBS and there is uncertainty about the scalp lactate level at which to initiate intervention during labor. In this thesis, we have elaborated further on these challenges with the aim to guide best practice.

The quantification of the inter-and intra-observer agreement of non-reassuring intrapartum CTG patterns prior to FBS is presented in Chapter 2. Inter- and intraobserver agreement was assessed by calculating weighted kappa values $\left(\mathrm{K}_{\mathrm{w}}\right)$ and proportions of agreement $\left(\mathrm{P}_{\mathrm{a}}\right)$. Nine observers assessed CTG patterns, which were formerly clinically classified as non-reassuring and indicative for FBS, according to the CTG guidelines of the International Federation of Gynecology and Obstetrics 
modified for ST-analysis. The observers also proposed clinical management strategies with and without insight into clinical parameters. Inter-observer agreement on CTG classification and on clinical management were poor for most observer categories. Observers agreed best on abnormal CTG patterns ( $\mathrm{P}_{\mathrm{a}}$ range $0.28-0.36$ ) and on the clinical management option 'continue monitoring' ( $\mathrm{P}_{\mathrm{a}}$ range $0.32-0.40$ ). Intraobserver agreement was fair to good for most observers $\left(\mathrm{K}_{\mathrm{w}} 0.33-0.70\right)$. Insight into clinical parameters resulted in similar inter- and intra-observer agreement. From this study, it was concluded that the inter observer agreement on CTG patterns prior to FBS is poor and thus a relatively high chance that clinicians would disagree on the need to perform FBS. To ensure that FBS is as efficient as possible, the indication should be more strictly described in a well implemented guideline providing a clear association between the different CTG classifications and subsequent management.

In Chapter 3, it is described to what extent the fetal scalp blood lactate concentration during labor correlates with fetal scalp pH and base deficit, and metabolic acidosis at birth. In addition, lactate cut-off values to serve as indicators for either reassurance or immediate intervention are suggested. For this purpose, fetal scalp- and cord blood samples with acid-base and lactate values from 486 singleton pregnancies beyond 34 weeks' gestation were analyzed. Measurements were performed on a blood gas analyzer (RapidLab-860, Siemens Health Care Diagnostics). The relation between lactate, $\mathrm{pH}$ and base deficit (BD) in fetal scalp blood was tested by Spearman's rho correlation coefficient. Lactate cut-off values indicating either reassuring fetal status or immediate intervention were estimated using percentile distribution and compared to $\mathrm{pH}$ and $\mathrm{BD}$. Metabolic acidosis was defined as umbilical cord artery $\mathrm{pH}$ below 7.05 and base deficit (BD) calculated for the blood compartment above 12 $\mathrm{mmol} / \mathrm{l}$. It was found that the fetal lactate values increased with evolving metabolic acidosis. The lactate concentration correlated with both $\mathrm{pH}(\mathrm{r}=-0.50, \mathrm{p}<0.01)$ and $\mathrm{BD}(\mathrm{r}=0.48, \mathrm{p}<0.01)$. A lactate value $<5.4 \mathrm{mmol} / \mathrm{l}$ indicated reassuring fetal status, whereas a lactate $\geq 6.6 \mathrm{mmol} / \mathrm{l}$ indicated metabolic acidosis. Fetal lactate correlated better with either the absence or presence of metabolic acidosis at birth than did fetal $\mathrm{pH}$ and $\mathrm{BD}$. It was concluded that in case of a non-reassuring CTG, the fetal scalp blood lactate value provided more accurate information on fetal acid-base status than $\mathrm{pH}$ and/or BD. As lactate analysis is easier, faster, and more often successful, fetal scalp blood lactate is an attractive alternative to full blood gas analysis in FBS.

The lactate cut-off values of $5,4 \mathrm{mmol} / \mathrm{L}$ and $6.6 \mathrm{mmol} / \mathrm{l}$ are absolute values and only valid with the Rapidlab-860 (RL), which is a large blood gas analyzer requiring a minimum sampling volume of 50 microliters. The requirement of at least 50 microliters for blood gas analyzers results in high FBS failure rates. POC lactate 
testing devices are attractive for their use in FBS because of the low sampling volume $(0.7-5.0 / \mathrm{L})$, rapid results $(13-60 \mathrm{~s})$ and low failure rates $(1 \%-3 \%)$. However, due to lack of standardization, test characteristics and lactate cut-off values in FBS have to be determined for each measurement device. In Chapter 4, the evaluation of the performance of two POC lactate meters for intrapartum use is presented. The StatStrip Lactate (Nova Biomedical) and Lactate Pro (Arkray) were evaluated. To get an overall impression of performance of the StatStrip Lactate (SSL and Lactate Pro (LP), lactate concentrations were determined at three different levels according to the CLSI EP10 protocol. Total coefficients of variation (CV's) were determined based on within-day and between-day variation. Analytical performance was evaluated further using cord artery and fetal scalp blood in a comparison study. Both POC meters were compared with our lactate reference method (RL) using fetal scalp and neonatal cord blood. Deming regression analysis was performed. The CV's for SSL were lower than for LP. Consecutive lactate measurements in 37 fetal scalp and 122 cord blood samples revealed different test characteristics for the two POC devices, with SSL showing the closest correlation to RL. From this study, it was concluded that in comparison to LP, SSL showed the better analytical performance characteristics, with the lowest coefficients of variance, the closest correlation to and the highest agreement with our reference method.

We therefore chose to evaluate Statstrip lactate (SSL) for its use in FBS in the clinical setting in comparison to lactate (RLL) and $\mathrm{pH}(\mathrm{RLpH})$ by RapidLab. The outcome of this evaluation is presented in Chapter 5. For the purpose of this study we considered a SSL value $\leq 5.7 \mathrm{mmol} / 1$ indicative for reassuring fetal status and a SSL value $\geq 7.0 \mathrm{mmol} / \mathrm{l}$ representing acidemia. These cut-off values were established by introducing the RL lactate cut-off values $(5.4$ and $6.6 \mathrm{mmol} / \mathrm{L})$ into the formula: $\mathrm{SSL}=1.13^{*} \mathrm{RL}-0.39$, which was generated from the findings described in Chapter 4. The discrepancy rates were studied using cross-tabulation. We obtained 323 FBSsamples from 139 women. Parallel sampling of SSL and RLL/RLpH was performed in 247 samples. Outcome measures were the agreement and discrepancy rates between SSL, RLL and RLpH and the failure rate of all three methods. We constructed a Bland-Altman graph to assess the variability between the measurements across the range of values. SSL showed excellent agreement with RLL and poor agreement with RLpH. Failure rates for SSL, RLL and RLpH were 7\%, 43\% and 23\% respectively. Using the cut-off values for reassurance and immediate delivery, the discrepancy rates between SSL and RLpH were 14\% and 5\% respectively. It was shown that with the use of the experimentally established cut-off values, SSL provides accurate information on fetal status in the vast majority of cases. As discrepancies between 
lactate and $\mathrm{pH}$ are common and the cut-off values have not yet been evaluated and prospectively tested regarding intervention rates and neonatal outcome, we proposed combined testing of lactate (SSL) and $\mathrm{pH}$ in FBS.

To improve fetal monitoring in the Radboud University Medical Center in general and specially to reduce the high FBS failure rate of $20-30 \%$ we implemented two new procedures on our labor ward: STAN and POC lactate testing in FBS in parallel with $\mathrm{pH}$ according to a new clinical guideline on intrapartum fetal monitoring. In Chapter 6, the results of the two-year follow-up of these measures are presented. For this study, we compared to birth cohorts with singleton vertex deliveries $\geq 36$ weeks. Cohort one ranged from 2010-2011, cohort two from 2014-2016. We assessed maternal and neonatal data including mode of delivery, birthweight, Apgarscore, FBS and cord blood acid-base values. Main outcome measures were FBS failure rate, operative deliver rates and metabolic acidosis at birth. The FBS failure rate decreased from $23 \%$ to $2 \%$. The percentage of labors in which FBS was used remained stable at $12 \%$, while the number of samples per labor decreased from 2.3 to 1.6. In cases where FBS was performed, we found a significant decrease in ventouse delivery (from 37 to $27 \%$ ) and a significant increase in spontaneous vaginal delivery (from 37 to 49\%). The number of infants with low Apgar score and neonatal metabolic acidosis remained stable. From this study, it was concluded that the implementation of these two measures resulted in a tenfold decrease in FBS failure rate, fewer scalp samples per labor and a higher proportion of spontaneous vaginal deliveries with comparable perinatal outcome.

The ideal method for fetal monitoring would be simple, noninvasive, and applicable for all women in labor, irrespective of gestational age. In Chapter 7, it is explained why neither FBS, nor STAN fulfill all of these demands but that their integrated use could be the way to move forward. From the findings in this thesis it is concluded that FBS is very useful in clinical practice through excluding metabolic acidosis, thereby compensating the high false positive rate of an abnormal CTG. The high failure rate associated with FBS can be solved with the use of Point of care lactate. Discrepancies between lactate and $\mathrm{pH}$ in FBS are common and as both parameters might reflect different aspects of a developing acidemia, combined testing is recommended. FBS can reduce the overall rate of assisted vaginal delivery and has the potential to reduce the cesarean section rate. STAN is useful in clinical practice through providing more attention towards CTG interpretation, classification and, in general, a reduction in FBS usage and neonatal metabolic acidosis. A continuous focus on the quality of intrapartum surveillance, by prioritizing the $24 / 7$ presence of experienced clinicians on the labor ward, as well as critical case discussions and continuous training is 
essential for this. Currently, there is intense debate as to whether both STAN and FBS have a place in contemporary obstetric practice, or whether their use should be discouraged and discontinued. The results of our studies can help to improve fetal monitoring in daily practice. Future studies regarding the efficacy of FBS and the development of improved (non-invasive) techniques of fetal monitoring are needed. 


\section{Samenvatting}

Cardiotocografie (CTG) - een simultane registratie van het foetaal hartritme en de uteruscontracties- werd in de jaren '70 en ' 80 van de vorige eeuw geïntroduceerd in de klinische verloskunde praktijk en is nog steeds een de belangrijkste methoden voor foetale bewaking. Hoofdstuk 1 van dit proefschrift is een algemene introductie waarin wordt uitgelegd dat het intrapartum CTG weliswaar een goede screeningstest voor foetale hypoxie en daaropvolgende acidose is, maar dat aanvullende technieken nodig zijn om onnodige interventie tijdens de baring te voorkomen. Microbloedonderzoek (MBO) en ST-analyse van het foetale electrocardiogram (STAN) zijn de meest bekende en geaccepteerde technieken. Bij MBO wordt wat bloed uit de foetale (hoofd)huid afgenomen om hierin een $\mathrm{pH}$ of volledige bloedgasanalyse te verrichten. De techniek wordt gebruikt om bij afwijkende CTG-patronen na te gaan of de foetus in kwestie daadwerkelijk aan het verzuren is. MBO is invasief en geassocieerd met een grote mislukkingskans welke sterk kan worden gereduceerd door alleen lactaat te bepalen. Point of care (POC) lactaat meters hebben een zeer kleine hoeveelheid bloed nodig en leveren binnen een minuut resultaat met een minimale kans op een mislukte meting. De lactaatmeting is echter niet gestandaardiseerd en gemeten waarden variëren per apparaat.

STAN is minder invasief dan $\mathrm{MBO}$ en maakt gebruik van veranderingen in het foetale ECG die zijn geassocieerd met hypoxie van het foetale hart. Om deze veranderingen in het ECG te meten is het spiraalelectrode op het voorliggend deel noodzakelijk. Diverse meta-analyses waarin STAN is vergeleken met CTG (met of zonder MBO) lieten een reductie zien in het aantal gevallen van neonatale metabole acidose, het aantal MBO's en het aantal vaginale kunstverlossingen voor alle indicaties. Er werd geen reductie gezien in het aantal keizersneden, vaginale kunstverlossingen voor foetale nood, perinatale sterfte, convulsies en encefalopathie. Het is niet duidelijk of STAN het MBO overbodig maakt.

In Nederland bestaat er een grote praktijkvariatie in de gebruikte methoden voor intrapartum foetale bewaking. Alle verloskundeafdelingen gebruiken CTG en in 23\% van de ziekenhuizen wordt STAN gebruikt. In bijna alle ziekenhuizen zijn MBOfaciliteiten beschikbaar, maar de frequentie waarin $\mathrm{MBO}$ verricht wordt varieert van $3-15 \%$ van alle bevallingen. De variatie in indicatiestelling voor het $\mathrm{MBO}$ zou hier een oorzaak van kunnen zijn. Evidence-based richtlijnen over wanneer een $\mathrm{MBO}$ te verrichten ontbreken en er is discussie over welke parameter in het $\mathrm{MBO}$ moet 
worden gebruikt. Ook is er onduidelijkheid over welke scalplactaat waarde kan dienen als afkapwaarde voor interventie. Deze vragen zijn in dit proefschrift verder uitgewerkt met als doel een leidraad te bieden voor de beroepspraktijk.

In hoofdstuk 2 wordt de kwantificering van de inter- en intra-observer overeenstemming van CTG-patronen voorafgaand aan MBO gepresenteerd. Deze overeenstemming werd vastgesteld door middel van het berekenen van gewogen kappa waarden $\left(\mathrm{k}_{\mathrm{w}}\right)$ en proporties voor overeenstemming $\left(\mathrm{P}_{\mathrm{a}}\right)$. Negen observanten beoordeelden CTG-patronen die voorheen in de kliniek waren geclassificeerd als niet geruststellend en indicatief voor MBO. De negen observanten deden ook beleidsvoorstellen met en zonder inzicht in de klinische parameters. De inter-observer overeenstemming over de CTG-classificatie en het klinisch beleid was in de meeste categorieën slecht. De meeste overeenstemming was er in de categorie abnormaal CTG ( $P_{a}$ range $0.28-0.36$ ) en het beleidsvoorstel 'continueer CTG' $\left(\mathrm{P}_{\mathrm{a}}\right.$ range 0.32 -0.40 ). Inzicht in de klinische parameters resulteerde in een inter en intra observer overeenstemming van gelijke grootte. De conclusie van deze studie was dat de inter observer overeenstemming over CTG-patronen voorafgaand aan $\mathrm{MBO}$ slecht is en er daarmee een relatief grote kans bestaat dat clinici het niet eens zijn over de indicatie tot $\mathrm{MBO}$. Om $\mathrm{MBO}$ zo efficiënt mogelijk te maken, is het nodig de indicatiestelling nauwkeuriger te omschrijven in een goed geïmplementeerde klinische richtlijn met een duidelijke associatie tussen de verschillende CTG-klassen en de daaruit volgende beleidsopties.

In hoofdstuk 3 wordt beschreven in welke mate de scalplactaatconcentratie tijdens de baring correleert met de scalp-pH en base deficit en metabole acidose bij de geboorte. Ook worden afkapwaarden voor lactaat voorgesteld die kunnen dienen als indicatoren voor geruststelling of onmiddellijke interventie. Voor dit doel werden foetale scalp- en navelstrengbloedmonsters met zuur-base- en lactaatwaarden van 486 eenling zwangerschappen boven de 34 weken geanalyseerd. De metingen werden uitgevoerd met een bloedgasanalyse apparaat (RapidLab 860, Siemens Health Care Diagnostiscs). De relatie tussen lactaat, $\mathrm{pH}$ en base deficit (BD) in foetaal scalpbloed werd getoetst middels Spearman's rho correlatiecoëfficiënt. Afkapwaarden voor lactaat indicatief voor geruststelling dan wel onmiddellijke interventie werden geschat door middel van percentielverdeling en vergeleken met de $\mathrm{pH}$ en de $\mathrm{BD}$. Metabole acidose werd gedefinieerd als een $\mathrm{pH}$ beneden de 7.05 en een BD boven de $12 \mathrm{mmol} / \mathrm{L}$ in de navelstrengarterie. Er werd gevonden dat de scalp lactaatwaarden stegen bij een zich ontwikkelende metabole verzuring. De lactaatconcentratie correleerde zowel met de $\mathrm{pH}(\mathrm{r}=-0.50, \mathrm{p}<0.01)$ als met de BD $(\mathrm{r}=0.48, \mathrm{p}<0.01)$. Een lactaatwaarde $<5.4 \mathrm{mmol} / \mathrm{L}$ was indicatief voor een geruststellende foetale status, terwijl een 
lactaatwaarde $\geq 6.6 \mathrm{mmol} / \mathrm{L}$ metabole acidose anngaf. Scalplactaat correleerde beter met zowel de aan-als afwezigheid van metabole acidose bij de geboorte dan de scalp$\mathrm{pH}$ of $-\mathrm{BD}$. Er werd geconcludeerd dat in geval van een niet geruststellend CTG, de scalplactaatwaarde meer informatie verschaft over de foetale zuur-base status dan de $\mathrm{pH}$ en/of BD. Omdat de analyse van lactaat, gemakkelijker, sneller en vaker succesvol is, kan de lactaatbepaling een aantrekkelijk alternatief zijn voor de $\mathrm{pH} /$ bloedgasanalyse in het $\mathrm{MBO}$.

De lactaat afkapwaarden van 5,4 mmol/L en 6,6 mmol/L zijn absolute waarden en gelden alleen voor de Rapidlab-860 (RL), een groot bloedgas analyse apparaat welke minimaal 50 microliter nodig heeft voor analyse. Met name dit grote volume leidt tot een hoog mislukkingspercentage (tot 30\%)van het MBO. POC-lactaat meters hebben een heel klein volume nodig $(0.7-5.0 \mathrm{u} / \mathrm{L}$, leveren snel resultaat $(13-60 \mathrm{~s})$ met een zeer lage mislukkingspercentage (1\%-3\%). Dit maakt POC lactaatmeters voor gebruik in $\mathrm{MBO}$ erg aantrekkelijk. De POC-lactaatmeting is echter niet gestandaardiseerd, wat maakt dat testkarakteristieken en afkapwaarden voor gebruik in het $\mathrm{MBO}$ voor ieder meetinstrument moeten worden vastgesteld. In hoofdstuk 4 wordt de evaluatie van twee POC lactaatmeters voor intrapartum gebruik gepresenteerd. De StatStrip Lactate (Nova Biomedical) en de Lactate Pro (Arkray) werden geëvalueerd en vergeleken met onze lactaat referentie methode (RL). Om een globale indruk te krijgen van de prestaties van de StatStrip Lactate (SSL en de Lactate Pro (LP) werd de lactaatconcentratie op drie verschillende niveaus gemeten volgens het CLSI EP10 protocol. Coëfficiënten van variatie (CV's) warden bepaald op basis binnen en tussen daagse-variatie. De analytische prestaties van beide POC meters werden verder geëvalueerd met behulp van scalp- en navelstrengbloed monsters en vergeleken met de RL. Hiervoor werd Deming regressieanalyse uitgevoerd. De variantie coëfficiënten voor de Statstrip Lactate (SSL) waren lager dan die van de Lactate Pro (LP). Opeenvolgende metingen in 37 foetale scalp bloedmonsters toonde verschillende testkarakteristieken voor de twee POC meters. De conclusie van deze studie was dat in vergelijking met de LP, de SSL de beste analytische prestaties leverde met de laagste variantie coëfficiënten en de sterkste correlatie met de referentiemethode vertoonde.

We hebben er daarom voor gekozen om de SSL voor gebruik in het MBO te evalueren in de klinische setting in vergelijking met de lactaat (RLL) en $\mathrm{pH}(\mathrm{RLpH})$ meting door het RapidLab 860 bloedgasanalyse apparaat. De uitkomsten van dit onderzoek zijn beschreven in hoofdstuk 5. Voor deze studie zijn een SSL-waarde $\leq 5.7 \mathrm{mmol} / \mathrm{l}$ beschouwd als indicatief voor een geruststellende foetale status en een SSL-waarde $\geq 7.0 \mathrm{mmol} / \mathrm{l}$ representatief voor acidose. Deze afkapwaarden 
zijn vastgesteld door de RL-lactaatwaarden $(5.4$ and $6.6 \mathrm{mmol} / \mathrm{L}$ ) om te rekenen met de formule SSL $=1.13^{*} \mathrm{RL}-0.39$, afkomstig uit de bevindingen beschreven in hoofdstuk 4. Discrepanties tussen de verschillende metingen zijn bestudeerd middels kruistabellen. Er zijn 323 MBO-samples van 139 vrouwen verkregen. Parallelle analyse van SSL and RLL/RLpH werd uitgevoerd in 247 samples. De uitkomstmaten waren; de overeenstemming en discrepantie percentages tussen SSL, RLL and RLpH en het mislukkingspercentage van de drie methoden. Een Bland-Altman plot werd geconstrueerd om de variabiliteit van de metingen langs de range van de waarden te bepalen. De SSL toonde uitstekende overeenstemming met RLL en zeer matige overeenstemming met RLpH. De mislukkingspercentages voor SSL, RLL and RLpH waren respectievelijk 7\%, 43\% en 23\%. Gebruikmakend van de afkapwaarden voor geruststelling en onmiddellijke beëindiging van de baring waren de discrepantie percentages tussen SSL en RLpH respectievelijk $14 \%$ en $5 \%$. Gebruikmakend van de experimentele afkapwaarden, verschafte de metingen op de SSL accurate informatie over de foetale status in de overgrote meerderheid van de gevallen. Aangezien discrepanties tussen lactaat en $\mathrm{pH}$ vaak voorkomen en de afkapwaarden nog niet prospectief getoetst zijn voor wat betreft interventies en neonatale uitkomst, wordt gecombineerd testen van lactaat (SSL) en $\mathrm{pH}$ voorgesteld.

Hoofdstuk $\mathbf{6}$ beschrijft de tweejarige follow-up van een tweetal methoden die zijn geïmplementeerd op de verlosafdeling van het Radboudumc teneinde de foetale bewaking te verbeteren en met name het hoge MBO-mislukkingspercentage van $20-30 \%$ terug te dringen. De twee methoden waren; STAN en POC-lactaatmeting met de SSL in combinatie met $\mathrm{pH}$ volgens een nieuwe richtlijn foetale bewaking. Voor deze studie zijn twee geboorte cohorten van eenling partus van 36 weken of meer met elkaar vergeleken. Cohort één bestond uit de tijdsperiode 2010-2011, cohort twee omvatte de jaren 2014-2016. Maternale en neonatale data, inclusief modus partus, geboortegewicht, Apgarscore, $\mathrm{MBO}$ en navelstrengwaarden werden verzameld. Primaire uitkomstmaten waren: het $\mathrm{MBO}$-mislukkingspercentage, het aantal kunstverlossingen en het aantal gevallen van neonatale metabole acidose. Het percentage bevallingen waarin $\mathrm{MBO}$ werd verricht bleef stabiel op $12 \%$, terwijl het aantal MBO's per baring daalde van 2,3\% naar 1,6\%. Het MBOmislukkingspercentage daalde van 23\% naar 2\%. Bij de baringen waar MBO werd verricht was een significante daling te zien in het aantal kunstverlossingen (van $37 \%$ naar $27 \%$ ) en was er een significante stijging van het aantal spontane vaginale baringen (van 37\% naar 49\%). Het aantal kinderen met een lage Apgarscore en neonatale metabole acidose bleef stabiel. Vanuit de resultaten van deze studie werd 
geconcludeerd dat de implementatie van deze twee maatregelen, geresulteerd hebben in een 10-voudige afname van het percentage mislukte MBO's, minder MBO's per baring en meer spontane vaginale baringen bij een vergelijkbare perinatale uitkomst.

De ideale methode voor foetale bewaking is simpel, non-invasief en zou geschikt moeten zijn voor alle vrouwen in partu, ongeacht de zwangerschapsduur. In hoofdstuk 7, wordt uitgelegd waarom noch $\mathrm{MBO}$, noch STAN aan deze criteria voldoen, maar dat een geïntegreerd gebruik van beide methoden een manier kan zijn om de kwaliteit van foetale bewaking te verbeteren. Vanuit de bevindingen in dit proefschrift wordt geconcludeerd dat $\mathrm{MBO}$ een nuttig instrument is om metabole acidose uit te sluiten om daarmee het hoge aantal fout-positieve waarnemingen voor foetale nood van een abnormaal CTG te compenseren. Het hoge mislukkingspercentage dat met $\mathrm{MBO}$ geassocieerd is kan worden teruggedrongen door POC lactaat te gebruiken. Discrepanties tussen lactaat en $\mathrm{pH}$ in het $\mathrm{MBO}$ komen veel voor en omdat beide parameters een weerspiegeling zouden kunnen zijn van verschillende aspecten van een zich ontwikkelende acidemie wordt gecombineerd testen aanbevolen. MBO kan het overall percentage vaginale kunstverlossingen reduceren en heeft de potentie om ook het keizersnede percentage omlaag te brengen. STAN is van nut in de klinische praktijk omdat het gebruik van deze techniek leidt tot meer aandacht voor CTGinterpretatie, classificatie en over het algemeen minder MBO's en minder neonatale metabole acidose. Hiervoor is een continue focus op de kwaliteit van intrapartum bewaking door de 24/7 beschikbaarheid van ervaren clinici op de verlosafdeling en kritische casusbesprekingen essentieel. Momenteel is er intens debat over de vraag of zowel STAN als MBO een plaats hebben in de moderne verlospraktijk of dat het gebruik van deze methoden moet worden ontmoedigd en afgeschaft. De resultaten van de studies in dit proefschrift kunnen helpen foetale bewaking in de dagelijkse praktijk te verbeteren. Meer onderzoek naar de effectiviteit van MBO en de ontwikkeling van verbeterde (non-invasieve) foetale bewakingstechnieken is hierbij nodig. 



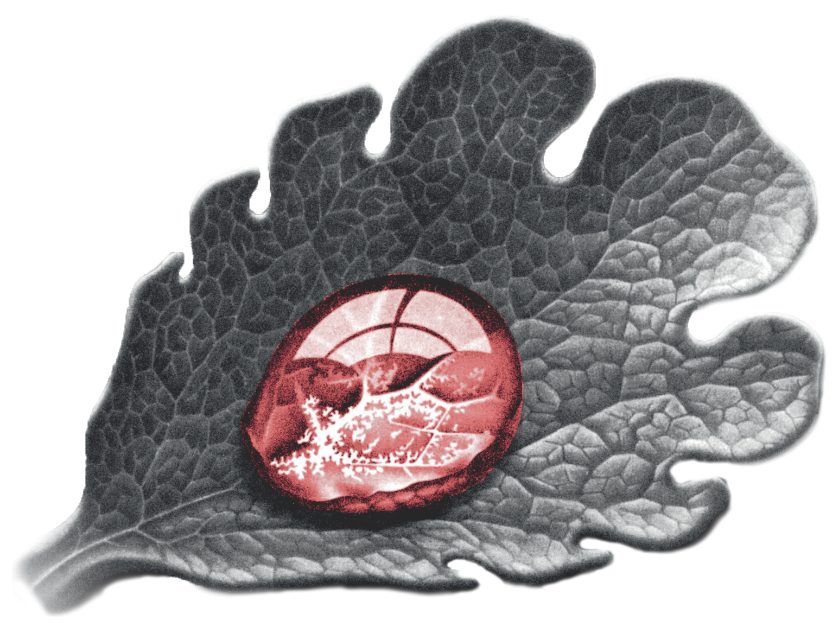




\section{Addendum}

DANKWOORD

CURRICULUM VITAE 



\section{Dankwoord}

$\mathrm{Na}$ zeven jaar is het dan eindelijk zover, het boekje is af. Graag wil ik van de gelegenheid gebruikmaken om alle mensen te bedanken die direct of indirect een bijdrage hebben geleverd aan de totstandkoming van dit proefschrift. Zonder jullie hulp was 21 maart 2018 een hele gewone werkdag was geweest!

Professor Vandenbussche, beste Frank. Dank voor de mogelijkheid die je me hebt geboden als verloskundige te kunnen promoveren. Onze samenwerking begon toen ik weliswaar een tweetal artikelen had gepubliceerd maar niet meer voornemens was om te gaan promoveren. Jij kwam met het idee om een gerandomiseerde studie op te zetten naar de effectiviteit van het $\mathrm{MBO}$. Bewijs voor die effectiviteit was immers schaars en gedateerd. De Scalp-trial is er helaas nooit gekomen, maar het werken aan de opzet van deze studie heeft mede bijgedragen aan de totstandkoming van dit proefschrift.

Doctor van Drongelen, beste Joris. Wie had gedacht dat het zo gaan toen ik jou als poortarts ging inwerken op de verloskamers van ziekenhuis Bernhoven te Veghel. Ik leerde je vliezen breken, schedelelectrodes plaatsen en episiotomieën hechten, want dat waren, zo was mij van hogerhand ingefluisterd, de belangrijkste vaardigheden die een poortarts onder de knie moest krijgen. Wat was het leuk om je later in het Radboud, als gynaecoloog in opleiding weer tegen te komen. Toen heb ik op mijn beurt veel van jou geleerd. In de patiëntenzorg, maar vooral op onderzoeksgebied. Wat een mazzel had ik dat jij ook nog epidemioloog bent. Je humor gaf me altijd weer de moed om door te gaan. Je hanteerde daarbij meer dan eens de uitspraak: "Komt goed, alleen niet vandaag".

Doctor van Dillen, beste Jeroen. Jij raakte ook pas in een later stadium betrokken bij dit hele gebeuren. Door een moeilijke tijd in mijn leven lag het onderzoek al een tijd stil. Jij hebt me gestimuleerd de draad weer op te pakken. Dankzij jouw enthousiasmerende begeleiding en bemoedigende mails zijn de laatste drie hoofstukken van dit proefschrift een feit. Bij het schrijven van de algemene discussie was er sprake van een langdurige uitdrijving, welke dankzij jouw begeleiding toch is geëindigd in een spontane bevalling.

Professor Lotgering, beste Fred. Halverwege mijn masteropleiding, kwam ik met het idee om onderzoek te doen naar lactaat in het MBO. Vrijwel direct kwam jij met een aantal publicaties op Pubmed aanzetten waar ik wat aan zou kunnen hebben. Later volgden zinvolle suggesties over hoe dit aan te pakken. In een van de eerste mails 
aan mij schreef je: 'dit is alleszins publiceerbaar en daar moet dan ook naar worden gestreefd'. Okay dacht ik, een kwestie van mijn masterthese in het Engels vertalen. Niets bleek minder waar. Wat was dat eerste artikel een kluif? $\mathrm{Na}$ de data op zo'n beetje alle mogelijke manieren bekeken te hebben, was ik soms de totale verzuring nabij toen wederom een versie rood van de feedback retour kwam. Toen ik mijn wanhoop op een bepaald moment met je deelde, stuurde je een bemoedigende mail terug die eindigde met: Volhardt! Kort daarna was het rond.

Professor Spaanderman, beste Marc. Samen met Fred heb je aan de wieg gestaan van dit proefschrift en kan ik stellen dat ik op wetenschappelijk gebied het meeste van jullie heb geleerd. Jij en Fred zaten soms niet op een lijn, wat weleens moeilijk voor mij was, maar jullie vulden elkaar ook goed aan. Jij wist bijvoorbeeld de dataset in SPSS aan de praat te krijgen en had veel ideeën over welke analyses erop los te laten. Ook met het schrijven zelf heb je me erg goed geholpen. Je zat nooit ruim in je tijd, maar als ik dan weer eens met een SPSS-uitdraai kwam aanzetten waar je wel wat in zag, noemde je dat gekscherend 'Nobel prize winning stuff' en nam je dan toch de tijd om verder te helpen.

Dit proefschrift is kritisch beoordeeld en vervolgens goedgekeurd door de leden van de manuscriptcommissie waarvoor ik hen hartelijk wil danken.

Doctor Wiberg, dear Nana. I have known your scientific work on fetal blood sampling and lactate for quite some time. Those papers helped me gain insight in the complex matter that fetal scalp blood sampling is. Maybe this is the reason why our encounter at the scientific meeting of the American Association for Clinical Chemistry (AACC) on Point of Care testing in Copenhagen was familiar somehow. You kindly invited me and Shannon to your home where we had a very pleasant stay. I hope your questions at my defense won't be too hard to answer and I'm looking forward for you and Linda to meet my family and friends at the party afterwards.

Beste Jacqueline Klein Gunnewiek en Janine Oosting, wanneer je een lactaatmeter wilt beoordelen op bruikbaarheid in het $\mathrm{MBO}$, is de kennis, kunde en het netwerk van een klinisch chemicus onontbeerlijk. Jacqueline, jij kwam destijds met het lumineuze idee om met de data van alle MBO's en navelstrenggassen uit het lab onderzoek te gaan doen, wat de basis vormde voor hoofdstuk 3 en 4 van dit proefschrift.

Beste Jacqueline Dinnissen, Gerald Dulos en Egbert Brouwer. Dankzij jullie hulp kwam de database op orde en is het klinische gedeelte van de studie op de verloskamer goed verlopen. 
Beste Liesbeth Scheepers, ook jij hebt een rol gespeeld bij het ontstaan van dit proefschrift, want ik had jou gekozen als mijn begeleider tijdens de masteropleiding. Door je vertrek naar Maastricht was dat helaas van korte duur en heeft Marc je rol overgenomen. In die korte tijd heb je toch heel wat van mijn verslagen en opdrachten van zinvolle feedback voorzien. Toen ik uiteindelijk mijn masterthese moest gaan schrijven en ik op een congres Yves Jacquemyn had horen spreken over scalplactaat, was je direct enthousiast en heb je me in contact gebracht met de juiste mensen.

Dear Andrei Malic and Germano Ferrari from Nova Biomedical. Thank you for providing the lactate meters and strips. This enabled us to conduct two of the studies in this thesis. I'm also thankful for the invitation to the AACC scientific meeting in Copenhagen almost two years ago. This gave me renewed energy to complete my research. Also, many thanks for your proof reading, Andrei. It has improved the manuscript substantially.

Lieve Sarah, via Jeroen heb ik je leren kennen toen je als student geneeskunde invulling zocht voor je wetenschapsstage. Hoe tof is het dat de dataset die wij destijds hebben aangelegd hebben, heeft geresulteerd in maar liefst drie artikelen, namelijk hoofdstuk 2, 5 en 6 van dit proefschrift. Ik vond het heel leuk om je samen met Jeroen te mogen begeleiden en ben ik dankbaar dat jij, samen met mijn broer Nils, straks als paranimf aan mijn zijde staat. En als staan niet meer gaat met die dikke buik dan ga je gewoon zitten hoor!

Lieve Marijke, dankzij jouw burgerlijke ongehoorzaamheid is het allemaal begonnen. Toen Ineke interesse toonde in de masteropleiding voor klinische verloskundigen en ik kort daarna volgde, besloot jij als leidinggevend verloskundige, ondanks dat er eigenlijk maar plek was voor één, dat wij allebei naar Rotterdam moesten gaan. Ook vond je het belangrijk dat ik tijd kreeg voor het onderzoek en heb je ervoor gezorgd dat ik daar wat tijd van de baas voor kreeg.

Lieve Ineke, samen startten wij in 2006 met de masteropleiding voor klinische verloskundigen. Twee- en-een half jaar met de trein op en neer van Nijmegen naar Rotterdam, wat een leuke tijd was dat. Ik denk niet dat ik het zo leuk had gevonden wanneer ik in mijn eentje was gegaan en denk er vaak aan terug. We waren echt maatjes en hebben elkaar veel geholpen met de overdaad aan opdrachten die er soms was. Ook zijn we samen naar Zweden gegaan voor de buitenland stage en hebben veel opgestoken van hoe de verloskundige zorg daar is georganiseerd. De presentatie die we erover maakten werd daar goed ontvangen op de werkvloer. 
Beste oud-collega verloskundigen, verpleegkundigen, gynaecologen (i.o) en alle andere collega's die ik nog ken uit mijn tijd in het Radboud. Bedankt voor de prettige samenwerking. Ook in moeilijke tijden heb ik jullie steun mogen ervaren.

Beste Loes, Marije en andere medewerkers van het secretariaat verloskunde en gynaecologie. Bedankt voor alle logistieke hulp rondom de totstandkoming van dit proefschrift. Met name ook voor de goede organisatie van het symposium voorafgaand aan mijn verdediging.

Lieve broer Nils, ik ben erg blij dat je straks samen met Sarah aan mijn zijde staat tijdens de verdediging. Wie had dat ooit gedacht toen we jong waren. We hadden vaak mot, maar konden ook veel lachen. We hebben best veel meegemaakt samen. Het overlijden van mama hakte erin. Tijdens haar ziekte heb je veel voor haar gezorgd hebt wat zwaar voor je moet zijn geweest. Je was er ook voor mij toen ik het moeilijk had. Dank daarvoor. Door alle drukte van een gezin en werk, zien we elkaar te weinig vind ik. Zullen we daar maar eens verandering in aanbrengen? Te beginnen bij vandaag.

Lieve papa, je bent nu al 47 jaar mijn vader en ik realiseer me dat ik veel van jou in me heb. Het kritische opvliegende, maar ook de doortastendheid, diepgravend- en gedrevenheid. Deze eigenschappen hebben me gebracht tot waar ik nu ben. Als klein meisje al luisterde ik altijd met veel aandacht naar de verhalen over wat je als dokter had meegemaakt. De verhalen over psychiatrie en verloskunde in het bijzonder. Toen ik mijn interesse voor de verloskunde aan jou en mama kenbaar maakte was je zichtbaar opgetogen. Je zei: "verloskunde, dat past bij jou. Het is een vak wat je uitoefent met je hoofd, je handen en je hart". Dat ben ik roerend met je eens.

Lieve Boukje, je bent nu al weer 16 jaar mijn stiefmoeder, al vind ik dat een rare term. Dank voor de liefde en zorg die je ons geeft. Nu ik wat meer tijd heb hoop ik dat we elkaar wat vaker zullen gaan zien. Misschien moeten Shannon en ik jullie binnenkort maar eens een privélesje salsadansen komen geven. Onderzoek heeft immers uitgewezen dat dansen, in vergelijking met fitness, de beste manier is om veroudering van het brein tegen te gaan ${ }^{1}$.

Beste Bas, we zijn niet meer samen maar blijven altijd ouders van vier prachtige kinderen. Ik ben je dankbaar voor de mooie tijden die we samen hebben gekend en de keren dat je de kinderen hebt opgevangen als ik boven zat te schrijven.

1. Rehfeld K. et al. Dancing or Fitness Sport? The Effects of Two Training Programs on Hippocampal Plasticity and Balance Abilities in Healthy Seniors. Front Hum Neurosci. 2017 Jun 15;11:305. 
Meint, Cees, Loet en Simon, lieve kinderen van mij. Wat ben ik blij dat jullie in mijn leven zijn en wat is het leuk om jullie te zien opgroeien. Ik ben heel trots op jullie, want ik heb gezien dat jullie goed voor mij en anderen kunnen zorgen. Nu dit alles achter de rug is heb ik gelukkig meer tijd voor jullie. Het vaak gehoorde "Zit je nu alweer achter je laptop, mama" behoort definitief tot het verleden.

Lieve Cees en Mieke, wat was het fijn dat we tijdelijk bij jullie in huis konden wonen en wat hebben jullie ons goed geholpen om zaken goed op de rit te krijgen.

Lieve Billy en Neeltje, dankzij jullie kan ik ook een beetje ervaren hoe het is om dochters te hebben. Ik vind het fijn om te zien dat jullie steeds meer vertrouwd met me raken. Ook geniet ik van jullie zangkunsten en ben ik heel blij dat jullie je vader weer aan het salsa dansen hebben gekregen.

Lieve Shannon, allerliefste man van mij. Als jij er niet was geweest dan denk ik niet dat dit proefschrift ooit was afgekomen. Je was mijn eerste hulp bij Excel problemen, paniek en/of driftaanvallen. Als ik weer eens huilende opbelde omdat het niet lukte, dan zei je altijd heel kalm: "Ik moet om je lachen, je bent zo voorspelbaar. Altijd volg je hetzelfde patroon; je gaat huilen en dan is het de volgende dag af". Je hebt steeds gelijk gekregen. Liefste, wat ik je verder nog te zeggen heb blijft tussen ons, maar wat ik hier wil uitspreken is dat ik hoop nog lang met jou door dit leven te kunnen dansen. 



\section{Curriculum Vitae}

Ayesha Maria Francisca Heinis werd op 8 maart 1971 in Amstelveen geboren. Haar moeder beviel thuis -op de studentencampus Uilenstede - onder de hoede van haar huisarts.

Als peuter gaf zij al blijk van wetenschappelijke nieuwsgierigheid. Genesteld onder het bureau van haar studerende vader bracht zij uren door met het bekijken van atlassen anatomie en dermatologie.

Haar adolescentieperiode bracht zij door in de Achterhoek, bezocht het VWO aan het Ulenhofcollege te Doetinchem. Een duidelijk beeld van haar toekomst had zij op dat moment nog niet: misschien iets in de gezondheidszorg. Op naar de HBO-V aan de Hogeschool Enschede. Positieve verhalen van haar vader over de obstetrie hielpen haar bij de keuze voor verloskunde. Aan de Vroedvrouwenschool te Kerkrade volgde zij de opleiding tot verloskundige die zij cum laude afsloot.

$\mathrm{Na}$ een aantal jaren als verloskundige in de eerste lijn te hebben gewerkt, maakte zij de switch naar de klinische verloskunde, eerst in ziekenhuis Bernoven te Veghel en vanaf 2006 in het Radboud UMC. Hier groeide haar wetenschappelijke belangstelling en zag zij het belang in van verdere professionalisering en inhoudelijke verdieping van haar beroep. Daartoe volgde zij aan de Hogeschool Rotterdam de opleiding tot Master Physician Assistant Klinisch Verloskundige, die zij cum laude afsloot. Inmiddels werkt zij als docent aan deze opleiding.

Uit haar vorige relatie heeft Ayesha vier zonen. Met haar nieuwe levenspartner beoefent zij met passie Zuid-Amerikaans dansen. Net als dansen vraagt verloskunde om een harmonieus samengaan van leiden en volgen. "It takes two to tango". 


